MAYRA BITTENCOURT VILLALPANDO

STIMULI AND FEATURE EXTRACTION METHODS FOR EEG-BASED BRAIN-MACHINE INTERFACES: A SYSTEMATIC COMPARISON 


\section{STIMULI AND FEATURE EXTRACTION METHODS FOR EEG-BASED} BRAIN-MACHINE INTERFACES: A SYSTEMATIC COMPARISON

MSc. Dissertation presented to the Polytechnic School of the University of São Paulo to obtain the degree of Master of Science. 


\title{
STIMULI AND FEATURE EXTRACTION METHODS FOR EEG-BASED BRAIN-MACHINE INTERFACES: A SYSTEMATIC COMPARISON
}

MSc. Dissertation presented to the Polytechnic School of the University of São Paulo to obtain the degree of Master of Science.

Concentration Area: Control Engineering and Mechanical Automation

\author{
Advisor: $\quad$ Prof. Dr. Arturo Forner-Cordero \\ (University of São Paulo) \\ Co-Advisor: Prof. Dr. Natalia M. Maurits \\ (University of Groningen)
}

São Paulo 
Autorizo a reprodução e divulgação total ou parcial deste trabalho, por qualquer meio convencional ou eletrônico, para fins de estudo e pesquisa, desde que citada a fonte.

I authorize the total or partial reproduction and disclosure of this work, by any conventional or electronic means, for study and research purposes, provided that the source is cited.

Villalpando, Mayra Bittencourt

Stimuli and feature extraction methods for EEG-based brain-machine interfaces: a systematic comparison / M. B. Villalpando -- São Paulo, 2017. $107 \mathrm{p}$.

Dissertação (Mestrado) - Escola Politécnica da Universidade de São Paulo. Departamento de Engenharia Mecatrônica e de Sistemas Mecânicos.

1.Interface homem-computador 2.Processamento de sinais biomédicos 3.Eletroencefalografia 4.Potenciais evocados 5.Neurociências I.Universidade de São Paulo. Escola Politécnica. Departamento de Engenharia Mecatrônica e de Sistemas Mecânicos II.t. 


\section{APPROVAL SHEET}

NAME: $\quad$ VILLALPANDO, Mayra Bittencourt

TITLE: $\quad$ Stimuli and feature extraction methods for EEG-based brain-machine interfaces: a systematic comparison

MSc. Dissertation presented to the Polytechnic School of the University of São Paulo to obtain the degree of Master of Science.

APPROVED IN:

Prof. Dr.

Institution:

Judgement:

Prof. Dr.

Institution:

Judgement:

Prof. Dr.

Institution:

Judgement: 
To my mother, for loving me unconditionally and raising me to follow my dreams. 


\section{ACKNOWLEDGEMENTS}

I would like to express my sincere gratitude:

To Prof. Arturo, for receiving me back in academy with doors wide open when I felt ready for it and I needed it the most.

To Prof. Natasha, for accepting to host my journey and shedding so much light on it.

To all participants, for dedicating their time to this research and doing that so nicely. I learned a lot from them.

To my family and friends, for supporting me in all decisions I make, regardless of where they would take me; for always being there for me wherever I go and whenever I return. Special thanks to Claudia F. Mello: without her, I would probably have never known about the opportunity of doing an internship in Groningen.

To all colleagues of the Biomechatronics Laboratory of the Polytechnic School (USP), for helping me, making me proud of our institution and giving me so much hope for the future.

To all colleagues of the University Medical Center Groningen and of the University of Groningen, for making me feel at home, so far from home.

To CNPq and Erasmus+ for the financial support.

This work would not have been possible without you and I will be always thankful. 
"It is our choices, Harry,

that show what we truly are, far more than our abilities" (Rowling 1999). 


\section{RESUMO}

A interface cérebro-máquina (ICM) é um sistema que permite a comunicação entre o sistema nervoso central e um dispositivo externo (Wolpaw et al., 2002). Aplicações de ICMs incluem o controle de próteses externa, cursores e teclados virtuais, para citar alguns. As ICMs desenvolvidas por vários grupos de pesquisa diferem em suas características (por exemplo, contínua ou discreta, síncrona ou assíncrona, graus de liberdade, outras) e, apesar de várias iniciativas voltadas para diretrizes de padronização, a comparação entre os estudos continua desafiadora (Brunner et al. 2015, Thompson et al., 2014). Aqui, utilizamos um equipamento EEG de 64 canais para adquirir dados de 19 participantes saudáveis ao longo da execução de três diferentes tarefas (SSVEP, P300 e híbrida) que permitiram quatro escolhas ao usuário e não exigiram nenhum treinamento prévio. Comparamos sistematicamente o desempenho "off-line" das três tarefas nos seguintes parâmetros: a) acurácia, b) taxa de transferência de informação, c) analfabetismo / ineficiência e d) preferências individuais. Além disso, selecionamos os melhores canais por tarefa e avaliamos a acurácia em função do número de eletrodos. Nossos resultados demonstraram que a tarefa SSVEP superou as demais em acurácia, ITR e analfabetismo/ineficiência, atingindo um ITR $^{* *}$ médio de 52,8 bits/min e um ITR ${ }^{\star *}$ máximo de 104,2 bits/min. Adicionalmente, todos os participantes alcançaram um nível de acurácia acima de $70 \%$ (limiar de analfabetismo/ineficiência) nas tarefas SSVEP e P300. Além disso, a acurácia média de todas as tarefas não se deteriorou ao se utilizar um conjunto reduzido composto apenas pelos melhores 8 eletrodos. Estes resultados são relevantes para o desenvolvimento de ICMs "online", incluindo aspectos relacionados à usabilidade, satisfação do usuário e portabilidade.

Palavras-chave: Interface cérebro-máquina (ICM), P300, SSVEP, eletroencefalografia (EEG).

** Cálculo "off-line" baseado na segmentação de dados. 


\begin{abstract}
A brain-machine interface (BMI) is a system that allows the communication between the central nervous system (CNS) and an external device (Wolpaw et al. 2002). Applications of BMls include the control of external prostheses, cursors and spellers, to name a few. The BMls developed by various research groups differ in their characteristics (e.g. continuous or discrete, synchronous or asynchronous, degrees of freedom, others) and, in spite of several initiatives towards standardization and guidelines, the cross comparison across studies remains a challenge (Brunner et al. 2015; Thompson et al. 2014). Here, we used a 64-channel EEG equipment to acquire data from 19 healthy participants during three different tasks (SSVEP, P300 and hybrid) that allowed four choices to the user and required no previous neurofeedback training. We systematically compared the offline performance of the three tasks on the following parameters: a) accuracy, b) information transfer rate, c) illiteracy/inefficiency, and d) individual preferences. Additionally, we selected the best performing channels per task and evaluated the accuracy as a function of the number of electrodes. Our results demonstrate that the SSVEP task outperforms the other tasks in accuracy, ITR and illiteracy/inefficiency, reaching an average ITR $^{\star *}$ of $52,8 \mathrm{bits} / \mathrm{min}$ and a maximum ITR $^{* *}$ of $104,2 \mathrm{bits} / \mathrm{min}$. Additionally, all participants achieved an accuracy level above 70\% (illiteracy/inefficiency threshold) in both SSVEP and P300 tasks. Furthermore, the average accuracy of all tasks did not deteriorate if a reduced set with only the 8 best performing electrodes were used. These results are relevant for the development of online BMIs, including aspects related to usability, user satisfaction and portability.
\end{abstract}

Keywords: Brain-machine interface (BMI), P300, SSVEP, electroencephalography (EEG).

${ }^{* *}$ Offline calculation, based on data segmentation. 


\section{LIST OF FIGURES}

FigURE 1 - PRINCIPLE OF A BRAIN-COMPUTER INTERFACE INCLUDING POSSIBLE APPLICATION SCENARIOS ((BRUNNER, Birbaumer, Blankertz, Guger, KüBler, Mattia, Millán, Miralles, Nijholt, Opisso, Ramsey, Salomon AND MÜLLER-PUTZ 2015), ADAPTED FROM (WOLPAW AND WOLPAW 2012)). 15

FigURE 2 - THE HCP'S MULTI-MODAL PARCELLATION, VERSION 1.0 (HCP_MMP1.0): THE 180 AREAS DELINEATED AND IDENTIFIED IN BOTH LEFT AND RIGHT HEMISPHERES ARE DISPLAYED ON INFLATED AND FLATTENED CORTICAL SURFACES. BLACK OUTLINES INDICATE AREAL BORDERS. COLOURS INDICATE THE EXTENT TO WHICH THE AREAS ARE ASSOCIATED IN THE RESTING STATE WITH AUDITORY (RED), SOMATOSENSORY (GREEN), VISUAL (BLUE), TASK POSITIVE (TOWARDS WHITE), OR TASK NEGATIVE (TOWARDS BLACK) GROUPS OF AREAS. THE LEGEND ON THE BOTTOM RIGHT ILLUSTRATES THE 3D COLOUR SPACE USED IN THE FIGURE. DATA AT HTTP://BALSA.WUSTL.EDU/WN56. (GLASSER ET AL. 2016)

FIGURE 3 - EFFECT OF VISUAL STIMULATION AT DIFFERENT FREQUENCIES ON THE AMPLITUDE OF THE SSVER. A) SSVER RECORDINGS OF ONE REPRESENTATIVE SUBJECT AT THE RIGHT OCCIPITAL LEAD (O2). B) FFT OF THIS INDIVIDUAL'S SSVERS AND GRAND AVERAGE OF 16 NORMAL SUBJECTS (DOTTED LINE). C) AVERAGE OF THE MEAN VALUES OF THE AMPLITUDE OF THE FFT FUNDAMENTAL FREQUENCY OF THE SSVER RECORDED AT THE THREE OCCIPITAL LEADS (OZ, CENTER; 01, LEFT; 02, RIGHT) AT THE DIFFERENT STIMULATION FREQUENCIES. THE AMPLITUDE OF THE OCCIPITAL SSVER, EXPRESSED IN MICROVOLTS, REACHED A MAXIMUM AT 15 HZ AND THEN FELL WITH A PLATEAU UP TO 27 HZ, DECLINING AT HIGHER FREQUENCIES (PASTOR ET AL. 2003).

FiguRE 4 - P300 BCI. A MATRIX OF POSSIBLE CHOICES IS PRESENTED ON A SCREEN AND SCALP EEG IS RECORDED OVER THE CENTROPARIETAL AREA WHILE THESE CHOICES FLASH IN SUCCESSION. ONLY THE CHOICE DESIRED BY THE USER EVOKES A LARGE P300 POTENTIAL (I.E. A POSITIVE POTENTIAL ABOUT 300 MS AFTER THE FLASH) (ADAPTED FROM (Donchin, Spencer And Wijesinghe 2000; WolpaW, Birbaumer, McFarland, PFurTscheller and Vaughan 2002))

FiguRE 5 - GENERAL REPRESENTATION OF A BMI DESIGN AND ITS STAGES: A) BRAIN STIMULATION, B) SigNAL ACQUISITION, C) FEATURE EXTRACtion AND D) FEATURE TransLation. 38

FIGURE 6 - GUI WITH FOUR VIRTUAL BUTTONS (VBS) AND THEIR ASSOCIATED FLICKERING FREQUENCIES: F1=15 (75/5) Hz, F2=12,5 (75/6) Hz, F3=10,71 (75/7) Hz AND F4=8,33 (75/9) Hz. THE VB WITH FREQUENCY F2 IS BEING INDICATED BY ITS ASSOCIATED CUE.

FIGURE 7 - SSVEP TASK: DIAGRAM PRESENTING ONE BLOCK THAT CONSISTS OF TWO PAUSES (INITIAL AND FINAL) AND ONE TRIAL (FURTHER SUBDIVIDED INTO FOUR SUBTRIALS). EACH SUBTRIAL IS ASSOCIATED WITH A VISUAL CUE IN ONE OF THE FOUR POSSIBLE LOCATIONS FOR THE USER CHOICE. 41

FiguRE 8 - DESIGN OF A 2X3 MATRIX WITH 4 POSSIBlE CHOICES (SYMBOLS) AND 2 DUMMY SYMBOLS FOR P300-BCI INTERFACE (ADAPTED FROM (HALDER ET AL. 2015)). ONE OF THE SYMBOLS IS BEING INDICATED BY ITS ASSOCIATED CUE AND FLASHED WITH THE FAMOUS FACE OF ALBERT EINSTEIN. 43

FIGURE 9 - P300 TASK DIAGRAM PRESENTING ONE BLOCK THAT CONSISTS OF TWO PAUSES (INITIAL AND FINAL) AND ONE TRIAL (FURTHER SUBDIVIDED INTO FOUR SUBTRIALS). EACH SUBTRIAL IS ASSOCIATED TO A VISUAL CUE IN ONE OF THE FOUR POSSIBLE LOCATIONS FOR THE USER CHOICE. 
FIGURE 10 - GUI WITH TWO VIRTUAL BUTTONS (VBS) AND THEIR ASSOCIATED FLICKERING FREQUENCIES: F2=12,5

(75/6) Hz, F3=10,71 (75/7) Hz 4 AND POSSIBLE CHOICES (SYMBOLS). EACH CHOICE REQUIRES THE SIMULTANEOUS MENTAL COUNTING OF HOW MANY TIMES THE FAMOUS FACE OF ALBERT EINSTEIN IS FLASHED ON IT AND THE VISUAL FOCUS ON THE CLOSEST VB. ONE OF THE SYMBOLS AND ITS CLOSEST VB (WITH FREQUENCY F3) IS BEING INDICATED BY ITS ASSOCIATED CUES

FIGURE 11 - EXAMPLE OF FFT SPECTRUM AFTER 10S SEGMENTATION OF A SUBTRIAL WHERE THE PARTICIPANT HAD VISUAL FOCUS ON THE VB WITH F2=12,5 Hz. A HIGHER AMPLITUDE PEAK IS FOUND AT THE FUNDAMENTAL FREQUENCY AND A LOWER ONE AT ITS SECOND HARMONIC........................................................................................................................... 47

FIGURE 12 - TEMPLATE WITH THE AVERAGE WAVEFORM OF ONE PARTICIPANT FOR ATTENDED TARGETS............................... 49

FIGURE 13 - CURVES OF THE AVERAGED SEGMENTS OVER 10S (ONE SUBTRIAL) CORRESPONDING TO EACH OF THE FOUR POSSIBLE LOCATIONS (COLOURED CURVES) AND THE TEMPLATE (BLACK CURVE), FOR THE SAME PARTICIPANT AS IN FIGURE 11

FIGURE 14 - SSVEP TASK - AVERAGE ACCURACY (\%) PER CHANNEL AT DIFFERENT SEGMENT LENGTHS - 6 TRIALS (24 CHOICES) PER PARTICIPANT, 19 PARTICIPANTS, IN RED COLOR SCALE. THE ELECTRODE WITH THE HIGHEST ACCURACY IS HIGHLIGHTED (BLUE BORDER) AND THE RESPECTIVE VALUES ARE DISPLAYED INSIDE THEIR ELECTRODES. 55

FIGURE 15- A GRAPHICAL OVERVIEW OF THE RESULTS (USER CHOICE THAT WAS IDENTIFIED BY THE FEATURE DETECTION ALGORITHM) FROM FIVE PARTICIPANTS (FROM LEFT TO RIGHT: P6, P8, P10, P12 AND P14), BASED ON THE OZ CHANNEL, IN COMPARISON TO THE EXPECTED RESULTS (TEMPLATE), USING DIFFERENT SEGMENT LENGTHS (10s, 5s, 2S AND 1S). IN THE PRESENTED TRIAL, THE FREQUENCIES F1 (15Hz), F2 (12,5 Hz), F3 (10,71 Hz) AND F4 (8,33 HZ) WERE INDICATED, IN THIS ORDER, AS CAN ALSO BE OBSERVED IN THE TEMPLATE (FAR LEFT). THE CROSSES IN EACH GRAPH INDICATE THE USER CHOICE THAT WAS IDENTIFIED BY THE FEATURE EXTRACTION ALGORITHM AFTER PROCESSING ONE SEGMENT.

FIGURE 16 - SSVEP TASK: FOOTPRINT OF ACCURACY (\%) PER PARTICIPANT ACROSS ALL ELECTRODES, CONSIDERING ALL 6 TRIALS AND A SEGMENTATION TIME OF 10S. THE HIGHEST ACCURACY CHANNEL(S) ARE HIGHLIGHTED (BLUE) AND THEIR RESPECTIVE VALUES REPORTED IN THE LOWER RIGHT CORNER OF EACH FRAME. UPPER LEFT: AVERAGE ACROSS ALL PARTICIPANTS

FiguRe 17 - SSVEP task: a) Average accuracy (\%) vs. Segmentation time (S) CURVES BaSEd ON OZ CHANNEL ONLY AND ON THE BEST PERFORMING CHANNEL OF EACH PARTICIPANT; B) AND C) CURVES OF EACH PARTICIPANT FOR BOTH AVERAGE CURVES PRESENTED IN A).

FIGURE 18- P300 TASK: AVERAGE ACCURACY (\%) PER CHANNEL AT DIFFERENT SEGMENTATION LENGTHS - 6 TRIALS (24 CHOICES) PER PARTICIPANT, 19 PARTICIPANTS. THE ELECTRODE WITH THE HIGHEST ACCURACY IS HIGHLIGHTED (BLUE BORDER) AND THE AVERAGE ACCURACY VALUES ARE DISPLAYED IN EACH ELECTRODE 61

FIGURE 19 - P300 TASK: FOOTPRINT OF ACCURACY (\%) PER PARTICIPANT, CONSIDERING ALL 6 TRIALS AND SEGMENTATION TIME OF 10S. THE HIGHEST ACCURACY CHANNEL(S) ARE HIGHLIGHTED (BLUE) AND THEIR RESPECTIVE VALUES REPORTED ON THE LOWER RIGHT CORNER OF EACH FRAME. UPPER LEFT: ALL PARTICIPANTS AVERAGE

Figure 20- P300 task: Accuracy (\%) vs. Segmentation time (s) CURVES BASEd on Po8 CHANNEl ONLY AND ON the BEST PERFORMING CHANNEL OF EACH PARTICIPANT, FOR BOTH FEATURE EXTRACTION ALGORITHMS "PEAK TO PEAK" AND "TEMPLATE MATCH"; 
FIGURE 21 - P300 TASK: ACCURACY (\%) ACHIEVED BY EACH PARTICIPANT BASED ON THEIR BEST PERFORMING CHANNEL AND 10S SEGMENTATION, FOR BOTH FEATURE EXTRACTION ALGORITHMS. 65

FiguRE 22 - HYBRID TASK: AVERAGE ACCURACY (\%) PER CHANNEL AT 10S SEGMENTATION - 6 TRIALS (24 CHOICES) PER PARTICIPANT, 19 PARTICIPANTS. THE ELECTRODES WITH THE HIGHEST ACCURACY ARE HIGHLIGHTED (BLUE BORDER) AND THE AVERAGE ACCURACY VALUES ARE DISPLAYED IN EACH ELECTRODE. 66

FigURE 23 - ALL TASKS: AVERAGE ACCURACY (\%) VS. SEGMENTATION TIME (S) CURVES BASED ON THE BEST PERFORMING CHANNEL OF EACH PARTICIPANT.

FIGURE 24 - ALL TASKS: ACCURACY (\%) ACHIEVED BY EACH PARTICIPANT BASED ON THEIR BEST PERFORMING CHANNEL AND 10S SEGMENTATION. 68

FIGURE 25 - ITR** VS. SEGMENTATION TIME CURVES FROM SSVEP AND P300 TASKS, BASED ON THE AVERAGE ACCURACIES AND ON THE MAXIMUM ACCURACIES ACHIEVED BY ONE (OR MORE) PARTICIPANT(S), FOR DIFFERENT SEGMENTATION LENGTHS 71

FiguRE 26 - VISUAL REPRESENTATION OF BEST PERFORMING SETS OF 8, 16 AND 32 CHANNELS PER TASK, ACCORDING TO THEIR AVERAGE ACCURACIES. THE RANKING POSITIONS ARE DISPLAYED INSIDE THEIR RESPECTIVE ELECTRODES...... 76 FIGURE 27 - LAYERS OF NON-NEURAL TISSUES BETWEEN ACTIVE SYNAPSES AND THE EEG ELECTRODE (BEAR, CONNORS AND PARADISO 2016)

FIGURE 28 - ELECTRODES POSITIONING OVER THE SCALP BASED ON THE INTERNATIONAL 10-20 SYSTEM (SANEI AND CHAMBers 2007) 


\section{LIST OF TABLES}

TABLE 1 - ITR RESULTS FROM ALL TASKS, BASED ON THE HIGHEST ACCURACIES ACHIEVED (10S SEGMENTATION) 69

TABLE 2 - ITR** RESULTS FROM SSVEP AND P300 TASKS, BASED ON THE AVERAGE ACCURACIES AND ON THE MAXIMUM ACCURACIES ACHIEVED BY ONE (OR MORE) PARTICIPANT(S), FOR DIFFERENT SEGMENTATION LENGTHS. 70

TABLE 3 - NUMBER AND PERCENTAGE OF ILLITERATES PER TASK FROM A TOTAL OF 19 PARTICIPANTS. 72

TABLE 4 - RESULTS OF THE INDIVIDUAL PREFERENCE ASSESSMENT BASED ON QUESTIONNAIRES COMPLETED AFTER THE EXPERIMENT. AFTER THE EXPERIMENT, THE PARTICIPANTS WERE ASKED HOW THEY AGREED WITH THE STATEMENT "I LIKED TO PERFORM THE TASK X", FOR EACH TASK, ACCORDING TO THE LIKERT SCALE: -2: STRONGLY DISAGREE, -1: SLIGHTLY DISAGREE, 0: NEUTRAL AGREEMENT, 1: SLIGHTLY AGREE AND 2: STRONGLY AGREE. 73

TABLE 5 - FULL RANKING OF ALL 62 CHANNELS PER TASK, ACCORDING TO THEIR RESPECTIVE AVERAGE ACCURACIES. ......... 73 TABLE 6 - AVERAGE ACCURACY CONSIDERING THE BEST PERFORMING ELECTRODE PER PARTICIPANT FROM THE FULL SET OF 62 ELECTRODES AND REDUCED SETS OF THE BEST PERFORMING 32, 16 AND 8 ELECTRODES, ACCORDING TO THE RANKING PRESENTED IN TABLE 5. 


\section{LIST OF ABBREVIATIONS}

$\mathrm{BCl} \quad$ Brain Computer Interface

BMI Brain Machine Interface

CNS Central Nervous System

EEG Electroencephalography

ERD Event Related Desynchronization

ERS Event Related Synchronization

fNIRS functional Near Infrared Spectroscopy

ISI Inter Stimulus Interval

ITR Information Transfer Rate

SSVEP Steady State Visual Evoked Potential 


\section{SUMMARY}

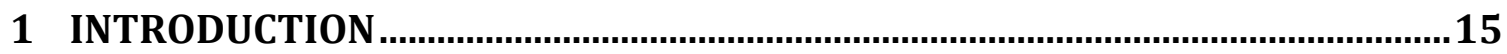

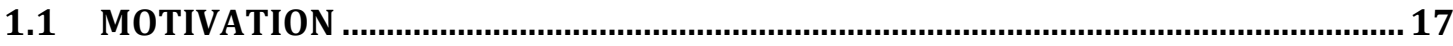

2 STATE OF THE ART

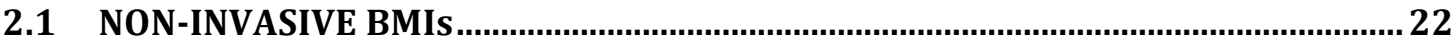

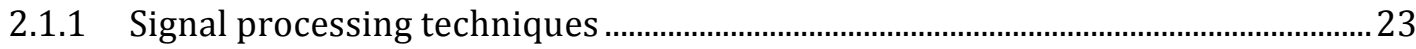

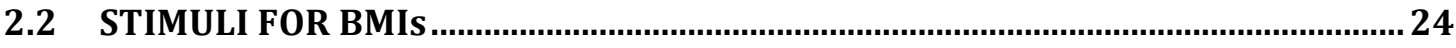

2.2.1 Steady State Visual Evoked Potential (SSVEP) ……...................................................26

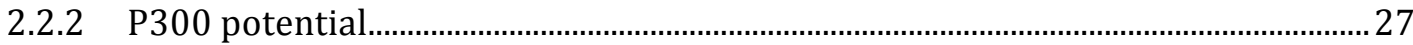

2.2.3 Sensorimotor cortex activity and ERD/ERS ………...................................................2

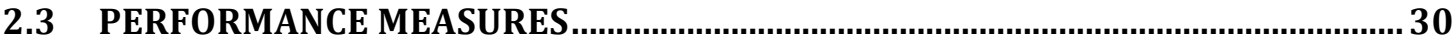

2.4 REAL-LIFE APPLICATIONS VERSUS STUDIES LIMITATIONS ..................................31

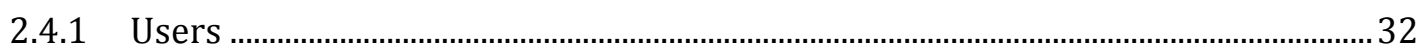

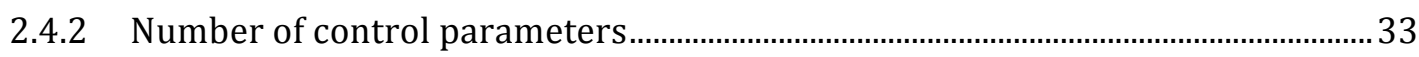

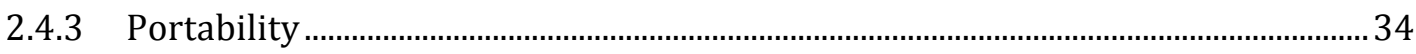

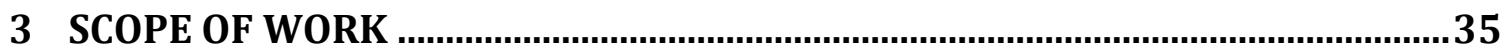

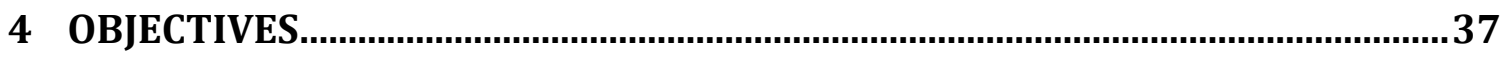

5 METHODS

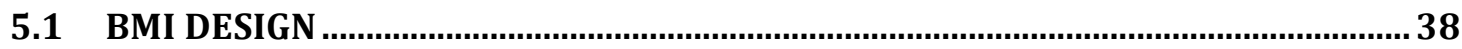

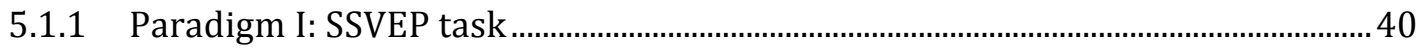

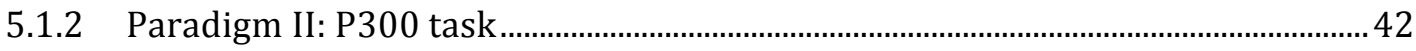

5.1.3 Paradigm III: Hybrid task

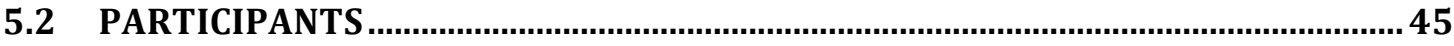

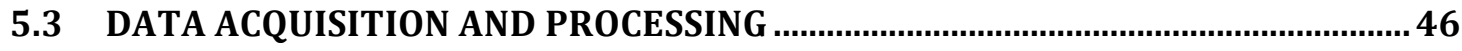

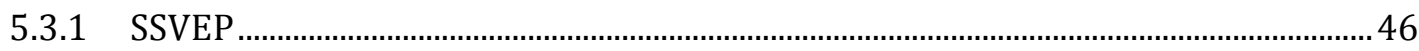

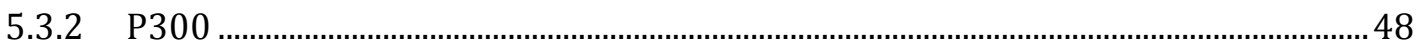

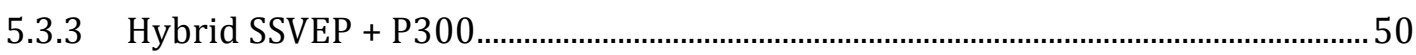

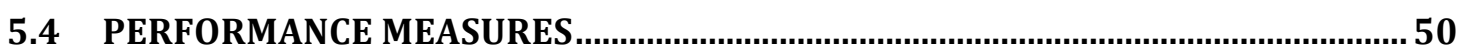

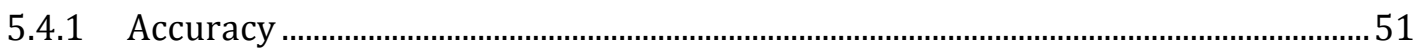

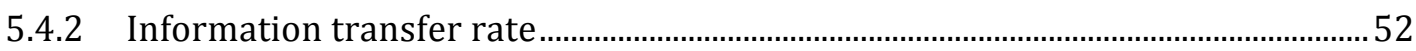

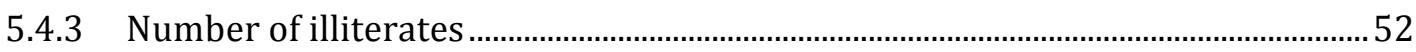

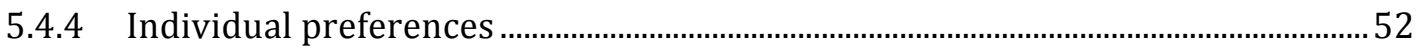

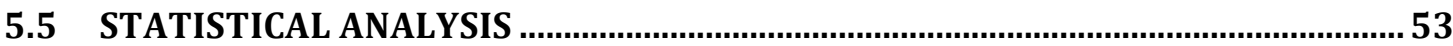

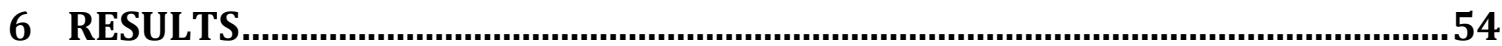


6.1 ACCURACY

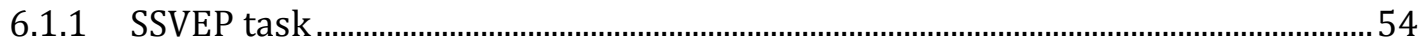

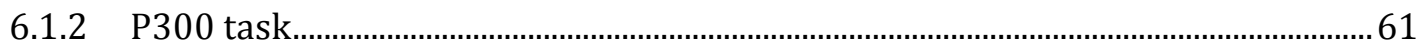

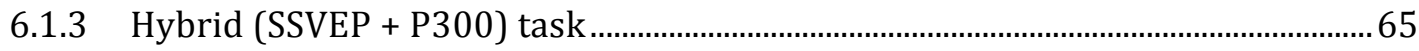

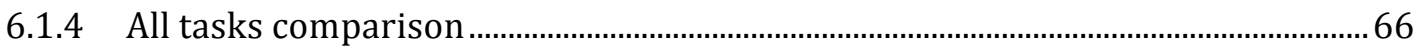

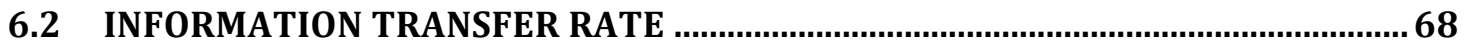

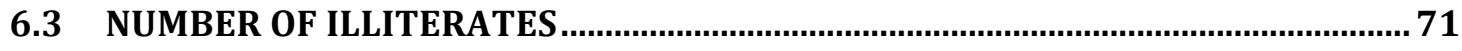

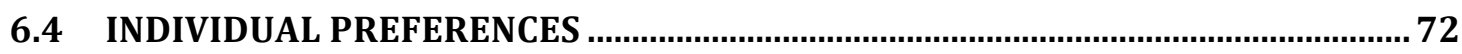

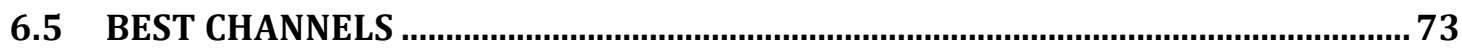

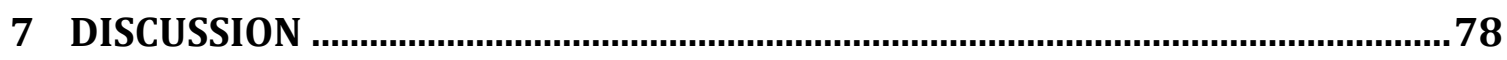

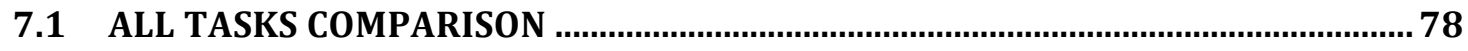

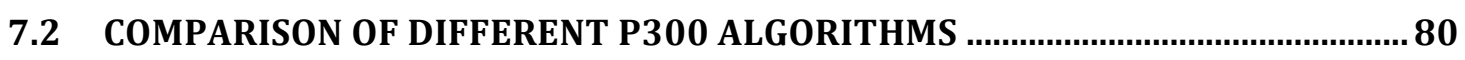

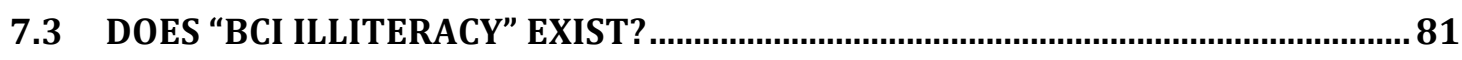

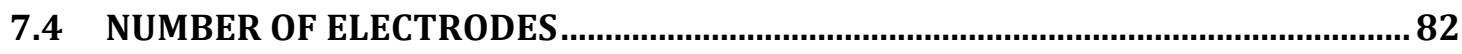

7.5 FINAL CONSIDERATIONS AND POSSIBLE FUTURE DEVELOPMENTS .................. 82

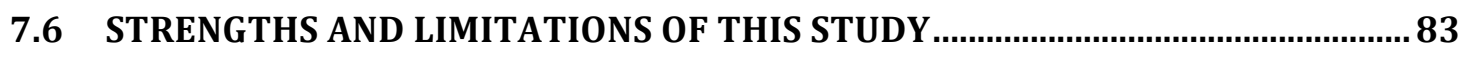

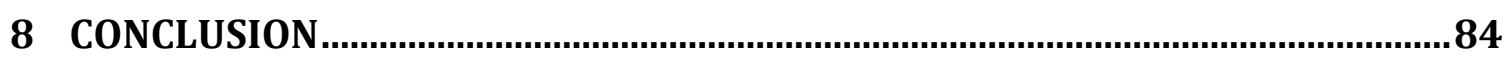

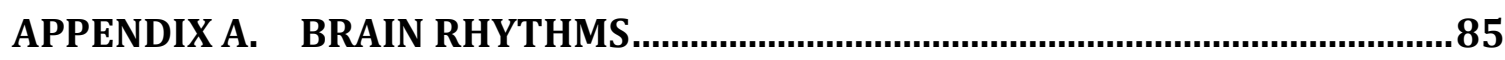

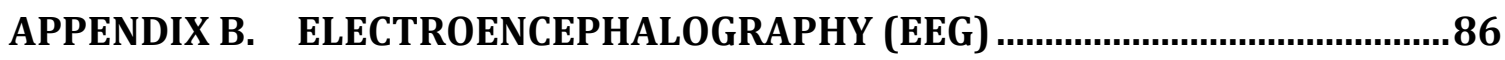

APPENDIX C. NEAR INFRARED SPECTROSCOPY (NIRS) ...................................... 89

ANNEX A MATLAB SCRIPT: SSVEP TASK ........................................................... 91 


\section{INTRODUCTION}

A brain-machine interface (BMI), also known as brain-computer interface $(\mathrm{BCl})$, can be generically defined as a communication interface system between the central nervous system (CNS) and an external device. As a potential assistive technology to paralyzed or 'locked-in' individuals suffering from motor control disorders, a BMI could be designed to translate the brain activity into control commands for an external device as a computer cursor control, an external actuator or a prosthetic arm, to name a few (Wolpaw, Birbaumer, McFarland, Pfurtscheller and Vaughan 2002). However, possible applications are not restricted to severely disabled users and, more recently, (Wolpaw and Wolpaw 2012) defined a BCl as follows: "a system that measures CNS activity and converts into artificial output that replaces, restores, enhances, supplements or improves natural CNS output and thereby changes the ongoing interactions between the CNS and its external or internal environment" (see Figure 1).

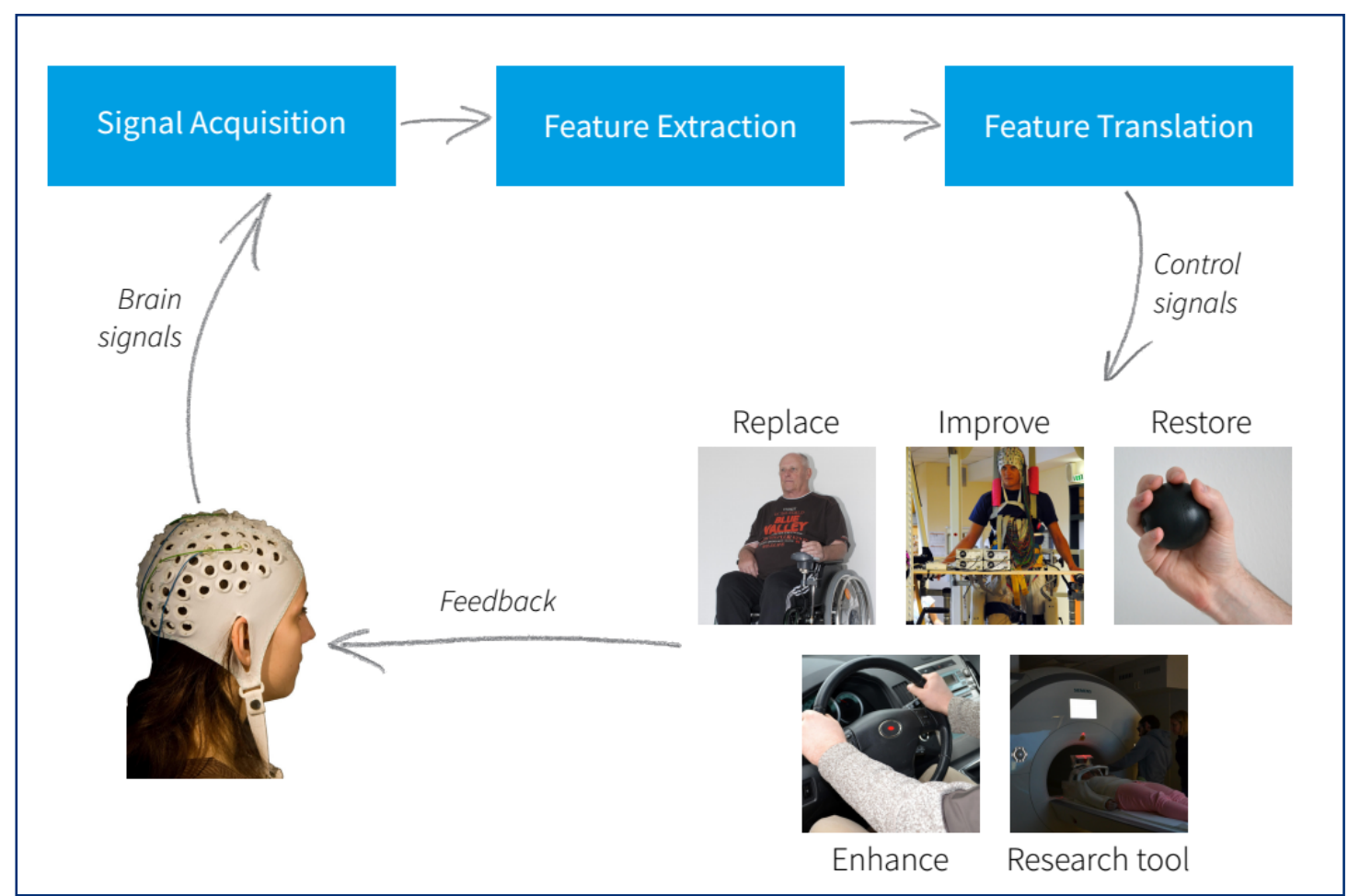

Figure 1 - Principle of a brain-computer interface including possible application scenarios ((Brunner, Birbaumer, Blankertz, Guger, Kübler, Mattia, Millán, Miralles, Nijholt, Opisso, Ramsey, Salomon and Müller-Putz 2015), adapted from (Wolpaw and Wolpaw 2012)). 
Currently, $\mathrm{BCl}$ and $\mathrm{BMI}$ terms are synonymous terms (Wolpaw and Wolpaw 2012) and the choice between them is mostly a matter of personal preference. In this study, the term BMI will be used, unless we are referring to another study where the author's choice was to use the term BCl.

The following stages (or a subset of them) are typically involved (Rao 2013) in a generic BMl:

a) Brain stimulation: the brain activity is externally or spontaneously stimulated;

b) Brain recording: signals from the brain are recorded using either invasive or non-invasive recording techniques;

c) Signal processing: raw signals are preprocessed (e.g. by band pass filtering) and techniques for artifact reduction and feature extraction are used;

d) Pattern recognition and machine learning: a control signal based on patterns is generated in the input, typically using machine-learning techniques;

e) Sensory feedback: the control signal from the BMI causes a change in the environment (e.g. movement of a prosthetic arm). Sensors, cameras and displays can be used and integrated to provide visual, auditory or tactile feedback to the user.

The BMIs can be classified according to several characteristics (Nicolas-Alonso and Gomez-Gil 2012; Wolpaw et al. 2000):

a) Brain signal recording technique: (i) invasive or (ii) non-invasive. Invasive techniques are based on microelectrodes that are implanted in the brain or placed on the surface of the brain and may allow recordings from individual neurons. Non-invasive techniques do not require surgical procedures and record electrical activity of large populations of neurons (using electrodes placed on the scalp), changes in blood oxygenation level that result from neural activity or magnetic field fluctuations caused by electrical activity from the brain;

b) Brain activity measured: (i) electrophysiological or (ii) hemodynamic. BMls based on electrophysiological activity detect changes in the electrical potentials of neurons or populations of neurons. BMls based on hemodynamic activity detect changes in the hemodynamic response, a process in which the blood releases glucose to active neurons at a greater rate than in the area of inactive neurons; 
c) Brain stimulation method: (i) exogenous or (ii) endogenous (Nicolas-Alonso and Gomez-Gil 2012). Exogenous BMls use the brain activity elicited by an external stimulus as (e.g. steady state visually evoked potential (SSVEP)) and may not require extensive training but do require some structured environment and permanent attention to the external stimuli. On the other hand, endogenous systems are based on the self-regulation of brain rhythms and potentials (e.g. slow cortical potentials and sensorimotor rhythms) learned through extensive neurofeedback training (see APPENDIX A. BRAIN RHYTHMS);

d) Input data processing modality: (i) synchronous (cue-paced) or (ii) asynchronous (self-paced). Synchronous BMls analyse brain signals during predefined time windows while asynchronous BMls analyse brain signals continuously;

e) Translation algorithm: (i) discrete or (ii) continuous. The output of the BMI translation algorithm may be discrete (e.g. letters from the alphabet) or continuous (e.g. cursor movement).

\subsection{MOTIVATION}

There are limitations to be overcome before a successful implementation of a BMI for the control of an external device, such as an exoskeleton or a prosthetic device is achieved in a real-life environment. In general, the challenge consists of reaching a compromise between the pros and cons that are intrinsic to characteristics such as invasiveness level, health risk, signal-to-noise ratio, acquisition area, user controllability of the area, rate of information transfer, cognitive workload, stability, portability and cost.

Invasive modalities as electrocorticography $(\mathrm{ECoG})$ or intracortical neuron recording allow direct measurement of the electrical activity through implanted electrodes on the surface or inside the cortex, offering the best temporal and spatial resolution, simultaneously. Although progress on implant technologies to improve biocompatibility have been made, those techniques still entail high health risks such as infection or rejection and the number of reliably functional electrodes drops 
substantially and continuously after a few weeks or months post-implantation. In the long term, keeping the communication between the electrodes and the external hardware and, intrinsically, a high performance, is a real challenge (Nicolas-Alonso and Gomez-Gil 2012; Schwartz et al. 2006).

Non-invasive modalities can be adapted to be safely used outside the experimental environment but, as the brain activity measurement is indirect, the desired information is attenuated and distorted through the tissues, becoming less differentiable from noise and other artifacts (Tong et al. 2011). As a result, time and spatial resolution are worsened. Electroencephalography (EEG) is the most widespread non-invasive modality for brain activity recording for BMls (see APPENDIX B. ELECTROENCEPHALOGRAPHY (EEG)). Electrodes are placed on the scalp to measure the electrical signals that are generated from depolarization of neurons in the brain and cross the superficial layers of the head, including scalp and skull. The resulting signals are weak and highly affected by background noise.

Functional near infrared spectroscopy (fNIRS) is another non-invasive modality for brain activity measurement that has been increasingly used in BMI studies (Cutini and Brigadoi 2014; Matthews et al. 2008; Scholkmann et al. 2014; Sitaram et al. 2009). FNIRS is able to capture the hemodynamic changes via the coupling of infrared light emission and detection (see APPENDIX C. NEAR INFRARED SPECTROSCOPY (NIRS)). Since the cortical region of the brain contains relevant information about motor cortex activity, strategies that involve its stimulation are commonly used for the control of fNIRS-based BMls (Aqil et al. 2012; Matthews, Pearlmutter, Ward, Soraghan and Markham 2008). The hemodynamic response has an intrinsic onset delay of at least 1-2 $\mathrm{s}$ with respect to its associated electrical response from neural activity (Coyle et al. 2007; Fazli et al. 2012; Strait and Scheutz 2014b). However, fNIRS has been reported to achieve better overall discrimination between two workload levels in comparison to EEG, at certain stimuli presentation conditions (Putze et al. 2014). Additionally, the hemodynamic activity information is complementary to the electrical activity information provided by EEG. The idea of using fNIRS for an optical BMI system has been first introduced by (Coyle et al. 2004) and then followed by other groups (Abdelnour and Huppert 2009; Bauernfeind et al. 2008; Coyle, Ward and Markham 2007; Luu and Chau 2009; Sitaram et al. 2007). Later, a hybrid BMI concept combining at least one direct brain signal to additional inputs (such as fNIRS to function as a brain switch to control a hand 
orthosis) was conceptualized and proposed by (Pfurtscheller et al. 2010), being further studied by other groups (Blokland et al. 2014). A hybrid BMI approach based on simultaneous measurement of EEG and fNIRS for Sensory Motor Rhythm (SMR)based BMI paradigm was proposed by (Fazli, Mehnert, Steinbrink, Curio, Villringer, Müller and Blankertz 2012).

While the simultaneous use of fNIRS and EEG to achieve higher classification rates for certain paradigms have been demonstrated, it remains to-date unclear how much this multimodal approach can improve a BMI performance.

Classification accuracy above $70 \%$ is often considered in the literature as the minimum performance requirement for a BMI for communication establishment and user controllability (Pfurtscheller, Allison, Brunner, Bauernfeind, Solis-Escalante, Scherer, Zander, Mueller-Putz, Neuper and Birbaumer 2010; Power et al. 2012). A standard method for BMl performance evaluation incorporating both speed and accuracy in one single value that can be defined as the information transfer rate, or bit rate (in bits/trial or bits/min) was proposed by (Wolpaw, Birbaumer, Heetderks, McFarland, Peckham, Schalk, Donchin, Quatrano, Robinson and Vaughan 2000; Wolpaw, Birbaumer, McFarland, Pfurtscheller and Vaughan 2002). Based on previous work on Information Theory (Pierce 1980), the bit rate B (in bits/trial) can be computed as:

$$
B=\log _{2} N+P \log _{2} P+(1-P) \log _{2}\left[\frac{(1-P)}{N-1}\right]
$$

where $\mathrm{N}$ is the number of possible choices and $\mathrm{P}$ is the correct choice probability. The bit rate in bits/min can be computed by dividing $B$ by the trial duration in minutes. (Wolpaw, Birbaumer, Heetderks, McFarland, Peckham, Schalk, Donchin, Quatrano, Robinson and Vaughan 2000; Wolpaw, Birbaumer, McFarland, Pfurtscheller and Vaughan 2002) estimated that the maximum information transfer rate achieved by a BMI was around $25 \mathrm{bits} / \mathrm{min}$. Although the transfer rate depends on the complexity level of the desired task, it is estimated that a very complex task such as brain-tospeaking requires a transfer rate as high as $2400 \mathrm{bits} / \mathrm{min}$ (Tehovnik and Chen 2015) and, as it has been suggested in a recent review (Tehovnik et al. 2013), BMI transfer rates have not consistently improved over the last decade.

Moreover, other factors beyond the information transfer rate must be considered in order to objectively compare the different systems. Advantages and disadvantages of different signal acquisition and feature extraction methods are still not clear and 
require more studies. Different equipment, setups, sessions, system architectures, environments, brain stimuli, signal processing techniques, users' abilities or physical characteristics, among other factors, may affect the performance results. Also, some users are currently classified as BMI illiterates, as they are not able to control a BMI after several training sessions.

In spite of several attempts and initiatives from BMI groups and members over the last two decades to provide standardization guidelines, the cross comparison between studies and different BMls remains a challenge (Thompson, Quitadamo, Mainardi, Laghari, Gao, Kindermans, Simeral, Fazel-Rezai, Matteucci, Falk, Bianchi, Chestek and Huggins 2014).

Therefore, this study intends to overcome part of the challenges related to the acquisition and characterization of brain signals for BMI applications, contributing to the technological advancement of this research and, indirectly, for the greater welfare and greater active participation of disabled people in society. 


\section{STATE OF THE ART}

Firstly, this study was limited to healthy participants and to non-invasive technologies that do not entail health risks to the users. Then, an extensive bibliographic review (Villalpando, M. B.; Fukasawa, A.; Forner-Cordero, A., "Noninvasive Brain-machine interfaces: a review from a system perspective", submitted) has been performed to map the state-of-the-art of non-invasive BMIs based on fNIRS and/or EEG and their respective aspects affecting system performance.

A summary table was prepared to organize the experimental paradigm, brain activation area, signal acquisition equipment, electrodes/optodes placement setup and signal processing techniques used in each article in a comprehensive way. The comparison of the performance across the studies was not possible due to the lack of standardization and homogeneity both in the measurements and in the experimental setups.

Based on this review, possible solutions that have been or could be used to enhance the performance of BMIs, within the scope of non-invasive modalities, are:

a) Implementation of a hybrid system obtaining signals from the CNS, but of different natures (such as a combination of hemodynamic activity using fNIRS and electrical activity using EEG);

b) Implementation of a hybrid system obtaining signals from the CNS, but based on different brain stimuli (such as a combination of P300 and SSVEP and/or ERD/ERS);

c) Inclusion of physiological signals not directly derived from brain activity (such as eye-trackers, heart or respiration rate monitors) in the system, for user's monitoring, filtering and/or artifacts removal;

d) Model improvements based either on (i) the actual brain dynamics or (ii) brain response functions, for output information prediction and/or processing optimization.

Further, BMls are control systems intended to be used in real-life applications. Therefore, the future BMls should be more integrated to the environment and autonomous, reducing the control burden on the human side. Thus, there are several aspects that affect the BMI performance, either directly or indirectly, and the ITR is not sufficient to objectively compare the different systems. 
The following subsections provide an overview of the state-of-the art on noninvasive BMIs, the main aspects elements involved in the design of a BMI system, the performance measures currently in use and the challenges for real-life applications development.

\subsection{NON-INVASIVE BMIS}

As mentioned in section 1.1, EEG is the most widespread non-invasive modality for BMIs brain activity recording and fNIRS is another modality that is increasingly being used. It is possible to build a multimodal BMI, also known as hybrid BMI, combining inputs from EEG, NIRS, electrophysiological sensors (i.e. EMG, ECG) or external devices (i.e. eye tracking system), but at least one input must be obtained from brain signals (Pfurtscheller, Allison, Brunner, Bauernfeind, Solis-Escalante, Scherer, Zander, Mueller-Putz, Neuper and Birbaumer 2010).

In case of fNIRS-based BMls, it must be considered that the hemodynamic response is delayed by $1-3 \mathrm{~s}$ to the neuronal event and its peak is reached after $5-8 \mathrm{~s}$ (Naseer and Hong 2013; Tomita et al. 2014). Moreover, there is a hemodynamic delay between $\mathrm{HbO}$ and $\mathrm{HbR}$ (Boden et al. 2007) and, therefore, depending on which data is being acquired or compared, a lag up to 2 seconds must the taken into account. This intrinsically slow characteristic from the hemodynamic response limits the interest in single fNIRS signal measurement for BMI applications (Power et al. 2010; Strait and Scheutz 2014a; Tomita, Vialatte, Dreyfus, Mitsukura, Bakardjian and Cichocki 2014) and also raises the concern on the benefit of its employment on hybrid EEG-fNIRS based BMIs, in spite of the demonstration of its positive results on classification accuracies over different paradigms. Furthermore, the lag between activities of different natures and sources (electrical and hemodynamic, for example) results in delays for the BMI system (Takeuchi et al. 2009; Tong and Frederick 2010; Tong et al. 2012).

However, the purpose of a multimodal approach is not necessarily to improve accuracy by increasing the information rate and types. Multimodal information can also be used to: a) detect resting (break) periods when it is not desired that the BMI operates, b) to anticipate upcoming activity, c) to reduce the BMI illiterates number 
(Blokland, Spyrou, Thijssen, Eijsvogels, Colier, Floor-Westerdijk, Vlek, Bruhn and Farquhar 2014). The strategy to process the inputs in a hybrid BMI can be either simultaneous or sequential (e.g. as a brain switch) (Pfurtscheller, Allison, Brunner, Bauernfeind, Solis-Escalante, Scherer, Zander, Mueller-Putz, Neuper and Birbaumer 2010).

From the anatomic perspective, the International 10-20 system is the standard for electrodes placement in EEG and is also a reference that is commonly followed for optodes placement in fNIRS. However, in spite of the efforts from some groups (Funane et al. 2012; Nakai et al. 2008; Okamoto et al. 2004; Selb et al. 2014; Stuban et al. 2012; Tian and Liu 2014), an equivalent probe placement system has not been developed for fNIRS and cross-comparison of the studies becomes very difficult.

\subsubsection{Signal processing techniques}

In the BMI context, the signal processing should enable the interface system to gather useful information from the recorded signals to operate the interface that controls the machine.

Pre-processing is required, since the recorded raw data is usually not ready to be processed. In both EEG and fNIRS acquisition cases, the acquired data is composed not only of brain activity related to the task of interest, but also of another signals such as blood circulation in the scalp, motion artifacts and others. Data down sampling, band pass filtering techniques are commonly used in both EEG and fNIRS pre-processing step.

There are several methods of signal processing that can be applied, either based on time domain analysis or frequency domain analysis. Fast Fourier Transform, Wavelet Analysis, Spatial Filtering Techniques, Common Average Referencing, Rereferencing, among several other possibilities that can be also combined together have been used in different BMI studies. Within the scope of the frequency domain analysis, the Fourier Transform is a mathematical tool that decomposes the signal into a sum of sines and cosines that represent different frequencies. However, the Fourier Transform presents a major drawback in the trade-off between frequency and time resolution, as the duration of the recording limits the frequency resolution: long 
time recordings may improve the frequency resolution, but limit the time resolution. In other words, using the Fourier Transform it may be possible to find which frequencies compose the signal, but it may not be possible to know when they occurred.

For online implementation using fNIRS, Bayesian filters (e.g. Kalman filter) have been gaining popularity and present promising results (Abdelnour and Huppert 2009; Gagnon et al. 2011). Importantly, different basic assumptions should be made if Gaussian random field theory is used for signal analysis due to the different properties of hemodynamic and electrical activities (Koh et al. 2007; Machado et al. 2011; Ye et al. 2009).

Besides, machine learning techniques have been successfully applied to reduce the effects of the different sources of variability in the measured signals, thereby increasing the BMI robustness and reducing the required training (Abibullaev and An 2012; Müller et al. 2008).

Thus, by discussing the processing techniques mentioned above, it can be observed that there is no processing technique that fits all the problems. The correct choice on depends on the expected effects on the cortical activity according to the brain stimuli used and the specificities of each modality.

\subsection{STIMULI FOR BMIs}

A broad variety of experimental paradigms can be found in the literature, to elicit cortical activity:

a) Visual tasks involve the presentation of visual stimuli such as reversing checkerboards on a screen (Chen et al. 2014; Sun et al. 2014) and are associated with visual cortex activation, known as visual evoked potential (VEP);

b) Auditory tasks consist in the presentation of auditory stimuli such as tones at specific frequencies, intensities and durations (Chen et al. 2015) and are associated with auditory cortex activation, known as auditory evoked potential (AEP);

c) Motor tasks comprise either imagined or real movements and motor imagery can achieve similar, though weaker, cortical activation patterns if compared to the actual movements (Blokland, Spyrou, Thijssen, Eijsvogels, Colier, Floor-Westerdijk, Vlek, Bruhn and Farquhar 2014; Yin et al. 2015); 
d) Cognitive tasks can be, for example, mental arithmetic, reading or verbal fluency tasks (VFT) (Kameyama et al. 2004; Uehara et al. 2007). They are associated with pre-frontal cortex activation and the activation level may vary according to the complexity level (Abibullaev and An 2012; Tachibana et al. 2011);

e) Emotionally charged task typically consist of the presentation of images that induce an emotional impact on the user, being the International Affective Picture the most endorsed tool for exploring the emotional processing and appraisal of emotional cues. According to recent studies, the emotional response has been associated with pre frontal cortex activation (Strait and Scheutz 2014b) and lateralization (Balconi et al. 2015). However, there is no agreement about a normative activation pattern or about the emotional model based on valence and arousal.

The successful control of a BMI depends both on the modulation of the activity of some specific cortex area(s) of the user (see Figure 2) and on the BMI system acquisition and processing of the brain signals that convey this information, detecting them and translating them into commands to activate an external device.

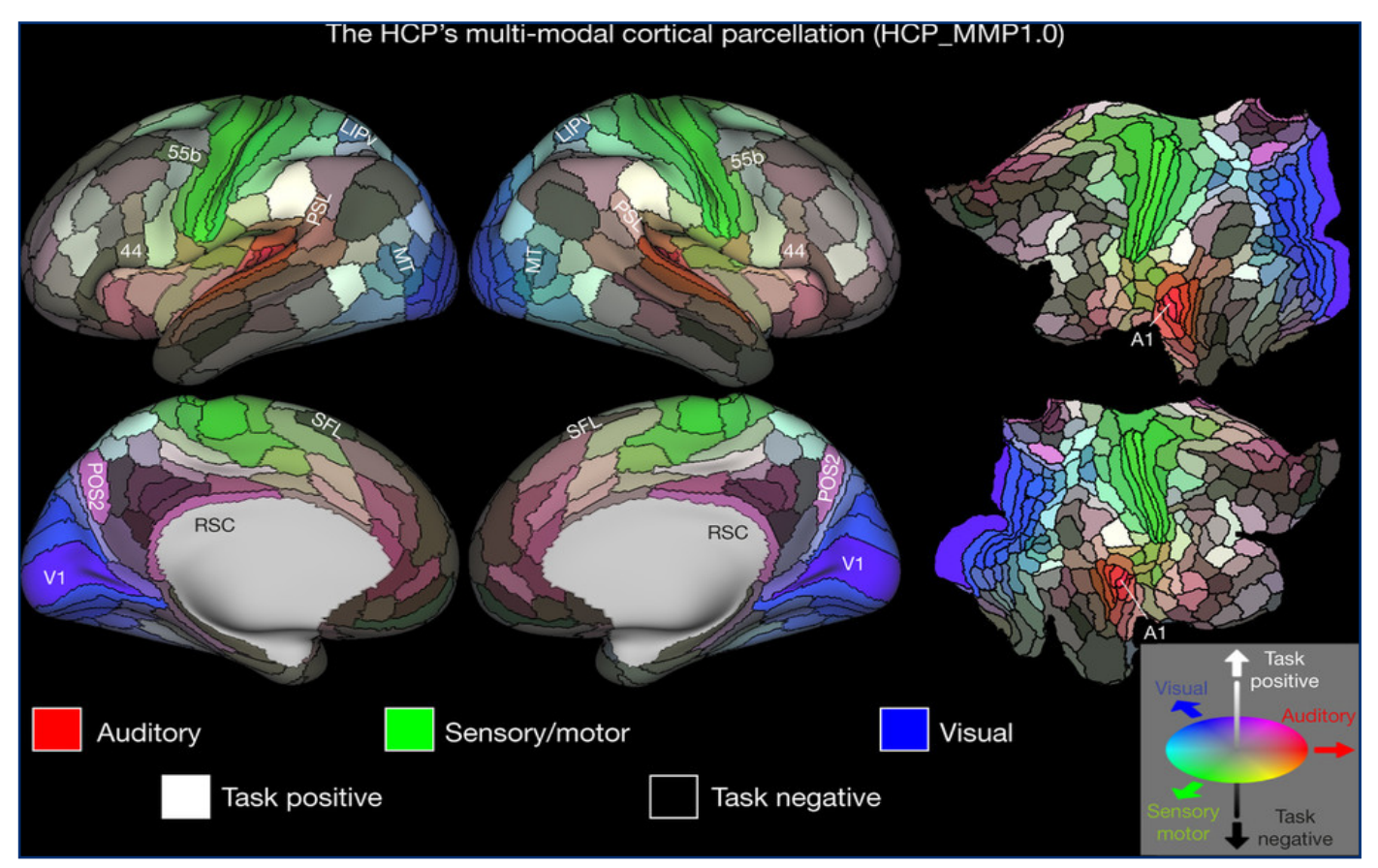

Figure 2 - The HCP's multi-modal parcellation, version 1.0 (HCP_MMP1.0): The 180 areas delineated and identified in both left and right hemispheres are displayed on inflated and flattened cortical surfaces. Black outlines indicate areal borders. Colours indicate the extent to which the areas are associated in the resting state with auditory (red), somatosensory (green), visual (blue), task positive (towards white), or task negative (towards black) groups of areas. The legend on the bottom right illustrates the 3D colour space used in the figure. Data at http://balsa.wustl.edu/WN56. (Glasser et al. 2016) 
Moreover, it should be considered that long and exhausting trials can lead to physical and mental fatigue of the user and loss of attention (Tai and Chau 2009). Thus, it is desirable that the task can be easily learned and executed, avoiding both strenuous training sessions and user tiredness while operating the system. In other words, the task selection involves a compromise between brain activation intensity and continuous operation time of the system.

Therefore, the proper selection of a task or of an external stimulus that can elicit a detectable cortical response is imperative to obtain a BMI. This section provides an overview of some brain stimuli and how they have been used for BMls, as a foundation to the methods used in the proposed study.

\subsubsection{Steady State Visual Evoked Potential (SSVEP)}

The SSVEP is the response elicited in populations of neurons in the visual cortex when the user is fixating on a visual stimulus (e.g., a checkerboard pattern) flickering at a particular frequency. The associated brain signal exhibits peaks in the power spectrum at the stimulus frequency and its harmonics (see Figure 3). SSVEP occurs naturally with stimulation but, with biofeedback training, users can also learn to wilfully control their SSVEP amplitude (Middendorf et al. 2000). The communication between the user and the computer is binary (the response is present or absent), being appropriate to describe this $\mathrm{BMI}$ as a discrete controller. If different frequencies are associated with different choices, an SSVEP-based BMI can decode the user's choice by detecting where the peaks are. A typical task consists of selecting a flickering icon on a computer screen simply by looking at it. Several icons flickering at different frequency rates can be presented at the same time (Middendorf, McMillan, Calhoun and Jones 2000).

Although SSVEP responses can be induced by frequencies ranging from 1 to $100 \mathrm{~Hz}$, several studies have concluded that the most effective frequencies for BMI applications belong to the low-frequencies range from 6 to $18 \mathrm{~Hz}$ (Gao et al. 2003; Kuś et al. 2013; Volosyak 2011). 


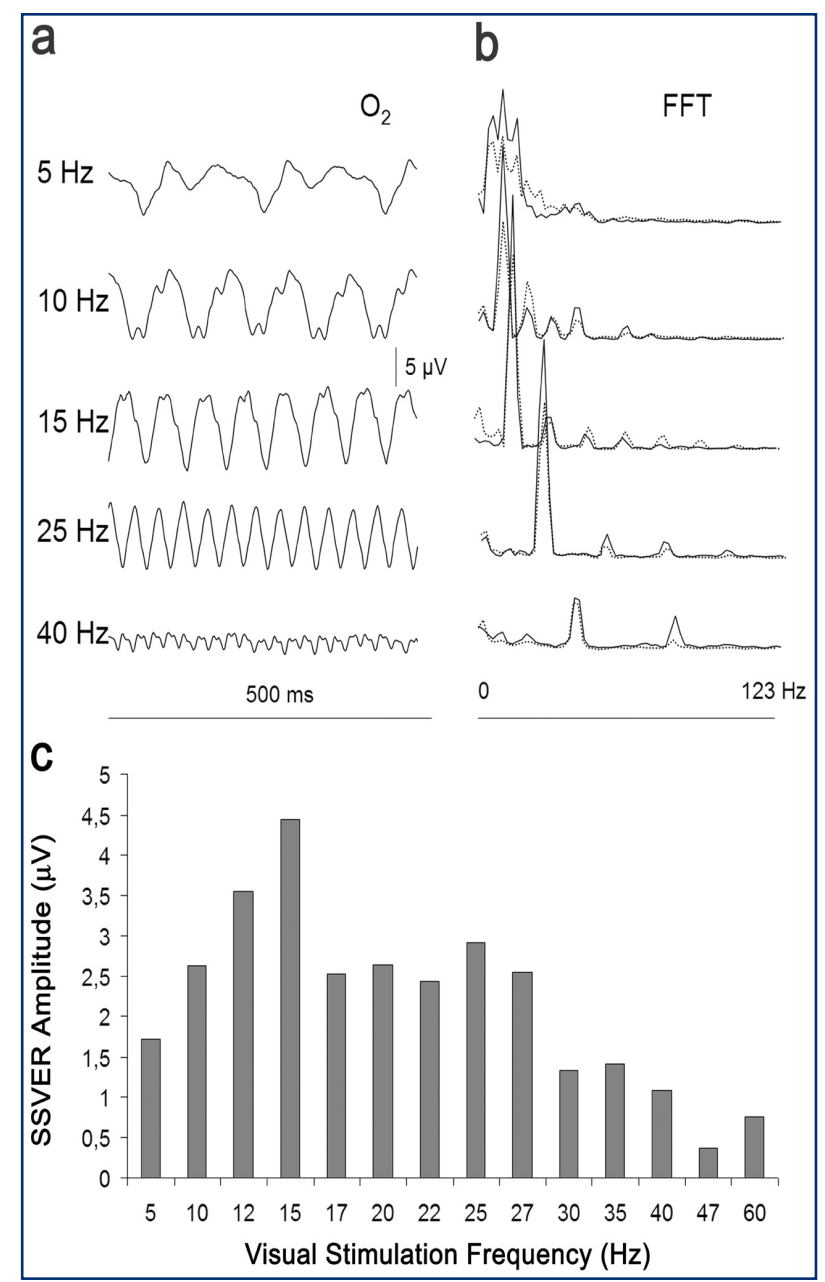

Figure 3 - Effect of visual stimulation at different frequencies on the amplitude of the SSVER. A) SSVER recordings of one representative subject at the right occipital lead (O2). B) FFT of this individual's SSVERs and grand average of 16 normal subjects (dotted line). C) Average of the mean values of the amplitude of the FFT fundamental frequency of the SSVER recorded at the three occipital leads $(\mathrm{Oz}$, center; O1, left; O2, right) at the different stimulation frequencies. The amplitude of the occipital SSVER, expressed in microvolts, reached a maximum at $\sim 15 \mathrm{~Hz}$ and then fell with a plateau up to $27 \mathrm{~Hz}$, declining at higher frequencies (Pastor et al. 2003).

\subsubsection{P300 potential}

The P300 is an example of an ERP that is evoked by the occurrence of a rare (less probable) stimulus and appears as a positive deflection in the EEG signal with a latency of approximately $300 \mathrm{~ms}$. It is most frequently elicited within the framework of what has come to be called the "oddball paradigm" where the user is presented a sequence of events that can be classified into two categories and events in one of 
the two categories are rarely presented. Events in the rare, or less probable, category will elicit an ERP (see Figure 4) which is generally observed most strongly over the parietal lobe, although some components also originate in the temporal and frontal lobes (Donchin et al. 2000; Rao 2013).

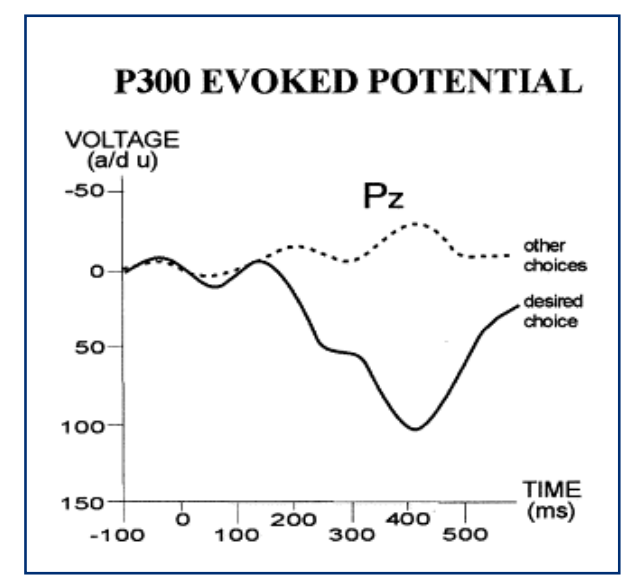

Figure 4 - $\mathrm{P} 300 \mathrm{BCl}$. A matrix of possible choices is presented on a screen and scalp EEG is recorded over the centroparietal area while these choices flash in succession. Only the choice desired by the user evokes a large P300 potential (i.e. a positive potential about $300 \mathrm{~ms}$ after the flash) (adapted from (Donchin, Spencer and Wijesinghe 2000; Wolpaw, Birbaumer, McFarland, Pfurtscheller and Vaughan 2002)).

The original P300-based BCl that was described by (Farwell and Donchin 1988), which is also called the "P300 Speller", presented a $6 \times 6$ matrix of characters representing letters and commands to a user. The rows and the columns were intensified (or highlighted) in a random sequence in such a manner that all 6 rows and 6 columns were intensified before any was repeated. The user was requested to attend to the desired character and keep a mental count of the number of times it was intensified in order to communicate it.

In spite of being one of the most common types and also one of the most studied $\mathrm{BCls}$, it was not until recently that studies presented changes to the original design and paradigm used in the "P300 Speller" (Jin et al. 2012; Kaufmann et al. 2011; Townsend et al. 2010; Townsend and Platsko 2016).

A P300-based $\mathrm{BCl}$ has an apparent advantage in that it requires no initial user training: P300 is a typical, or "naïve", response to a desired choice (Donchin, Spencer and Wijesinghe 2000; Wolpaw, Birbaumer, McFarland, Pfurtscheller and Vaughan 2002). However, in the long term, P300 amplitude might habituate after 
repeated presentation of the oddball paradigm (Nicolas-Alonso and Gomez-Gil 2012; Ravden and Polich 1999).

\subsubsection{Sensorimotor cortex activity and ERD/ERS}

Movement or preparation for movement is typically accompanied by a decrease in EEG mu and beta rhythms (see ANNEX A. BRAIN RHYTHMS), particularly over contralateral sensorimotor cortex. This decrease has been labelled 'event-related desynchronization' (ERD). Its opposite, rhythm increase, or 'event-related synchronization' (ERS) occurs after movement and with relaxation (Pfurtscheller et al. 1998; Wolpaw, Birbaumer, McFarland, Pfurtscheller and Vaughan 2002).

Furthermore, and most relevant for BMl applications, ERD and ERS do not require actual movement: they occur with motor imagery (i.e. imagined movement), as well (Wolpaw, Birbaumer, McFarland, Pfurtscheller and Vaughan 2002). (Miller et al. 2010) measured the cortical activity during both motor imagery and its corresponding overt movement with electrocorticography (ECoG), which employs the clinical placement of electrode arrays on the brain surface. They concluded that imagining a movement typically produces a neural activity that is spatiotemporally similar to the activity generated by actual movement but smaller in magnitude (approximately $25 \%$ of the latter, in their experimental conditions). However, when participants used motor imagery to control a computer cursor and received feedback, the cortical activity was increased and even exceeded that of the overt movement.

One of the challenges in the development of an effective sensorimotor rhythm based $\mathrm{BCl}$ system is to effectively discriminate amongst different motor imagery (MI) tasks. Hand areas, foot areas, and the tongue area are comparatively large and topographically different, being possible choices for MI-based BCls, but resulting in a limited number of commands (Hamedi et al. 2016; Schlögl et al. 2005; Shan et al. 2015).

Over the last two decades, the most prominent groups on $\mathrm{BCl}$ research based on sensorimotor rhythms considering different system design approaches have been the Wadsworth BCl (McFarland and Wolpaw 2011; Wolpaw et al. 2003) and the Graz $\mathrm{BCl}$ (Pfurtscheller et al. 2000; Pfurtscheller et al. 2003) groups. 
In the standard Graz BCl paradigm for the discrimination of two mental states, the experimental task is to imagine either right-hand or left-hand movement depending on a visually presented cue stimulus. Depending on the direction of an arrow presented on a screen, the participant is instructed to imagine, e.g., a movement of the left or the right hand (Neuper et al. 1999). Over the years, additional movements have been added (e.g. both feet together) to the paradigm (Neuper et al. 2009).

\subsection{PERFORMANCE MEASURES}

A problem that is not completely solved yet in the literature is how to define a performance index that can be used as standard to compare different systems. Despite the fact that this issue has been identified several years ago and, since then, solutions have been proposed, the lack of a common performance rate in the studies persists as an obstacle for adequate cross-comparisons. In addition to that, studies vary in sample sizes and trial numbers and attention to statistical significance must be taken.

The information transfer rate (ITR), or bit rate (in bits/trial or bits/min), previously defined in equation (1), is a widely applied metric to assess overall performance of BMIs, but this is valid under strict assumptions that are sometimes ignored in literature. This metric is mostly adequate for synchronous, discrete BMls and the preconditions of using equation (1) are summarized as follows (Yuan et al. 2013):

a) BMI systems are memory-less and stable discrete transmission channels;

b) All the output commands are equally likely to be selected;

c) The classification accuracy is the same for all the target symbols;

d) The classification error is equally distributed among all the remaining symbols.

The preconditions above suggest that an output should not e related to previous inputs and outputs, errors should not be corrected and the system should not consider an idle state (because the probability of selecting an idle state is not the same as selecting other choices). In practice, several BMI systems violate one or more these assumptions (notably those of uniform selection probability and uniform classification error distribution) and researchers are encouraged to be careful in reporting ITR, especially when using ITR for comparisons between different BMI 
systems (Thompson, Quitadamo, Mainardi, Laghari, Gao, Kindermans, Simeral, Fazel-Rezai, Matteucci, Falk, Bianchi, Chestek and Huggins 2014).

Together with ITR, another of the most commonly reported metrics is the classification accuracy rate (also referred to as the true positive rate or sensitivity). Complimentary, as proposed by (Abibullaev and An 2012), the analysis of the area under the receiver operating characteristic (ROC) curves, which plot the sensitivity versus 1 minus the false positive rates (specificity) (Singh and Dan 2006), can be used to evaluate the discriminatory power of the classifier.

Further, classification accuracy above $70 \%$ is often considered as the minimum performance requirement for a BMI for communication establishment and user controllability (Pfurtscheller, Allison, Brunner, Bauernfeind, Solis-Escalante, Scherer, Zander, Mueller-Putz, Neuper and Birbaumer 2010; Power, Kushki and Chau 2012). Several studies report that a non-negligible portion of users is not able to attain control of the system (approximately 15-30\%) and some groups classify this phenomenon as "BMI illiteracy" (Allison et al. 2010; Vidaurre and Blankertz 2010). Although the term is controversial (other authors prefer "BMI inefficiency"), no "universal" BMI that works for all users has been developed so far and there is little argument that the phenomenon exists as an issue.

Finally, the users are part of the BMI systems and, lately, their acceptance determines the viability of future real-life applications. Therefore, the overall user experience is encouraged to be assessed through ratings or open-ended questions (Thompson, Quitadamo, Mainardi, Laghari, Gao, Kindermans, Simeral, Fazel-Rezai, Matteucci, Falk, Bianchi, Chestek and Huggins 2014).

\subsection{REAL-LIFE APPLICATIONS VERSUS STUDIES LIMITATIONS}

Although most BMI experiments are performed in a controlled (structured) environment as a laboratory, BMls are to be taken into real-life environment, where multiple stimuli from different modalities (visual, auditory, cognitive) may occur individually or simultaneously. Besides, the movements in this real-world scenario can cause motion artifacts (Strait and Scheutz 2014a) and it may be difficult for the user to keep attention for a long time (Tomita, Vialatte, Dreyfus, Mitsukura, 
Bakardjian and Cichocki 2014). Furthermore, practical applications should be able to identify the user's mental states in order to differentiate the active and operational state (Morioka et al. 2014) from the resting one (Bogler et al. 2014; Nakao et al. 2013), when the user does not intend to operate the device.

While most experiments consider a paradigm where the stimuli onset and duration is known, in many realistic cases this is not likely to be true. Therefore, the performance and the usability of BMls that are based on event related potentials (ERPs) or on steady state visual evoked potentials (SSVEPs), for example, may not be achieved in a real-life environment. Then, evoked potentials based BMls, could send signals when the user did not intend to convey anything (false positives), which can be especially problematic during breaks or resting periods (Durantin et al. 2014; Pfurtscheller, Allison, Brunner, Bauernfeind, Solis-Escalante, Scherer, Zander, Mueller-Putz, Neuper and Birbaumer 2010).

In the real-world (unstructured environment), according to the BMI operation paradigm, a combination of sensors (e.g. cameras, light detectors, microphones) and/or brain signal acquisition techniques must be employed to detect: a) if the required perceptual resources are available for normal operation of the system; $b$ ) if unexpected noise or stimuli are providing undesired inputs to the user, in order to avoid false positives or temporarily interrupt the system operation; c) if the user intends to rest, to make a pause or if his/her attention drops, in order to switch the BMI operation to idle mode.

Further limitations that should be considered before BMls can be used in real-life are described below.

\subsubsection{Users}

As noted by (Fujiwara et al. 2004; Naseer and Hong 2013), the results from experiments performed on healthy individuals may differ from those performed on patients such as individuals suffering from tetraplegia, lateral amyotrophic sclerosis or other neuro-motor diseases that may lead to a completely paralyzed, or "locked-in" condition. These patients, despite being the target group for BMI operation, generally have lower performance rates. It is not clear if the differences between the two 
groups (healthy individuals and patients) are due to the disruption of the sensorimotor feedback loop or to motion artifacts caused by the individual performing covert or unsuppressed movements which, obviously, cannot occur with certain patients (Blokland, Spyrou, Thijssen, Eijsvogels, Colier, Floor-Westerdijk, Vlek, Bruhn and Farquhar 2014; Tehovnik and Chen 2015; Tehovnik, Woods and Slocum 2013).

Besides, the classification accuracy can vary within a group of healthy individuals under the same setup and task (Kaiser et al. 2014) due to individual anatomical differences as head shape or scalp-cortex distance (Naseer and Hong 2013), type of hair (thick and dark hair decreases the coupling between the electrodes/optodes and the skin, resulting in quality loss of the acquired data), aging effects (Amiri et al. 2014; Fabiani et al. 2014; Heilbronner and Münte 2013; Kahlaoui et al. 2012; Wilcox et al. 2012), gender (Kameyama, Fukuda, Uehara and Mikuni 2004) and even due to the user's ability to understand and to execute the task. Those variations occur in terms of amplitude, frequency and time of the signal acquired in the same channel for different users. Moreover, it has been observed that the offline implementation approach of several studies involves a channels selection step, where the most relevant channels are selected and others are excluded, and these channels may differ among the individuals within the same group in terms of quantity and location on the scalp (Chen, Ho, Shyu, Hsu, Wang and Lee 2014; Luu and Chau 2009; Tanaka and Katura 2011; Tomita, Vialatte, Dreyfus, Mitsukura, Bakardjian and Cichocki 2014).

As the differences between individuals and groups mentioned above indicate, there is not a single generic BMI that may be appropriate to every user. Thus, the classification issues and even of the BMl illiteracy cases can, to some extent, probably be corrected through adjustments in other parts of the system design.

\subsubsection{Number of control parameters}

The definition of the number of control parameters available depends on the degrees of freedom of the physical system that will be controlled by the user.

While any successful paradigm may be adequate for the control of a single parameter, this may not be true for the control of two or more parameters, since the 
brain active areas and the physiological and/or motor artifacts can overlap (Khan et al. 2014). In fact, the stimulus used in each task usually involves more than one input. For example, speech involves both cognitive and motor activities (Bauernfeind et al. 2014), visual stimuli may elicit emotional responses and emotion can interfere in the motor function.

Therefore, increasing the number of commands of a BMI is much more complex than combining different paradigms and implementing them simultaneously in the same system. The overall BMI limits related to number of commands and responses to inputs differentiability are still not clear and must be further studied. Nevertheless, the most appropriate paradigm selection heavily depends on system design specifications such as the control requirements of the external device and the abilities and/or disabilities of the final users.

\subsubsection{Portability}

To achieve higher performance, most BMls use signals from multiple channels over the scalp. However, the use of a large number of channels involves a long preparation time, results in inconvenience for the user and many channels may convey redundant and/or noisy signals (Arvaneh et al. 2011). Besides, the number of channel combination tests and the algorithm choice for classification imply in a computation time that may take up to several days (Tanaka and Katura 2011).

Thus, a balance between performance and user convenience is required, but finding the optimal number and positioning of the channels remains a challenge. Additionally, the implementation of algorithms that require low computational resources is desired for portable, online operation (Coyle, Ward and Markham 2007; Zhang et al. 2009; Zhang and Chase 2013). 


\section{SCOPE OF WORK}

Firstly, as previously stated in section 2, this study was limited to healthy participants and to non-invasive technologies. Further, it was not a clear outcome from the bibliographic review that adding the hemodynamic brain activity with fNIRS would necessarily lead to increased BMI performance rates. The hemodynamic response has an intrinsic delay and fNIRS studies report that the onset of the response lags behind the triggering of events by $1-2 \mathrm{~s}$, at least. Besides, once the comparison between studies is difficult, the quantification of the benefit of using fNIRS technology is very challenging as well.

Moreover, the inclusion of sources of information that are external to the CNS would also have its drawbacks. Eye-tracking systems, for example, could be useful for spellers, but in a scenario where the device to be controlled is moving in relation to the user's eye (e.g. the user has to watch his or her moving arm), their use would be troublesome (Müller-Putz and Pfurtscheller 2008). Cameras and sensors should be definitely useful in real-life applications for environment monitoring, but this would not be the case in this controlled experiment.

Considering all above, it was defined the strategy to study EEG separately from other technologies, limiting the scope of the experimental protocol of this research to EEG-based data acquisition. This approach allowed the necessary practical experience with EEG equipment, the identification of applicable signal processing strategies based on the selected paradigms and, ultimately, the understanding of which factors are limiting the BMI performance from the EEG technology standpoint. In the future, the possibility of adding modalities may be reassessed, according to specific objectives.

Additionally, some pilot tests were performed using SSVEP, P300 and motor imagery (ERD/ERS) paradigms. It became clear that motor imagery differs in its nature from the other tasks and, in spite of its unarguably high potential as an endogenous task for asynchronous operation, some participants find it difficult to perform imagery and may require extensive training before they could achieve satisfactory results. On the other hand, "naïve" users without any previous training or knowledge about BMIs can use and respond to both SSVEP and P300 stimuli. 
Furthermore, while the hybrid approach using brain signals of different natures (e.g. hemodynamic and electrical) or other external sources has been excluded from the scope of this study, the combination of different brain stimuli for EEG measurement was possible. Up to date, few studies investigated hybrid BMls based on the combination of SSVEP and P300 stimuli. Some possible benefits of a hybrid approach would be to increase the number of choices, to increase the performance rate and/or to avoid the BMI illiteracy phenomenon, but concerns about interference between the brain responses and user's comfort exist. (Z. Allison et al. 2014) reported that the hybrid P300-SSVEP condition they used improved performance with respect to the SSVEP but not to the P300 and no significant differences were found with respect to user's fatigue. (Combaz and Van Hulle 2015) concluded that it is possible to detect both P300 and SSVEP simultaneously and that it could provide an advantage over pure P300- and SSVEP-based ones in terms of communication rates. (Wang et al. 2015) developed a new shape changing instead of color changing hybrid paradigm that achieved a classification rate that was as high as the pure SSVEP paradigm and the P300 paradigm, suggesting that it could be used to expand the group of $\mathrm{BCl}$ users. However, these groups used different hybrid paradigms. For example, the goal of the design used by (Wang, Daly, Allison, Jin, Zhang, Chen and Wang 2015) was to reduce illiteracy and could not be used to expand the number of possible choices. Here, we hypothesize that the strongest responses to each stimulus (SSVEP and P300) would occur in different areas over the scalp and we designed a novel hybrid paradigm based on simultaneous SSVEP and P300 stimulation that could be used to increase the performance rate, reduce illiteracy and/or increase the number of choices to complete the comparison between the brain stimulation methods.

In short, the scope of this study was limited to experimental paradigms based on three tasks that did not require neurofeedback training (SSVEP, P300 and hybrid of SSVEP and P300) for the offline operation of a discrete synchronous EEG-based BMI that allowed four choices to the user. 


\section{OBJECTIVES}

The specific objectives of this research project were:

1) To systematically compare three brain stimulation methods and different signal processing techniques and select the best method for eventual development of a discrete brain-machine interface that allows the (future) translation of brain activity signals into two binary control parameters (angular speed and position) for a single joint robotic arm. The methods and techniques were compared on the following parameters:
a) Accuracy;
b) Information transfer rate (ITR);
c) Number of illiterates;
d) Individual preferences (based on questionnaire).

2) To select the best sets of 8,16 and 32 EEG channels out of 62 channels $^{3}$ in terms of accuracy, for portability increase and cost/setup time reduction purposes, evaluating the accuracy as a function of the number of channels.

${ }^{3}$ Two mastoid channels (M1 and M2) out of the 64-channel EEG headcap are required for re-referencing (data pre-processing). 


\section{METHODS}

The following subsections detail the BMI design, the three experimental paradigms considered in the protocol, the data processing techniques, the feature extraction algorithms and the performance measures for comparison that were used in this study.

\subsection{BMI DESIGN}

The BMI design for the current study was based on allowing to the user one choice out of four possibilities. The four choices result from the combination of two binary choices for each control parameter that would be implemented in the future $\mathrm{BMI}$, namely: a) clockwise (CW) or counter clockwise (CCW) direction for angular position control and $b$ ) fast or slow for speed control.

The following stages will be implemented in the future BMI (see Figure 5):

a) Brain stimulation: the brain is endogenously or exogenously stimulated;

b) Signal acquisition: signals from the brain are recorded using EEG equipment;

c) Feature extraction: signal processing techniques are used for event detection;

d) Feature translation: a transfer function converts the extracted information into control commands for the exoskeleton.

In the current study, only stages A, B and C were implemented.

A. BRAIN STIMULUS

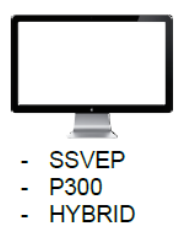

B. SIGNAL ACQUISITION

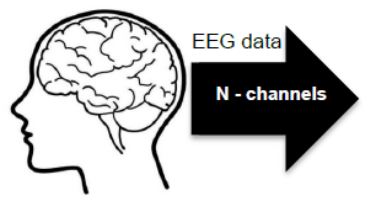

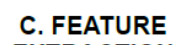
EXTRACTION

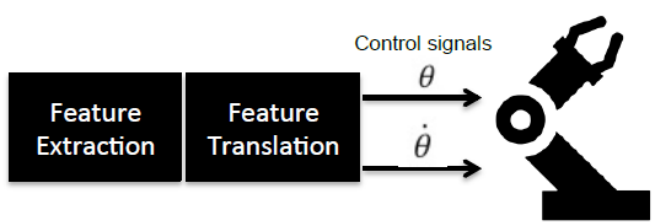

Figure 5 - General representation of a BMI design and its stages: a) Brain stimulation, b) Signal acquisition, c) Feature extraction and d) Feature translation. 
The pre-conditions for ITR calculation according to equation (1) (see section 2.3) were observed. The system was memory-less, there was no idle state, all choices were indicated the equally and the operation was synchronous. Further, there were no error correction or output prediction algorithms.

During the experiments, brain activity was registered using high density 64channel EEG integrated in a head cap. The participant was seated in front of an LCD screen placed approximately $60 \mathrm{~cm}$ away and was instructed to avoid excessive head, jaw and tongue movements to limit artifacts. After experimental setup, the participant was requested to perform three tasks based on different stimulation paradigms:

- Paradigm I: Steady State Visual Evoked Potential (SSVEP) task;

- Paradigm II: P300 task;

- Paradigm III: Hybrid task (SSVEP + P300 tasks, simultaneously).

The three tasks had six blocks with a total duration of 48 seconds each. One block consisted of one trial (40 seconds) in between two pauses (4 seconds each). During the pauses, a fixation cross was presented on the screen. During the trials, the stimuli were presented simultaneously on four different locations on the screen. A location corresponds to one of the four possible user's choices. The trials were further divided into four subtrials of 10 seconds, in which a cue (yellow square frame around a stimulus) indicated the location that the participant should pay attention to. During one trial, all of the four locations were indicated in a pseudorandom sequence, with the purpose of avoiding predictability and habituation. The same six sequences were used in the blocks of all tasks and in a pseudorandom order as well. Moreover, no sequence was repeated within the six blocks of the same task.

After the experiment, the EEG headcap was removed. Finally, information about personal preferences was collected using questionnaires.

We wrote our experimental paradigms in MATLAB (2007, The Mathworks Inc., USA) with the toolbox Psychophysics Toolbox extension (Brainard 1997; Kleiner et al. 2007; Pelli 1997) (see ANNEX A). The experimental paradigms are explained in details below. 


\subsubsection{Paradigm I: SSVEP task}

During task execution, participants sat on a chair, looking at the LCD screen.

A graphic user interface (GUI) with four virtual buttons (VB) was presented on the LCD screen (see Figure 6). A VB is a small area of the screen $(5.0$ by $5.0 \mathrm{~cm}$ ) that is similar to an icon and can have a control action associated with it. The luminance of the VBs was modulated at different blinking frequencies to produce the visual stimulation that would evoke the SSVEPs. Four different frequencies were selected from the $6-18 \mathrm{~Hz}$ range for visual stimulation according to the recommendations in the literature (Gao, Xu, Cheng and Gao 2003; Kuś, Duszyk, Milanowski, Łabęcki, Bierzyńska, Radzikowska, Michalska, Zygierewicz, Suffczyński and Durka 2013; Volosyak 2011) while taking into account the LCD refresh rate specification $(75 \mathrm{~Hz})$. Additionally, one fundamental frequency and its second harmonic should not be multiples of any other selected frequency. This resulted in the following frequencies being selected: $f 1=15(75 / 5) \mathrm{Hz}, \mathrm{f} 2=12,5(75 / 6) \mathrm{Hz}$, $\mathrm{f} 3=10,71(75 / 7) \mathrm{Hz}$ and f4=8,33 (75/9) Hz.

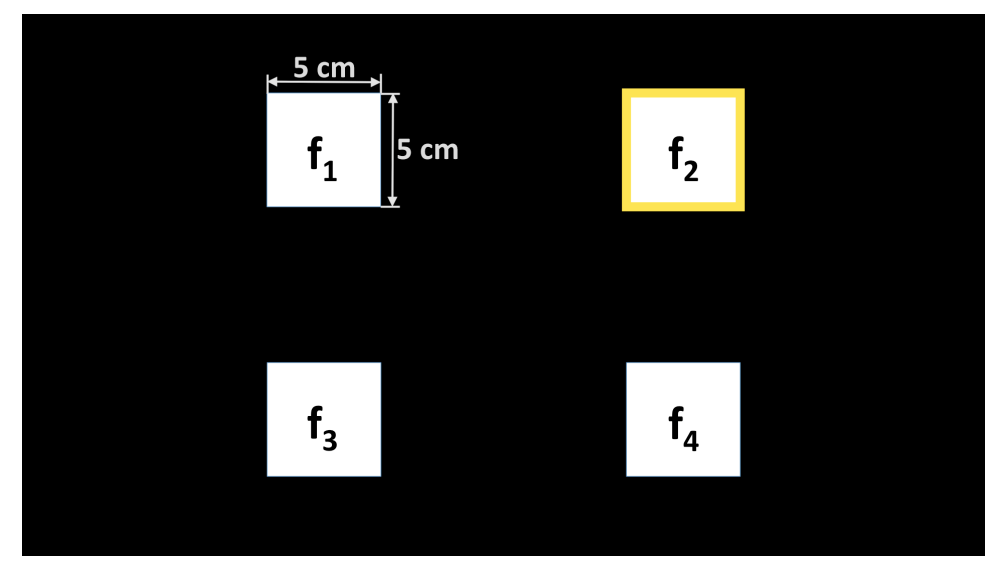

Figure 6 - GUI with four virtual buttons (VBs) and their associated flickering frequencies: $\mathrm{f} 1=15(75 / 5) \mathrm{Hz}, \mathrm{f} 2=12,5(75 / 6) \mathrm{Hz}, \mathrm{f} 3=10,71(75 / 7) \mathrm{Hz}$ and f4=8,33 (75/9) Hz. The VB with frequency $f 2$ is being indicated by its associated cue. 


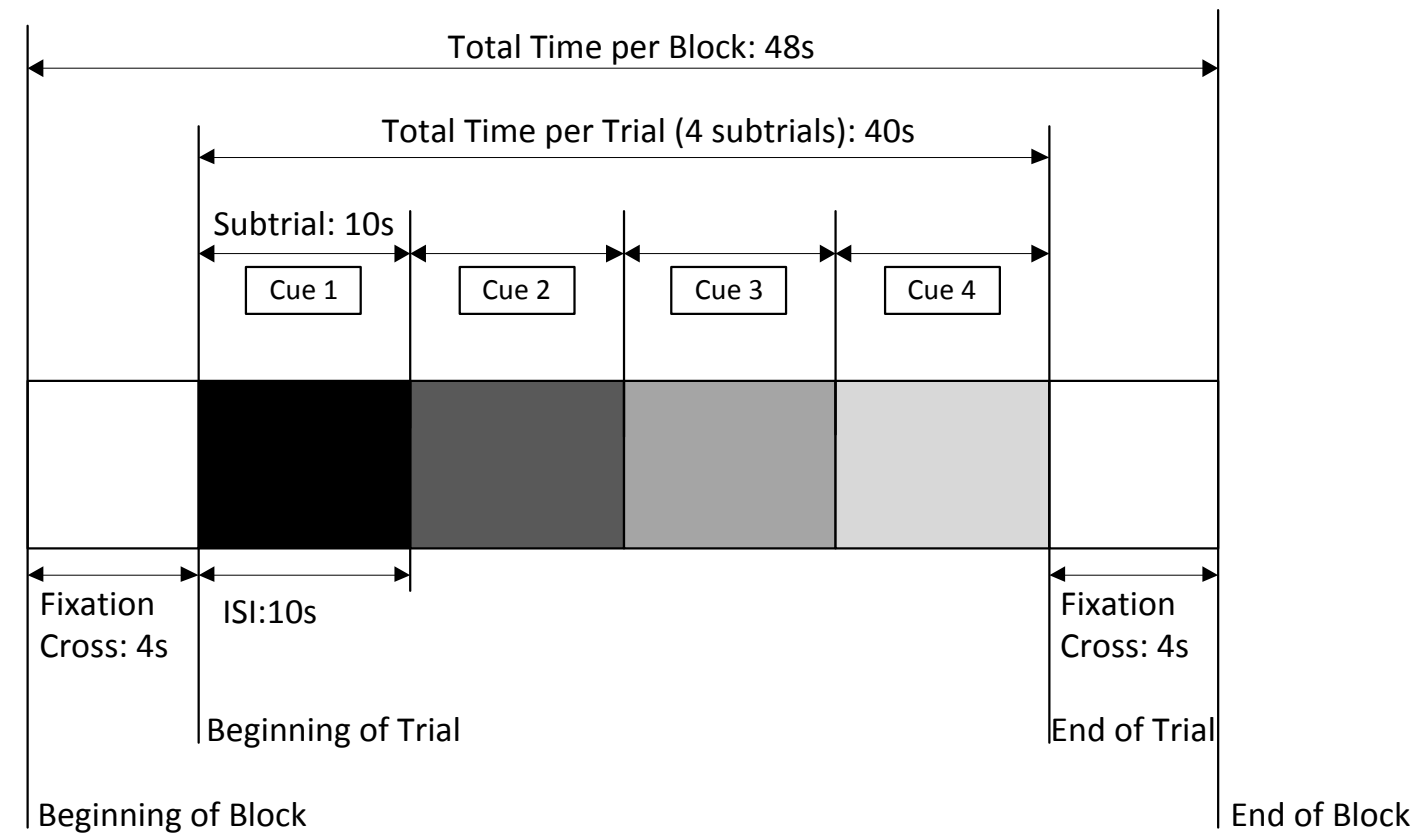

Figure 7 - SSVEP task: diagram presenting one block that consists of two pauses (initial and final) and one trial (further subdivided into four subtrials). Each subtrial is associated with a visual cue in one of the four possible locations for the user choice.

In the SSVEP task, four VBs with different blinking frequencies were displayed on the LCD screen at the same time. The task was to choose one VB out of the four possibilities on the screen. The participant chose the desired VB by visually focusing on it. A visual cue (yellow square frame around the VB) on the display indicated which VB the user should choose. One instruction block was performed to explain the task and the participant was requested to further attend to six blocks. The data from this task were used to evaluate the time needed to detect that the choice was made as a function of accuracy. Each of the six blocks contained the following elements and task instructions:

Block (see Figure 7 for reference):

1. Initial pause (4 seconds): a fixation cross was presented on the screen.

2. Trial (40 seconds): four VBs blinking at different frequencies were presented on the screen, while cues were presented. Focus on a specific VB was indicated by the associated cue for 10 seconds, until the next cue was presented. At that point, visual focus had to be switched according to the new cue. The sequence of the cues was pseudorandomized and, over one trial, all of the four positions were indicated.

3. Final pause (4 seconds): a fixation cross was presented on the screen. 


\section{Break (20 seconds).}

The total duration of Paradigm I - SSVEP task was approximately 7 minutes.

\subsubsection{Paradigm II: P300 task}

During task execution, participants sat on a chair, looking at the LCD screen.

A graphical user interface (GUI) with a 2x3 matrix of characters was presented on the LCD screen (see example in Figure 8). In a typical P300 paradigm, symbols are highlighted in a random sequence in such a manner that all symbols are highlighted before any of them is repeated. As demonstrated by (Kaufmann, Schulz, Grünzinger and Kübler 2011), superimposing the symbols of the matrix with familiar/famous faces to flash them, may increase the speed and the accuracy of P300-based BMls by eliciting other ERP components that are typically associated to processing familiar faces, particularly the N170 and N400f. Thus, the famous face of Albert Einstein was used to flash the symbols of the matrix. The user makes a choice by counting how many times the desired choice has flashed.

The main task consisted of choosing one symbol (command representation) in a 2x3 matrix with 4 possible choices plus 2 dummy symbols that were presented on a computer screen, according to Figure 8. The two dummy symbols were included because a maximum event probability of $20 \%$ is recommended for a P300 paradigm and to make the character matrix rectangular. The stimulus length (duration of a face flash) was set to $133,33(10 / 75) \mathrm{ms}$ and the interstimulus interval (ISI) was 186,67 $(14 / 75) \mathrm{ms}$. The symbols were flashed by superimposing an image with the familiar face of Albert Einstein in a pseudorandom sequence that was generated in such a manner that: a) all of the six symbols were highlighted before any of them was repeated, b) in a new sequence, one symbol from the previous sequence would not be repeated before at least two different ones have been highlighted. Thus, the minimum interval between two consecutive flash onsets in the same location (targetto-target interval) was $560 \mathrm{~ms}$. It was done to minimize the possibility of overlapping two consecutive ERPs without compromising the unpredictability of the events. The participant made a choice by mentally counting how many times the desired choice was flashed with the familiar face. A visual cue (yellow square frame around the 
symbol) was used to indicate which symbol should be chosen. One instruction block was performed to explain the task and the participant was requested to further attend to six blocks. The data from this task were used to evaluate the time needed to detect that the choice was made as a function of accuracy.

Block (see Figure 9 for reference):

1. Initial pause (4 seconds): the participant had to wait for the cue.

2. Trial (40 seconds): a $2 \times 3$ matrix with 4 possible choices (symbols) and 2 dummy symbols was presented on a computer screen. Focus on a specific symbol was indicated by the associated cue for 10 seconds, until the next cue was presented. At that point, visual focus had to be switched according to the new cue. While focusing on one symbol, the participant should mentally count how many times it was flashed with the face of Albert Einstein.

3. Final pause (4 seconds): a fixation cross was presented on the screen.

4. 20 seconds break.

5. Repeat steps $1-4$ (total of 6 blocks).

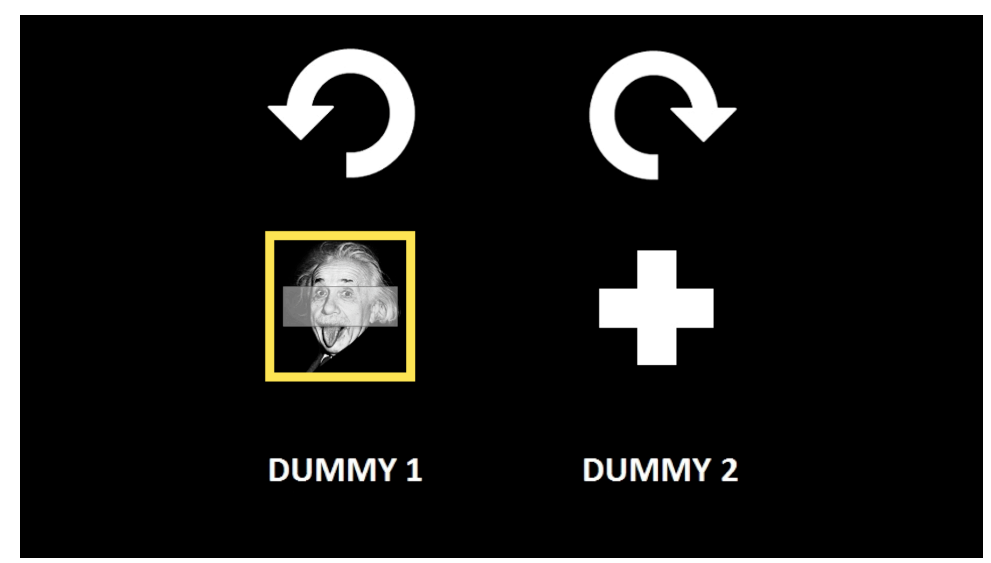

Figure 8 - Design of a 2x3 matrix with 4 possible choices (symbols) and 2 dummy symbols for P300-BCl interface (adapted from (Halder et al. 2015)). One of the symbols is being indicated by its associated cue and flashed with the famous face of Albert Einstein. 


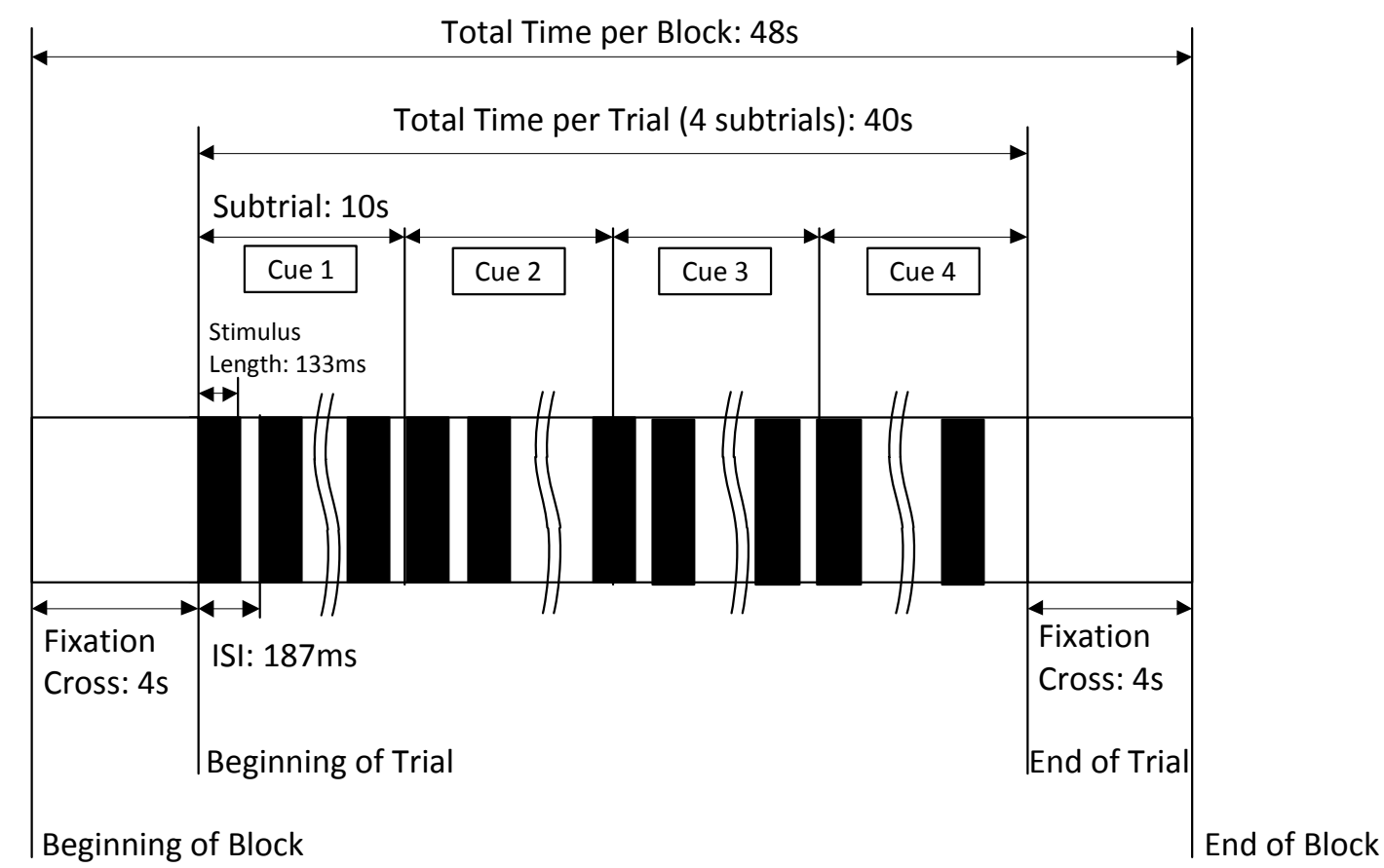

Figure 9 - P300 task diagram presenting one block that consists of two pauses (initial and final) and one trial (further subdivided into four subtrials). Each subtrial is associated to a visual cue in one of the four possible locations for the user choice.

The total duration of Paradigm II - P300 task was approximately 7 minutes.

\subsubsection{Paradigm III: Hybrid task}

In this task, a combination of the SSVEP and P300 tasks was performed. The P300 matrix was similar to the former one and two VBs from the SSVEP protocol $\mathrm{f} 2=12,5(75 / 6) \mathrm{Hz}, \mathrm{f} 3=10,71(75 / 7) \mathrm{Hz}$ were inserted in the matrix (see Figure 10). The first VB with frequency f2 was inserted in between the two upper symbols of the P300 matrix and the latter VB was inserted in between the intermediate symbols of the former P300 matrix. For technical reasons, the two dummy VBs were considered in the flashing sequences, but were not made visible on the screen. One choice was defined by the combination of one VB and one of the 2 symbols of the P300 matrix next to it, resulting in four possible choices. The participant made the desired choice by simultaneously focusing on a VB and mentally counting how many times its associated symbol was flashed with the familiar face. Two simultaneous visual cues (one yellow square frame around the symbol and another similar frame around the 
VB) were used to indicate which choice should be made (i.e. paid attention to). One instruction block was performed to explain the task and the participant was requested to further attend to six blocks. The acquired data was used to evaluate if a hybrid approach can lead to increased accuracy and performance.

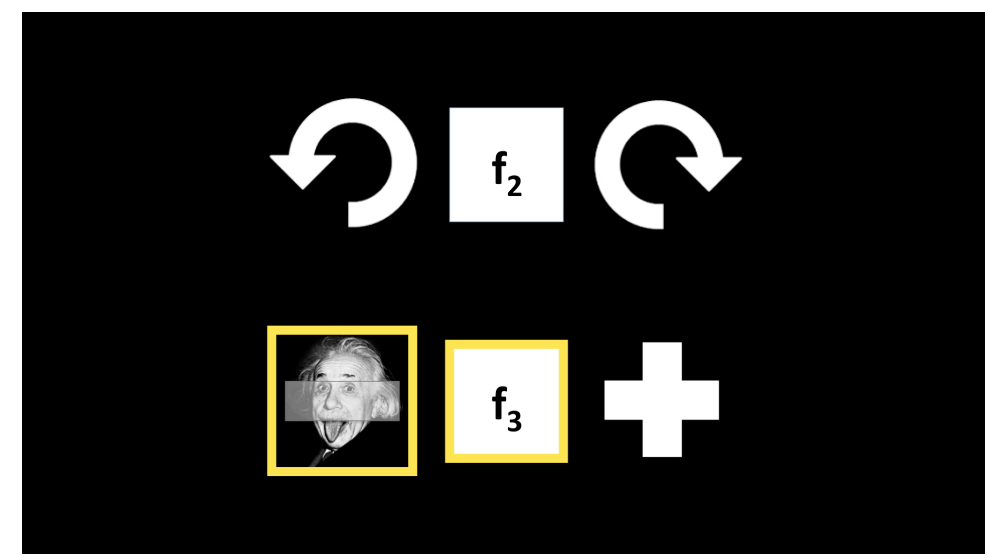

Figure 10 - GUI with two virtual buttons (VBs) and their associated flickering frequencies: f2 $=12,5(75 / 6) \mathrm{Hz}, \mathrm{f} 3=10,71(75 / 7) \mathrm{Hz} 4$ and possible choices (symbols). Each choice requires the simultaneous mental counting of how many times the famous face of Albert Einstein is flashed on it and the visual focus on the closest VB. One of the symbols and its closest VB (with frequency f3) is being indicated by its associated cues.

The total duration of Paradigm III - Hybrid task was approximately 7 minutes.

\subsection{PARTICIPANTS}

Twenty healthy adult volunteers (12 males, 8 females) with normal or corrected to normal vision were included in this study. Exclusion criteria were any history of neurological or motor disorders and use of medication influencing the central nervous system. Their median age was 27 (range 20 to 43) years. Data from one participant were excluded due to technical issues. All participants gave their signed informed consent. The experimental protocol was approved by the medical-ethical committee of the University Medical Center Groningen and the study was executed according to the Declaration of Helsinki (2013). 


\subsection{DATA ACQUISITION AND PROCESSING}

The 64-channel EEG headcap was placed according to the 10-20 international system and $\mathrm{Cz}$ positioning was checked with a metric tape. All of the headcap electrodes were filled with conductive gel using a blunt needle, after scrubbing the skin underneath using a cotton bud. The ground electrode was placed on the sternum, after cleaning the skin with scrub gel and cotton pads. The EEG signals were recorded from the scalp using a common average reference. Sampling frequency was set at $2048 \mathrm{~Hz}$. Cognitrace 3.3 (eemagine Medical Imaging Solutions $\mathrm{GmbH}$, Berlin, Germany) was used for data acquisition. Pre-processing of data was performed off-line using Brain Vision Analyzer 2.1 (Brain Products $\mathrm{GmbH}$, Munich, Germany). The data were downsampled to $256 \mathrm{~Hz}$ and bandpass filtered (0,02 - 35 $\mathrm{Hz}$, 8th order Butterworth), similar to previous studies on P300 based BMls (Donchin, Spencer and Wijesinghe 2000). This bandpass filter was also adequate for the SSVEP data processing, as the highest frequency required for analysis was $30 \mathrm{~Hz}$ (the second harmonic of $15 \mathrm{~Hz}$ ). Therefore, the same filter setting could be used for all of the tasks including the Hybrid one, simplifying the data processing. No further artefact rejection was applied and all trials of all participants were used in the analysis, in order to: a) simulate the conditions of a real-time application, b) minimize the pre-processing time and c) facilitate the comparison between different tasks.

For each task, the participant's choice was identified through its respective processing protocol and feature detection algorithm, as described below.

\subsubsection{SSVEP}

The data that were acquired during stimulation were segmented four times in Brain Vision Analyzer 2.1, using different segment lengths: $10 \mathrm{~s}, 5 \mathrm{~s}, 2 \mathrm{~s}$ and $1 \mathrm{~s}$. Ten seconds was the total stimulation time of one subtrial and the shorter lengths were selected in a manner that no segment would contain data from two different subtrials. These segment lengths were chosen to evaluate how the accuracy varies with segment length reduction, which is desired for a real-time application. A fast Fourier 
transform (FFT) was performed on each segment, using all of the points available and applying a $5 \%$ length, periodic hamming window. The resolution was set to 0.1 $\mathrm{Hz}$ (zero padding was allowed, when necessary). The pre-processed data with the resulting power output (half spectrum) of each segment were exported to text files (.dat extension) for further processing in MATLAB (2015a, The Mathworks Inc., USA).

To determine which VB participants were focussing on, the spectra of the FFT power outputs were automatically inspected for amplitude peaks at the stimulation frequencies (see Figure 11). A peak detection algorithm ("findpeaks", MATLAB 2015a) was used to detect local maxima and their respective locations in the datasets. The intensity of the response for each stimulation frequency was defined as the sum of the amplitudes of its fundamental frequency and its second harmonic, being considered as the feature for target detection. If it was no possible to find a peak neither at one of the fundamental stimulation frequencies nor at their second harmonics, the highest amplitude of the four stimulation frequencies was considered for participant choice detection.

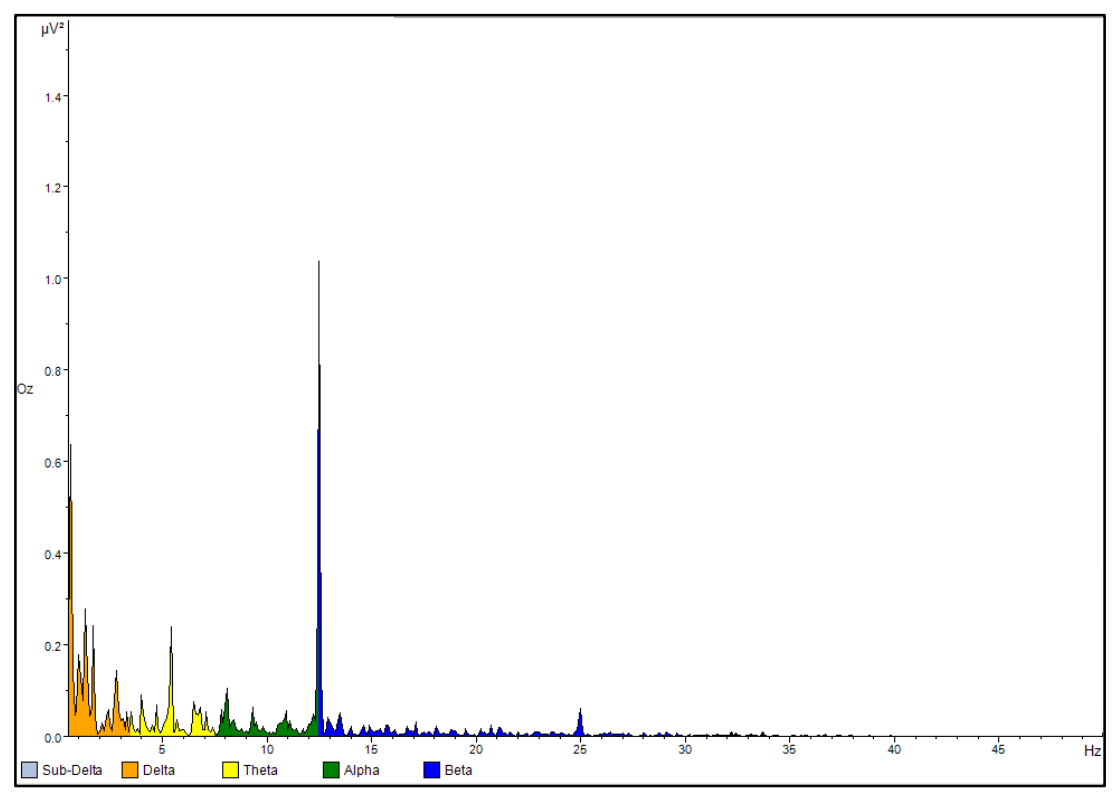

Figure 11 - Example of FFT spectrum after 10s segmentation of a subtrial where the participant had visual focus on the VB with $\mathrm{f} 2=12,5 \mathrm{~Hz}$. A higher amplitude peak is found at the fundamental frequency and a lower one at its second harmonic. 


\subsubsection{P300}

The data were first re-referenced to linked mastoids. The data acquired during stimulation trials were segmented into two different time intervals: $10 \mathrm{~s}$ and $5 \mathrm{~s}$. It is assumed that only the location containing the chosen symbol will elicit the P300. Thus, to investigate which of the four valid symbols (not dummies) of the P300 matrix the participants were attempting to choose, all of the four locations were inspected for ERPs, separately. For one given location, a flash of the familiar face on its symbol is considered a target and a flash elsewhere is considered a non-target. For each location, the data from every segment were further divided into $620 \mathrm{~ms}$ segments based on target onsets (from $-20 \mathrm{~ms}$ before to $600 \mathrm{~ms}$ after the target onset) and baseline corrected (from $-20 \mathrm{~ms}$ before the target to its onset). Since the minimum target interval was set to $560 \mathrm{~ms}$ (see Paradigm II: P300 ), some data overlap between segments could eventually occur. All of the resulting $620 \mathrm{~ms}$ segments containing the targets were averaged into one dataset for each one of the four locations, separately. The pre-processed data were exported to text files (.dat extension) for further processing in MATLAB.

Two different algorithms were used to compute the features for participant's choice detection: a) peak to peak amplitude and b) template matching.

a) Peak to peak amplitude: the difference between the lowest and the highest amplitude within the $620 \mathrm{~ms}$ segment was defined as the feature for choice detection. Two algorithms for maximum and minimum values detection ("max" and "min", MATLAB 2015a) were used to detect the maximum and the minimum values in the datasets, respectively. The dataset yielding the highest peak-to-peak amplitude amongst the four datasets was assumed to contain the ERP, with its respective location being considered as the chosen one;

b) Template match: a P300 template was built from the average of all of the attended targets (that were expected to include a P300) from all of the four locations. This template is assumed to represent the expected P300 waveform of the participant (see Figure 12). The cross-correlation between the curve of the averaged segments corresponding to each of the four possible stimulation locations and the template was taken as the feature 
for choice detection (see Figure 13). The cross-correlation algorithm "xcorr" in MATLAB was used to calculate the cross-correlation values between the datasets. The dataset from the stimulation location yielding the highest cross-correlation value amongst the four location datasets was assumed to contain the ERP, with its respective location being considered as the chosen one.

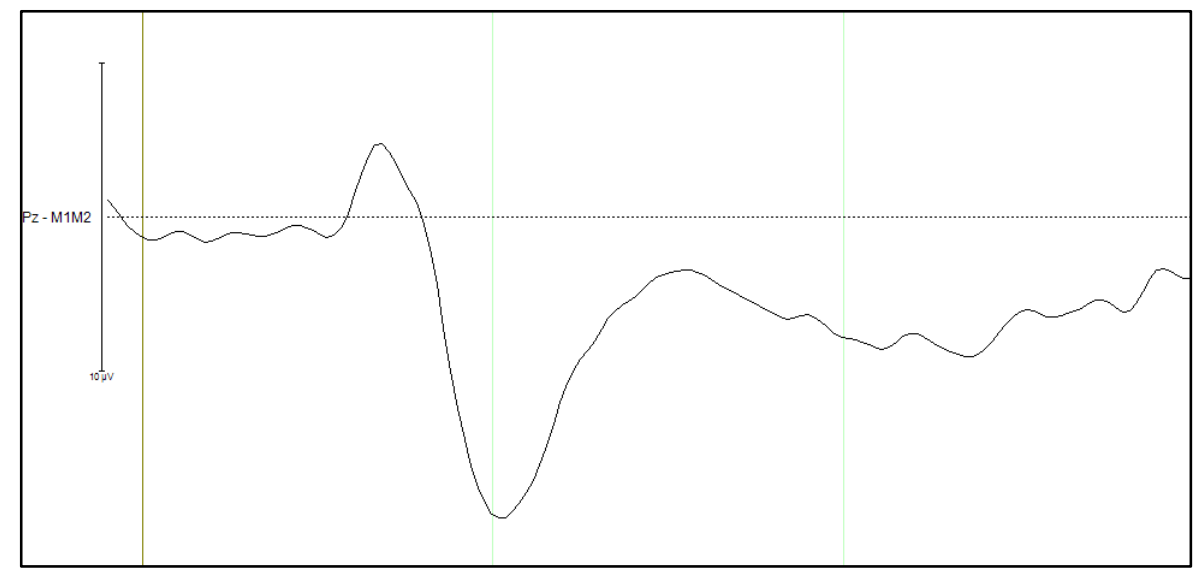

Figure 12 - Template with the average waveform of one participant for attended targets.

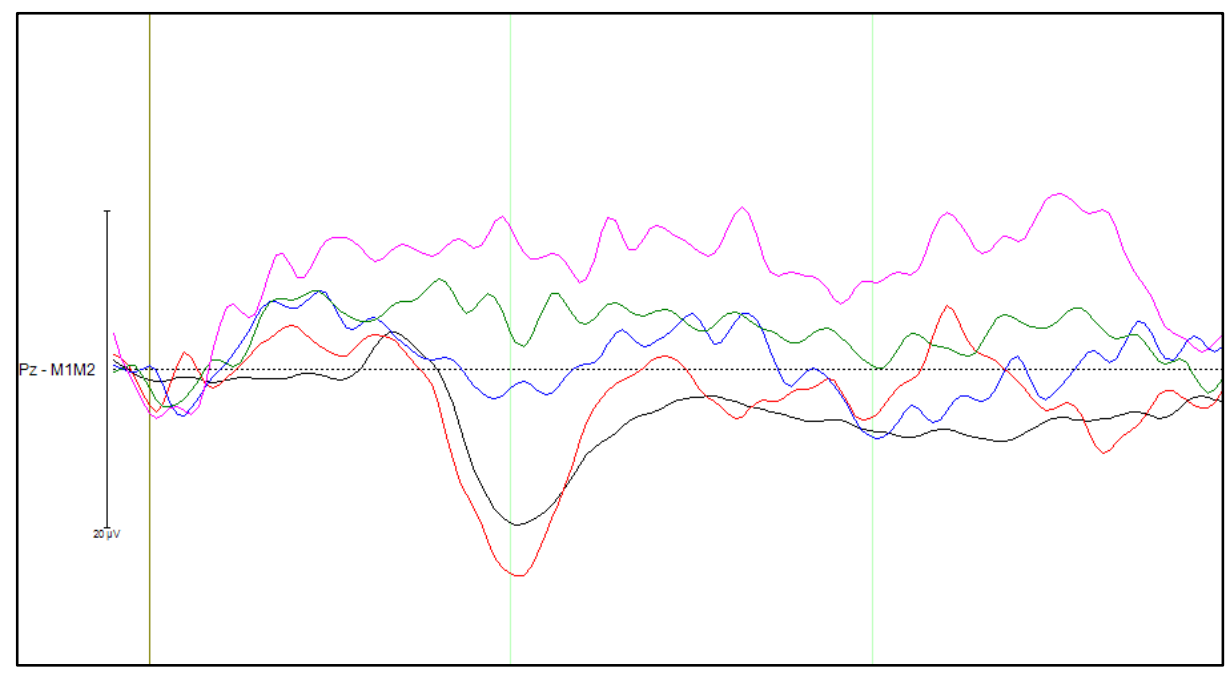

Figure 13 - Curves of the averaged segments over 10s (one subtrial) corresponding to each of the four possible locations (coloured curves) and the template (black curve), for the same participant as in Figure 11. 


\subsubsection{Hybrid SSVEP + P300}

A procedure resulting from the combination of the ones described above for the SSVEP and P300 tasks was applied to the data acquired during the Hybrid task. Some differences were taken into account:

a) Only two SSVEP stimulation frequencies were used (f2 $=12,5 \mathrm{~Hz}$ and $f 3=10,7$ $\mathrm{Hz})$;

b) Only 10 s segmentation was performed, because the results achieved were already inferior to the other tasks;

c) Only the P300 template match algorithm was used, since it gave the best results between the two algorithms that were used, and two P300 templates were created: one for each pair of symbols close to one VB. The reason for the additional template is the potential interference of the SSVEP elicited by each VB in the P300 waveforms.

Firstly, the SSVEP task algorithm was used to sort out which of the two frequencies the participant was choosing, based on the Oz channel only. After that, the template match algorithm was applied to all channels using the P300 template that corresponds to the identified frequency. Finally, the dataset from the stimulation location yielding the highest cross-correlation value amongst the four location datasets was assumed to contain the ERP, with its respective location being considered as the chosen one.

\subsection{PERFORMANCE MEASURES}

This subsection details the measures that were used for comparison of the three different experimental paradigms. 


\subsubsection{Accuracy}

Classification accuracy above $70 \%$ is often considered in the literature as the minimum performance requirement for a $\mathrm{BMI}$ for communication establishment and user controllability (Pfurtscheller, Allison, Brunner, Bauernfeind, Solis-Escalante, Scherer, Zander, Mueller-Putz, Neuper and Birbaumer 2010; Power, Kushki and Chau 2012).

This study considers the off-line operation of a synchronous BMI and the accuracy is based on the results of the analysis of data segments acquired during the stimulation trials and their comparison to the expected results according to the cues that were presented by the system during the tasks. One segment length is the minimum time needed by the system (excluding processing time) to output a response. In this study, there were 6 trials per task and each trial was further divided into subtrials where all of the 4 possible choices (locations) were equally stimulated for 10s. Thus, the maximum segmentation time that should contain only one stimulated location is 10 s, which results in 24 segments of data per task, per participant. Shorter segmentations into $5 \mathrm{~s}, 2 \mathrm{~s}$ and $1 \mathrm{~s}$ were attempted to investigate the effect of segment length (i.e. stimulation duration) on accuracy, resulting in 48 , 300 and 600 segments per task, respectively.

The accuracy $\mathrm{P}$ was calculated as a probability (fraction), for each task, as follows:

$$
P=\frac{x}{s},
$$

where $S$ is the total number of data segments and $x$ is the total number of correct segments. A segment is considered to be correct if the participant's choice that was identified by the feature detection algorithm corresponds to the same location that was being indicated by the cue in that interval. 


\subsubsection{Information transfer rate}

A standard method for BMI performance evaluation incorporating both speed and accuracy in one single value is the information transfer rate, or bit rate (in bits/trial or bits/min). It was proposed by (Wolpaw, Birbaumer, Heetderks, McFarland, Peckham, Schalk, Donchin, Quatrano, Robinson and Vaughan 2000; Wolpaw, Birbaumer, McFarland, Pfurtscheller and Vaughan 2002), and was based on previous work on Information Theory (Pierce 1980).

The bit rate $B$ (in bits/segment) was accordingly computed as:

$$
B=\log _{2} N+P \log _{2} P+(1-P) \log _{2}\left[\frac{(1-P)}{N-1}\right],
$$

where $\mathrm{N}$ is the number of possible choices and $\mathrm{P}$ is the correct choice probability (estimated accuracy). The bit rate in bits/min was computed by dividing B by the segment duration in minutes.

In this study, $\mathrm{N}=4$ and $\mathrm{P}$ was calculated as described in 5.4.1.

\subsubsection{Number of illiterates}

The number of illiterates (I) per task was determined as the total number of participants that attended to the task and did not achieve a minimum accuracy of $70 \%$ in any of the EEG channels.

\subsubsection{Individual preferences}

Each participant was asked to rank the tasks in order of preference in the individual preference questionnaire, after the tasks. We counted how many times a task was ranked at each position. The task with the highest count at the first ranking position was, therefore, considered to be the preferred one. For the remaining tasks, 
the count of the first position and second position were summed and the second place was given to the task with the highest sum.

\subsection{STATISTICAL ANALYSIS}

The EEG analysis of each paradigm resulted in measures of:
a) Accuracy;
b) Information transfer rate (ITR);
c) Number of illiterates;
d) Individual preferences (based on questionnaire)

for each of the 19 participants. First, accuracy and ITR were tested for normality using the Shapiro-Wilk test. In case of a normal distribution, accuracy was compared between paradigms using an ANOVA. Subsequently, in case of a significant difference, post-hoc testing was applied. If the data were not normally distributed, we attempted to achieve a normal distribution by appropriate transformation or using a suitable nonparametric alternative. The number of illiterates was compared between paradigms using a $\mathrm{X}^{2}$ or Fisher-exact test, depending on the number of cases per category. Individual preferences were analysed using descriptive statistics. For all tests, a significance level of $\alpha=0.05$ was assumed. All statistical calculations were performed in SPSS Statistics 24.0 (IBM Corp., Armonk, NY, U.S.A.). 


\section{$6 \quad$ RESULTS}

The following subsections present the results of the performance parameters that were evaluated in this study. Data from normally distributed variables are expressed as mean and standard deviation: mean $\pm S D$. Data from non-normally distributed variables are expressed as median and interquartile range: median (IQR). All data in figures represent mean, except for the data that are followed by an asterisk (*), which represent median.

\subsection{ACCURACY}

The results presented in this section are based on the segmentation of data acquired during the stimulation trials and their comparison to the expected results according to the cues that were presented by the system during the task. For each of the 19 participants, all data from 3 tasks (6 trials per task) were used. In this study, the number of possible choices $(N)$ was four and the theoretical chance level was $25 \%$.

\subsubsection{SSVEP task}

The data acquired during the SSVEP task ( 6 trials (24 choices) per participant, 19 participants) were segmented into segments of $10 \mathrm{~s}, 5 \mathrm{~s}, 2 \mathrm{~s}$ and $1 \mathrm{~s}$. The single channel that achieved the highest average accuracy was $\mathrm{Oz}$ for any of the four segmentation times considered (see Figure 14). The accuracies at Oz were 100\% (88-100) (10s segmentation, 456 segments), 92\% (77-100) (5s segmentation, 912 segments), $78 \pm 19 \%$ (2s segmentation, 2280 segments) and $68 \pm 20 \%$ (1s segmentation, 4560 segments). 


\section{SSVEP TASK - Accuracy (\%) per channel}

Segmentation 10s

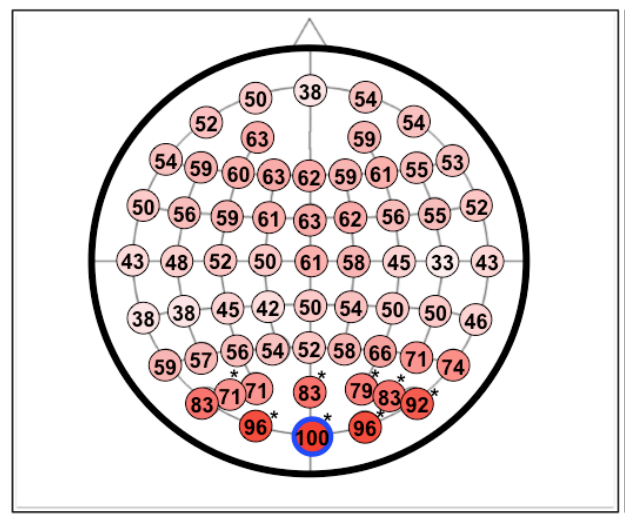

Segmentation 2s

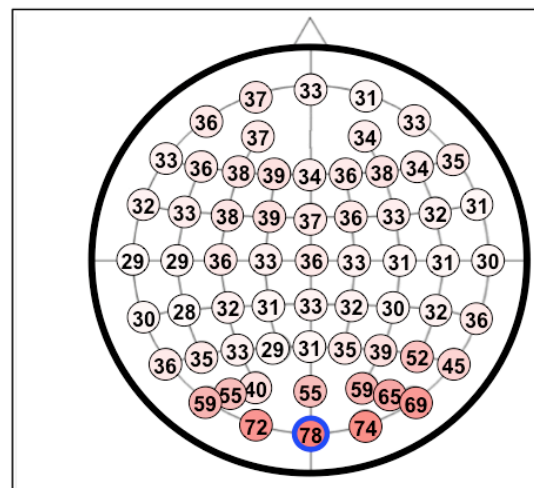

Segmentation 5s

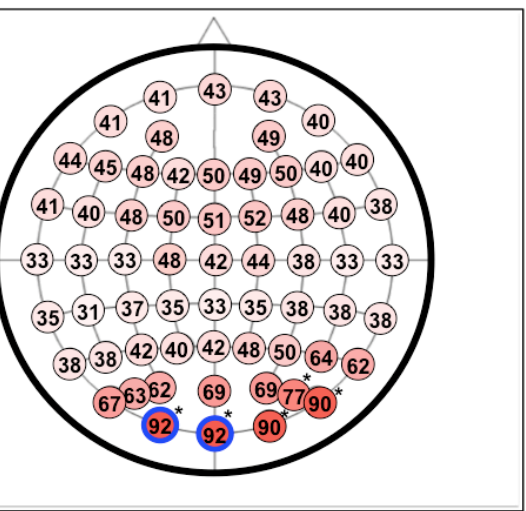

\section{Segmentation $1 \mathrm{~s}$}

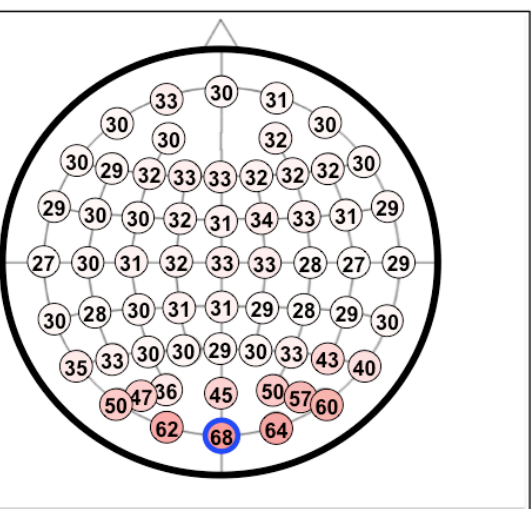

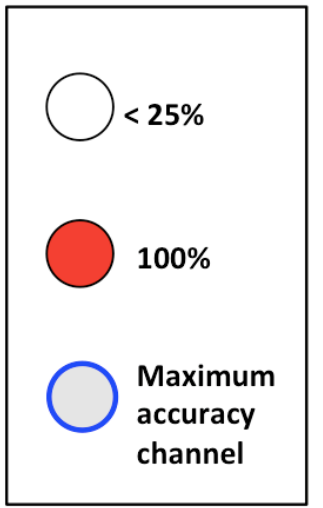

Figure 14 - SSVEP TASK - Average accuracy (\%) per channel at different segment lengths - 6 trials (24 choices) per participant, 19 participants, in red color scale. The electrode with the highest accuracy is highlighted (blue border) and the respective values are displayed inside their electrodes.

To illustrate the variability in accuracy over segment lengths between participants, a graphical overview of the results (user choice that was identified by the feature detection algorithm) from five participants, based on the $\mathrm{Oz}$ channel, in comparison to the expected results (template) is presented in Figure 15. The information corresponds to one trial where the VBs with frequencies $\mathrm{f} 1(15 \mathrm{~Hz}), \mathrm{f} 2$ $(12,5 \mathrm{~Hz}), \mathrm{f} 3(10,71 \mathrm{~Hz})$ and $\mathrm{f} 4(8,33 \mathrm{~Hz})$ were indicated, in this order. 


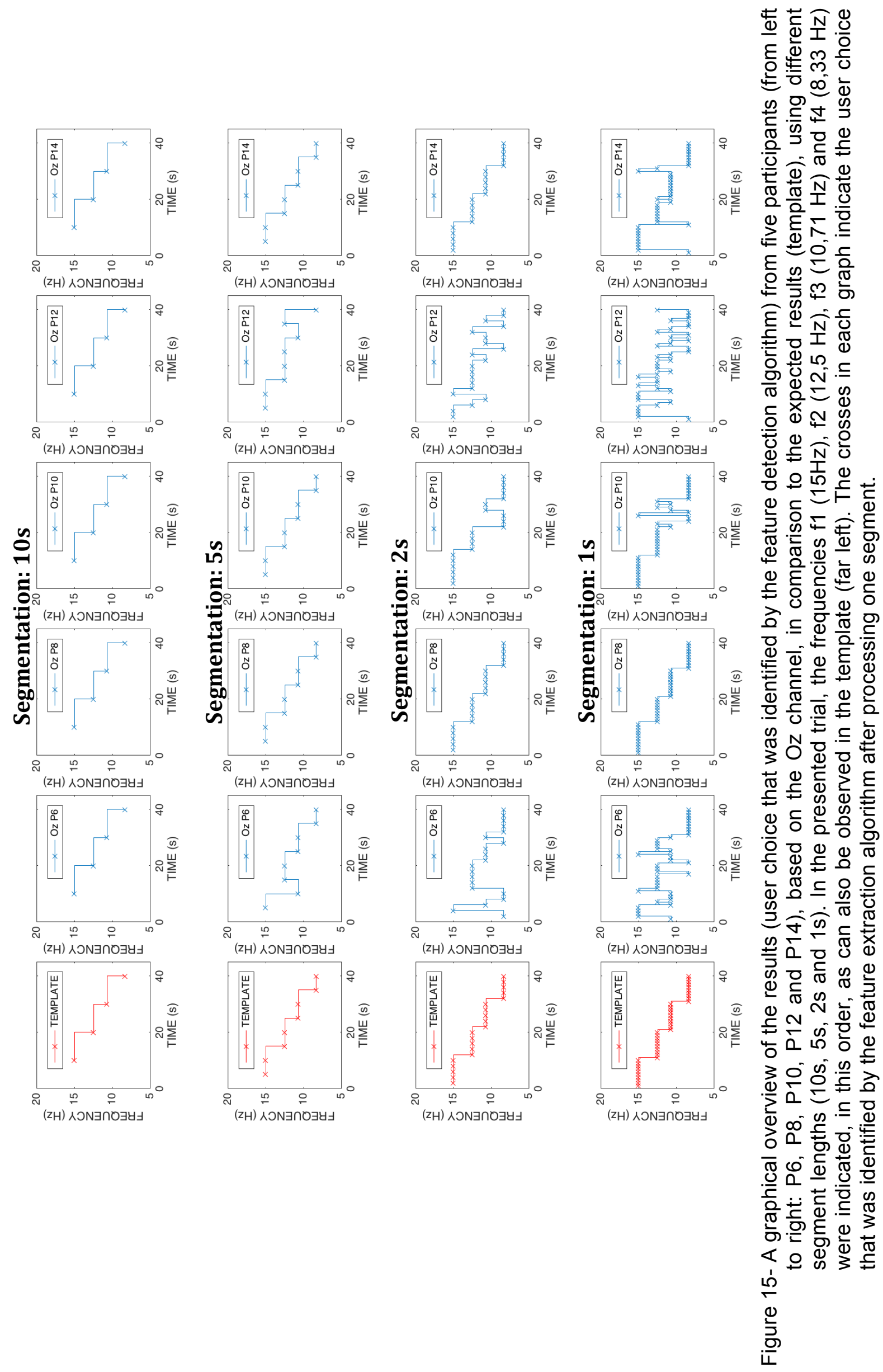


To illustrate that even though $\mathrm{Oz}$ was the best performing channel on average, there was variability in the best performing channel across participants, a footprint displaying the average accuracy per channel (10s segmentation) in a red color scale and highlighting the channel(s) that achieved the highest accuracy per participant is presented in Figure 16, for all participants. The figure also illustrates that many participants, particularly those with high all-over accuracy, have more than one EEG channel with maximum performance. 


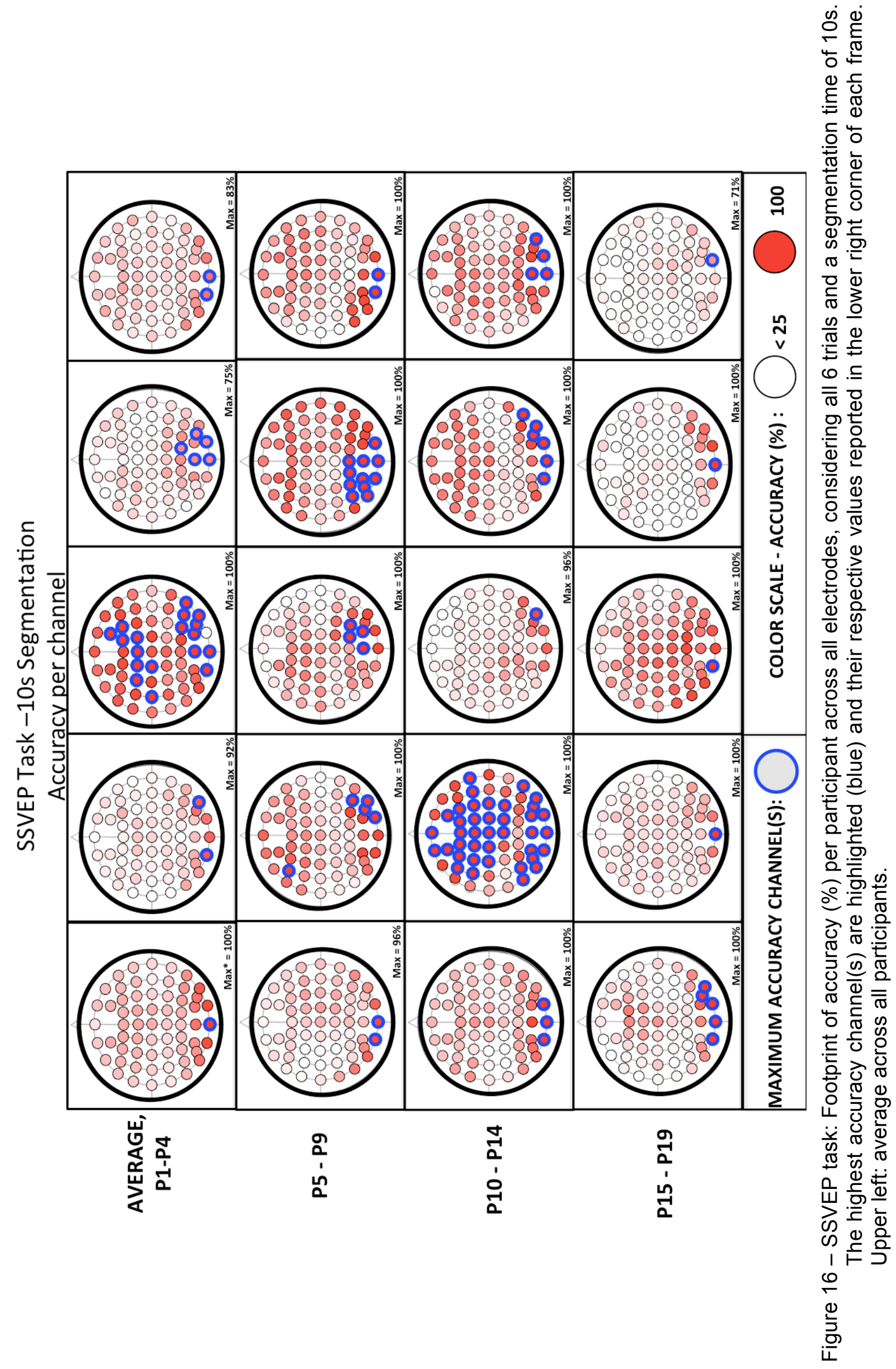


Considering the best performing channel for each participant, the average accuracy achieved was $100 \%$ (96-100), using 10s segmentation, $100 \%$ (83-100) using 5 s segmentation, $89 \%$ (70-95) using 2 s segmentation and $74 \pm 18 \%$ using 1 s segmentation (see Figure 17a). In Figure 17a, the performance for $\mathrm{Oz}$ (mentioned before) is also indicated. To illustrate the variability between participants, Figure 17b and Figure 17c display the curves from each participant that form the best channel and the $\mathrm{Oz}$ accuracy curves that were displayed in Figure 17a.

According to a Kruskal-Wallis test, there was a statistically significant difference in accuracy between the four groups with different segmentation lengths $(H(3)=25,086, p=0,000)$. Another Kruskal-Wallis test was performed on the three groups with longer segmentation lengths and also indicated a significant difference between groups $(H(2)=12,541, p=0,002)$. The Kruskal-Wallis test that was performed on the three groups with lower segmentation, indicated a significant difference between groups, as well $(H(2)=11,178, p=0,004)$. Post-hoc Mann-Whitney tests indicated that there was no significant difference for accuracy between the $5 \mathrm{~s}$ and the 2s segmentation groups $(U=110, Z=-2,092, p=0,036$, Bonferroni correction) and neither between the $5 \mathrm{~s}$ and the $10 \mathrm{~s}$ segmentation groups $(U=145, Z=-1,192$, $p=0,233)$, whereas a significant difference was found between the $10 \mathrm{~s}$ and the $2 \mathrm{~s}$ groups $(U=63, Z=-3,542, p=0.000)$. 
SSVEP TASK

ACCURACY (\%) - ALL TRIALS AVERAGE

a)

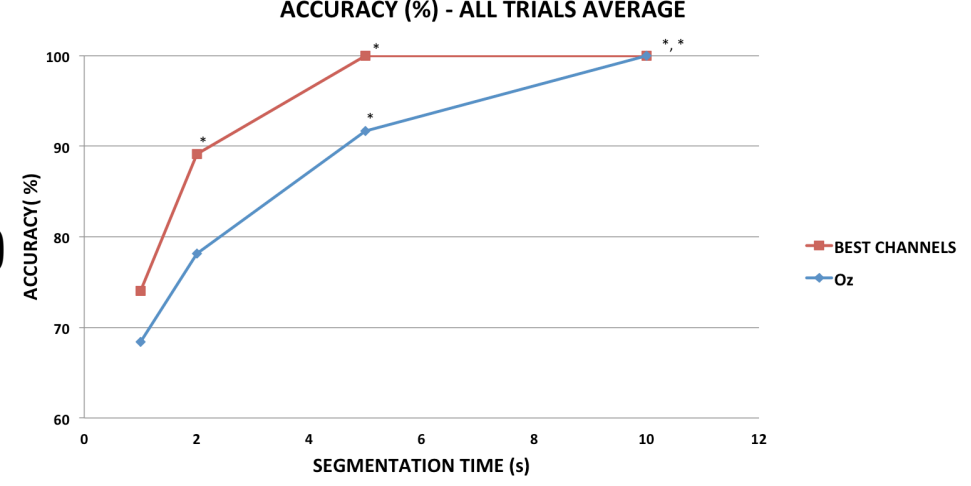

SSVEP TASK

Best Performing Channel per Participant

b)

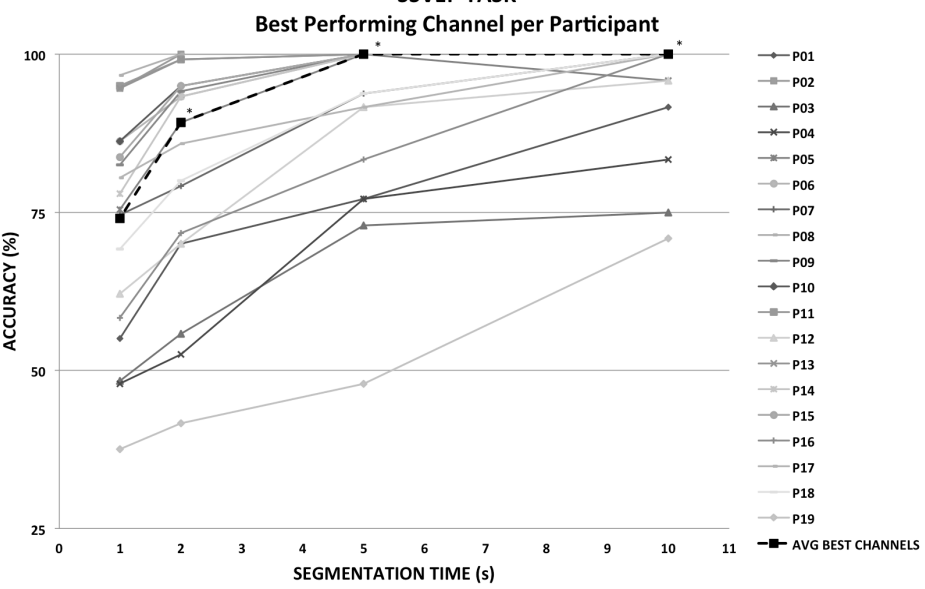

SSVEP TASK

Oz Channel per Participant

c)

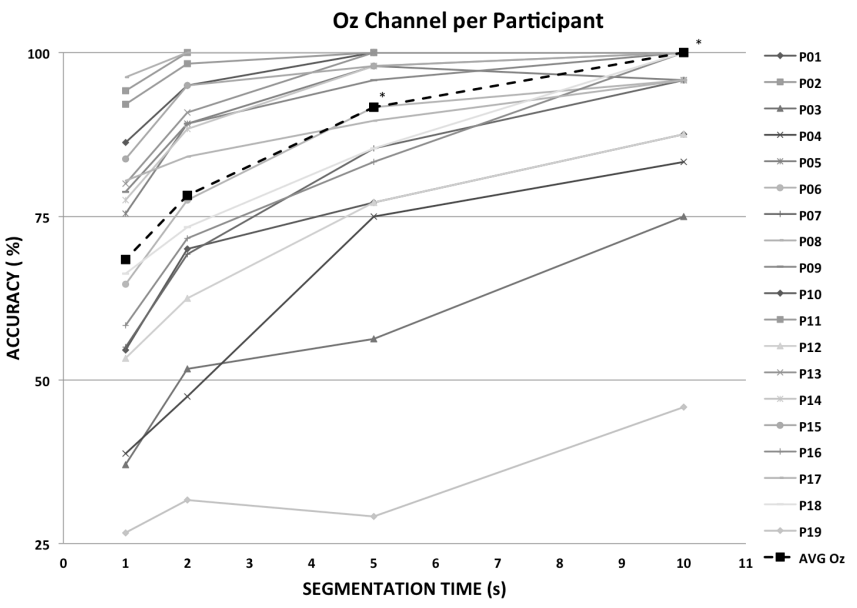

Figure 17 - SSVEP task: a) Average accuracy (\%) vs. Segmentation time (s) curves based on $\mathrm{Oz}$ channel only and on the best performing channel of each participant; b) and c) curves of each participant for both average curves presented in a). 


\subsubsection{P300 task}

The data acquired during the P300 task were segmented in 10 s and $5 \mathrm{~s}$ segments. The single channel that achieved the highest average accuracy for the 10s segmentation was Po8 for both feature extraction algorithms used. The accuracies were $82 \pm 15 \%$ and $79 \pm 13 \%$ for the "peak to peak" and "template match" algorithms, respectively. Figure 18 shows that although the "peak to peak" algorithm resulted in slightly higher accuracy than "template match" when using 10s segmentation, these results were inverted when using 5 s segmentation. In the latter case, the highest accuracies achieved for a single channel were $58 \pm 11 \%$ (Po8 channel) and $67 \pm 14 \%$ (Ft8 channel) for the "peak to peak" and "template match" algorithms, respectively. No further analyses with shorter segments were performed, as the results for 5 s segmentation were inferior compared to the SSVEP task.

P300 TASK- Accuracy (\%) per channel

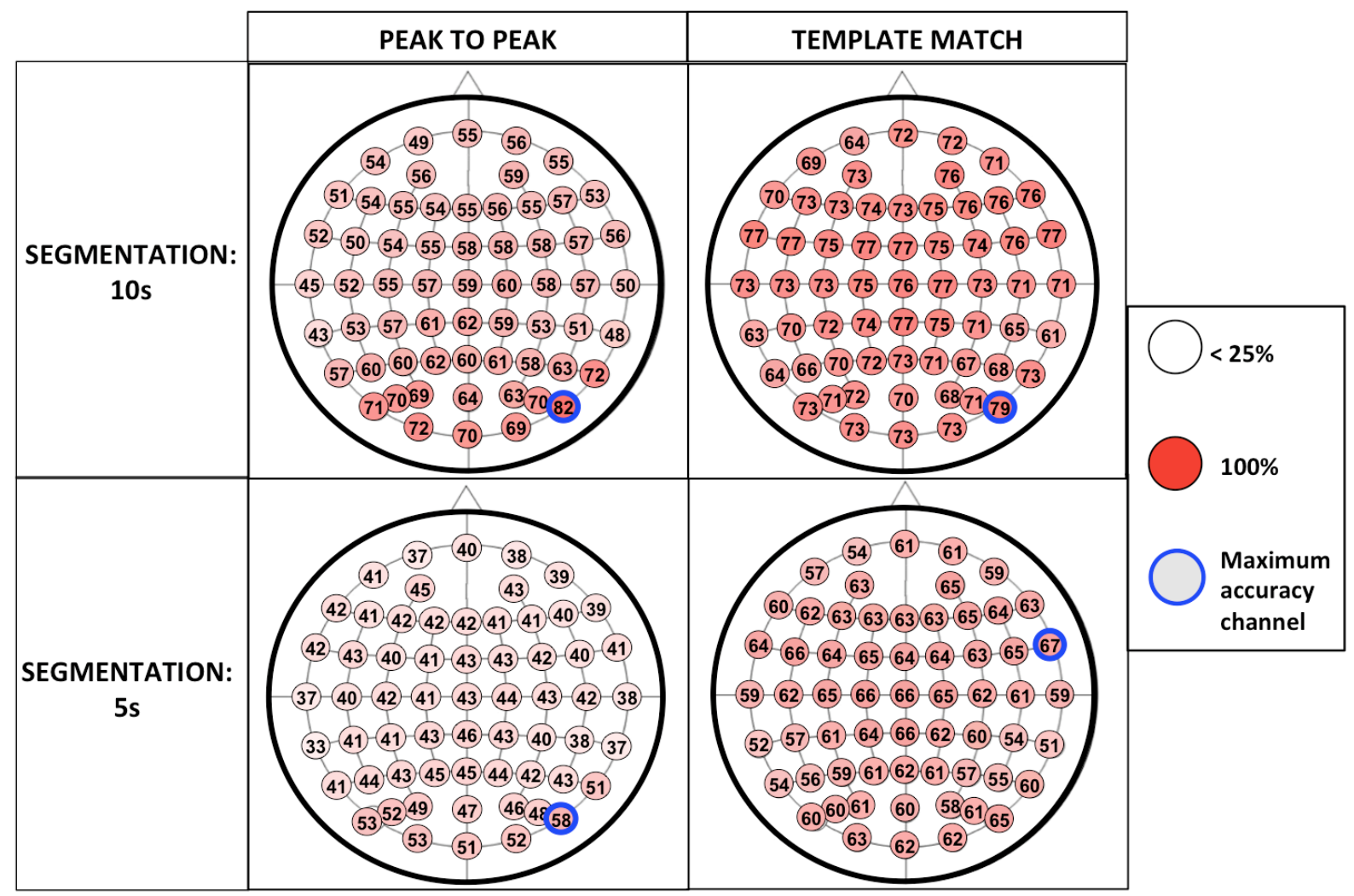

Figure 18- P300 TASK: Average accuracy (\%) per channel at different segmentation lengths - 6 trials (24 choices) per participant, 19 participants. The electrode with the highest accuracy is highlighted (blue border) and the average accuracy values are displayed in each electrode. 
To illustrate the variability in the best performing channel across participants and also in the region where it is located, a footprint displaying the accuracy per channel (10s segmentation) in a red color scale and highlighting the channel(s) that achieved the highest accuracy per participant is presented in Figure 19, for all participants. The figure also illustrates that although Po8 was the best performing channel on average, it was not the channel with maximum performance for most of the participants. 


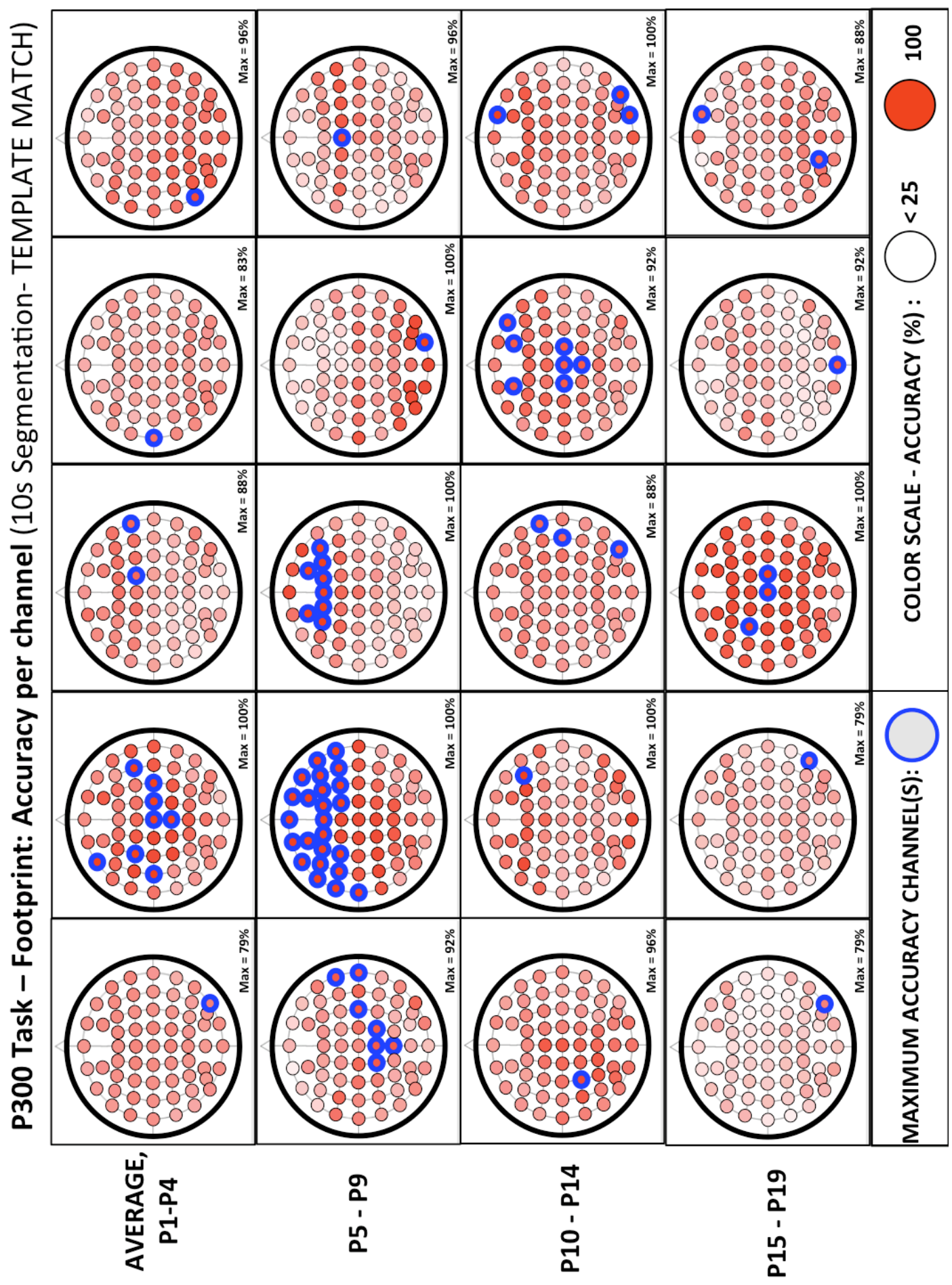

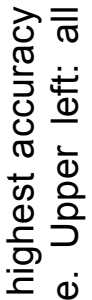

Ð

i)

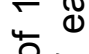

(4)

Е

으 잉

歾

는

ঠ্

으

完

of

은 응

0 웅

宁

뭉

응

등 궁

$+$

需言

음

密

(

बे

응

○ㅇำ

흉

ํํ을

ن

पั

흠

융을 용 ४ 赔 के

월

ำ

미

옥 
Figure 20 shows how both feature extraction algorithms that were used in the P300 task perform over 10 s and 5s segmentation lengths if: a) the best performing channel per participant or b) the best performing channel on average is selected.

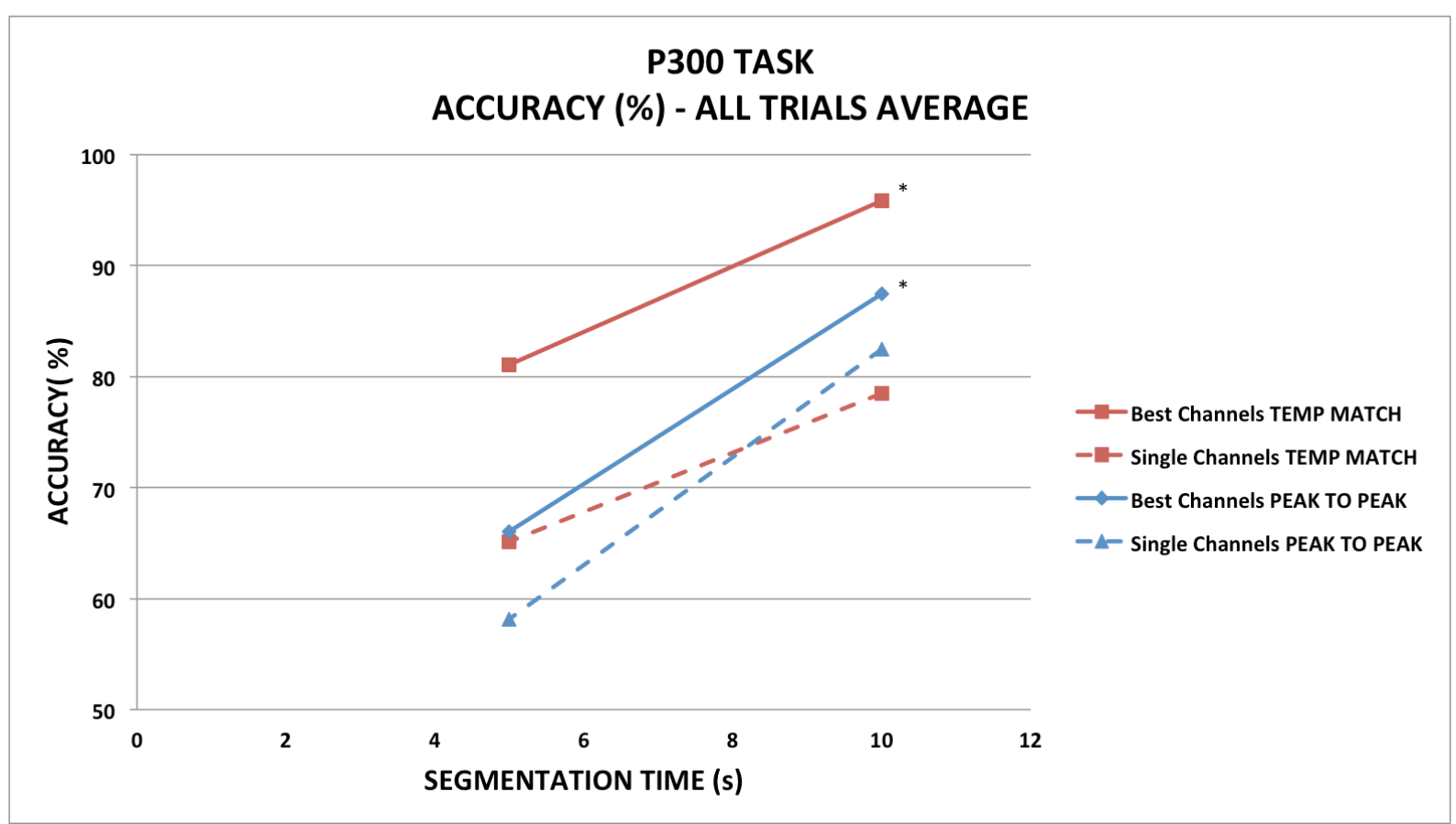

Figure 20- P300 task: Accuracy (\%) vs. Segmentation time (s) curves based on Po8 channel only and on the best performing channel of each participant, for both feature extraction algorithms "peak to peak" and "template match";

Considering the best performing channel from each participant, the median accuracy achieved using 10 s segmentation was:

a) $88 \%$ (83-100) for the "peak to peak" algorithm;

b) $96 \%$ (88-100) for the "template match" algorithm.

Considering the best performing channel from each participant, the mean accuracy achieved using 5 s segmentation was:

a) $66 \pm 10 \%$ for the "peak to peak" algorithm;

b) $81 \pm 10 \%$ for the "template match" algorithm.

Mann-Whitney tests indicated that there was no significant difference in accuracy between the "template match" and the "peak to peak" algorithms for $10 \mathrm{~s}$ segmentation $(U=149, Z=-0,948, p=0,343)$, but this difference was significant for $5 \mathrm{~s}$ segmentation $(U=51, Z=-3,805, p=0,000)$. Additionally, accuracy differed significantly between 10 s and $5 s$ segmentation for the "template match" algorithm $(U=59, Z=-$ $3,568, p=0,000)$. 
No further analyses with shorter segments were performed, as the results for $5 \mathrm{~s}$ segmentation were already significantly inferior to 10 s segmentation.

Additionally, the "peak to peak" algorithm resulted in an accuracy below $70 \%$ (minimum level required for BMI controllability) for $5 \mathrm{~s}$ segmentation which was a lower accuracy performance in comparison to the "template match" algorithm.

Figure 21 illustrates the variability between participants and that some of them performed better with the "peak to peak" algorithm, in spite of the higher accuracy results that were presented before, on average, for the "template match" algorithm. The results are presented for each participant, based on their best performing channel and 10 s segmentation.

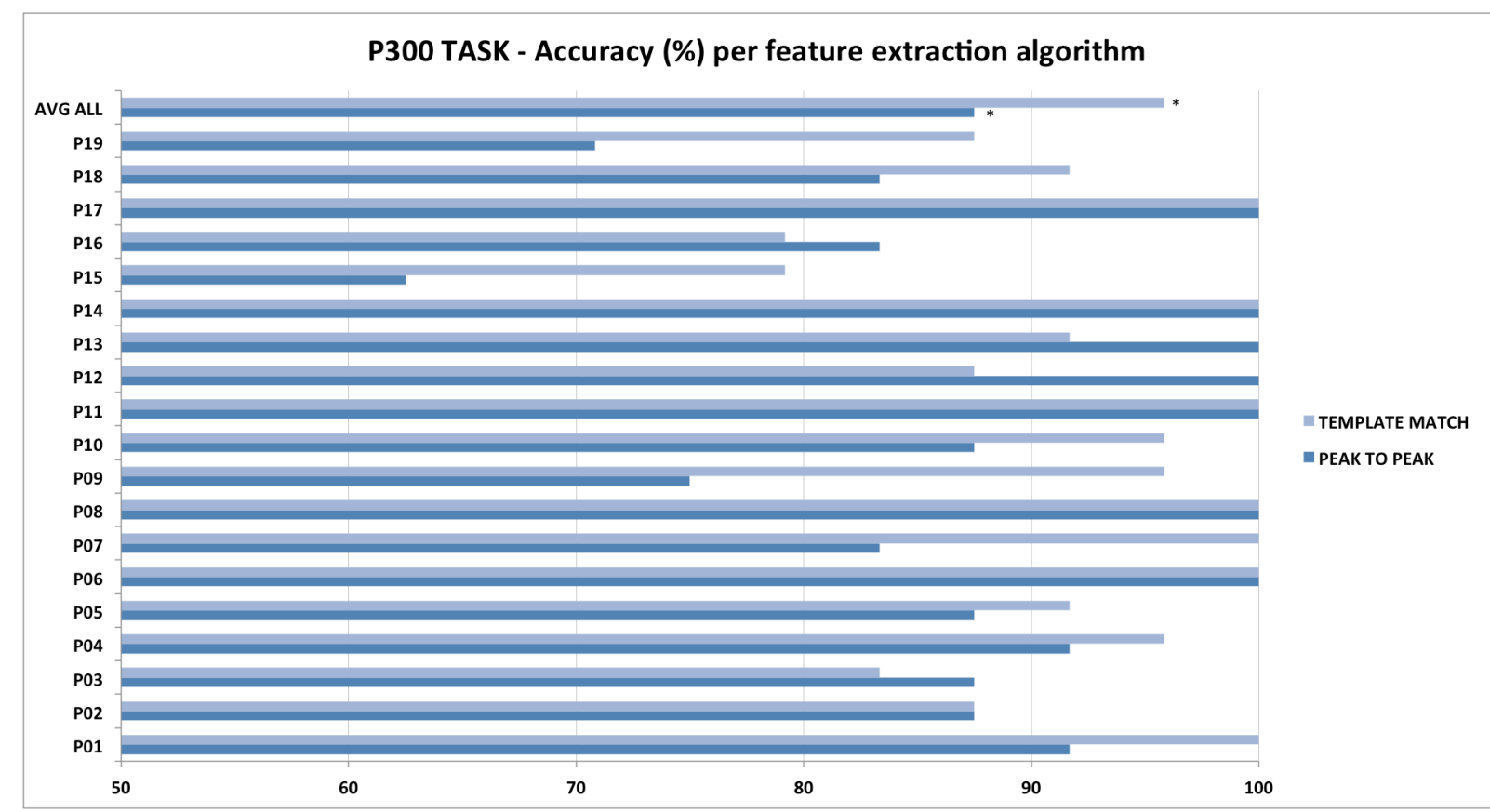

Figure 21 - P300 task: accuracy (\%) achieved by each participant based on their best performing channel and 10s segmentation, for both feature extraction algorithms.

\subsubsection{Hybrid (SSVEP + P300) task}

The data acquired during the hybrid task were segmented in 10s segments only. Both single channels Af3 and Po7 achieved the highest accuracy of $68 \pm 11 \%$ and $68 \pm 13 \%$, respectively (see Figure 22 ). If the best performing channel from each participant was considered, the median accuracy was $83 \%(75-88)$. 
No further analyses with shorter segments were performed, as the results for $10 \mathrm{~s}$ segmentation were already much inferior to those of other tasks.

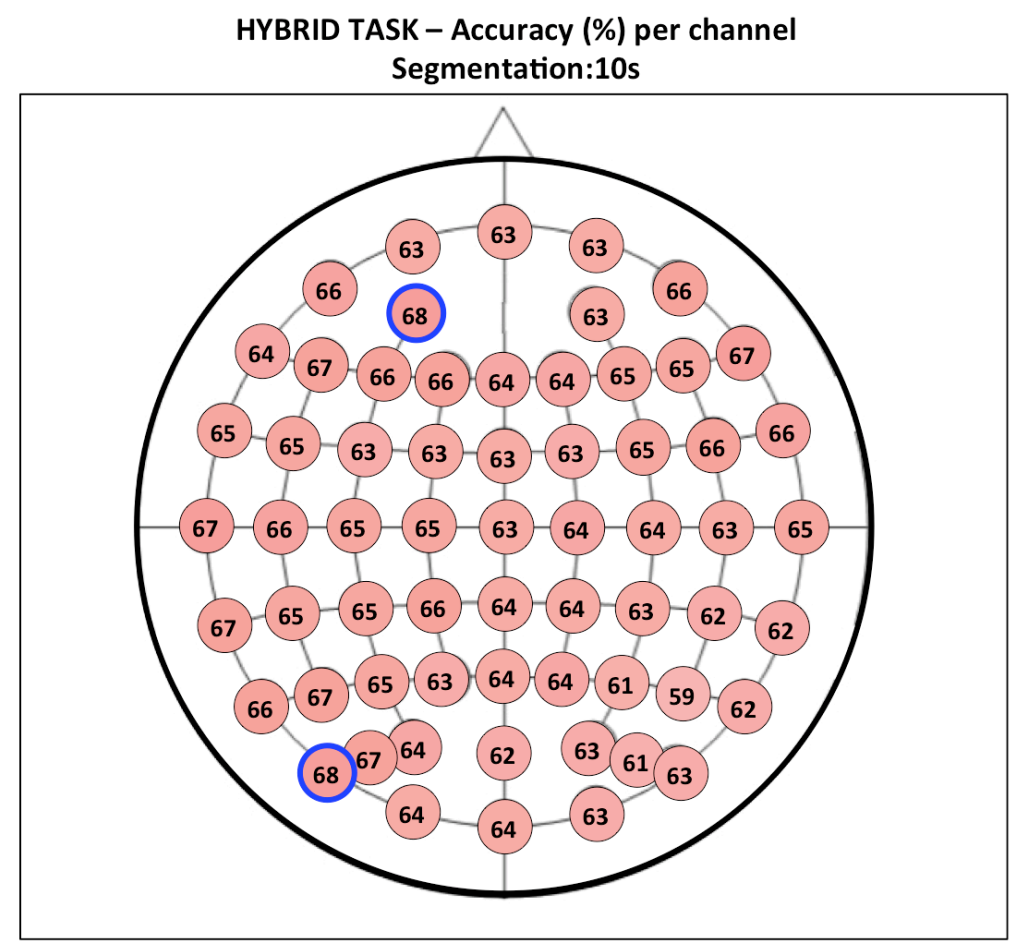

Figure 22 - Hybrid task: Average accuracy (\%) per channel at 10s segmentation - 6 trials (24 choices) per participant, 19 participants. The electrodes with the highest accuracy are highlighted (blue border) and the average accuracy values are displayed in each electrode.

\subsubsection{All tasks comparison}

The following figures present the results achieved by all tasks combined, facilitating comparison. For the P300 task, the results from both feature extraction algorithms were included. The average accuracies based on 10 s segmentation and the best performing channel, per participant, are displayed in Figure 23. 


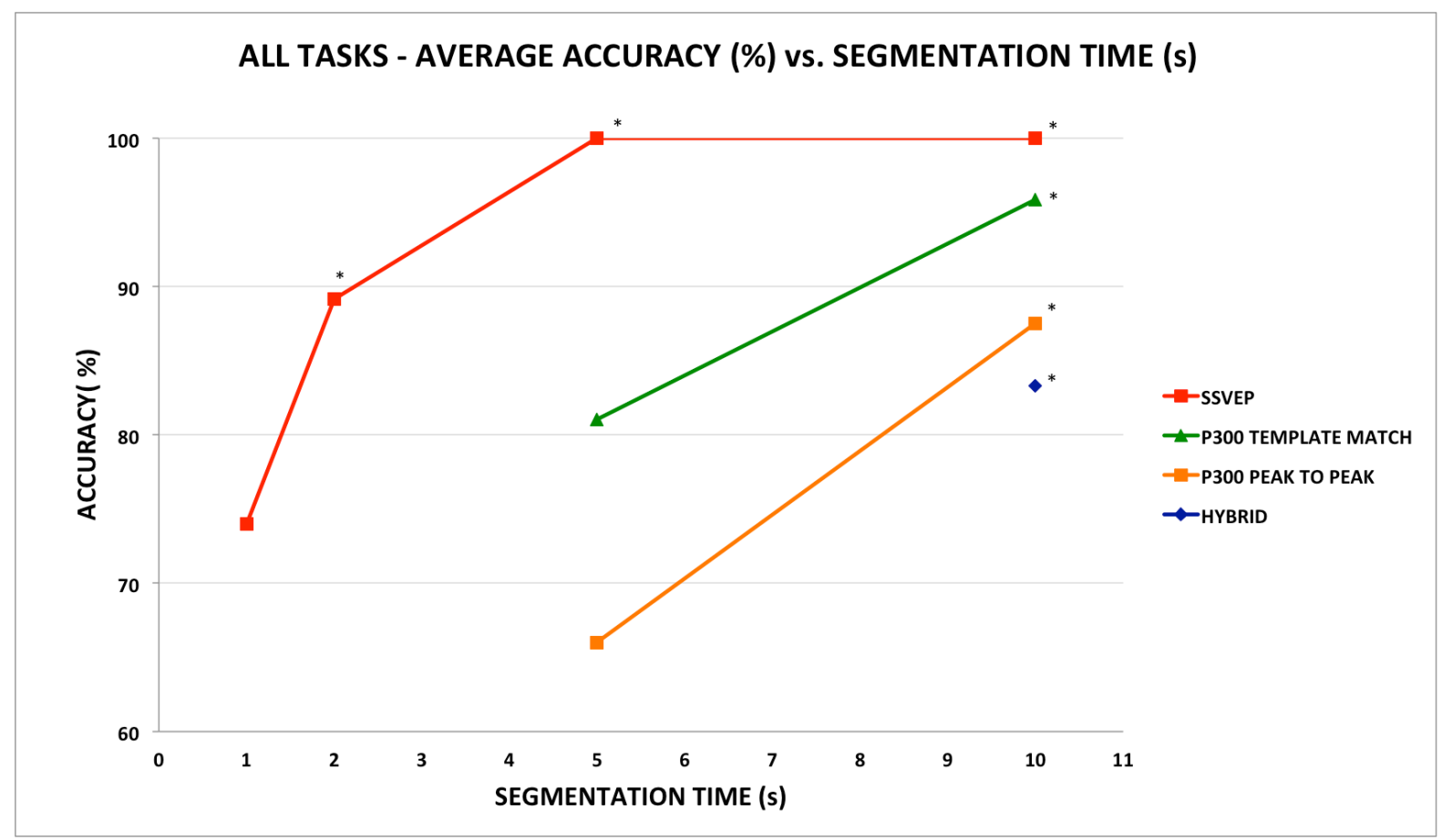

Figure 23 - All tasks: average accuracy (\%) vs. segmentation time (s) curves based on the best performing channel of each participant.

According to the Kruskal-Wallis test, the accuracy was significantly different between the four groups (SSVEP, P300 "template match", P300 "peak to peak" and hybrid) for 10 s segmentation $(H(3)=22,065, p=0,000)$. A second Kruskal-Wallis test, excluding the hybrid task group, did not indicate a significant difference between the three groups, $(H(2)=5,163, p=0,076)$ for accuracy. Further, a third Kruskal-Wallis test between the two SSVEP groups ( $5 \mathrm{~s}$ and $2 \mathrm{~s}$ segmentation) and the two P300 groups (both algorithms for 10 s segmentation) did not indicate a significant difference for accuracy $(H(2)=1,342, p=0,511)$.

Figure 24 details the performance of each participant, per task, for $10 \mathrm{~s}$ segmentation. Seven participants (P06, P07, P08, P11, P13, P14 and P17) achieved the maximum accuracy $(100 \%)$ in both SSVEP and P300 tasks. From this subgroup of participants, that result was achieved regardless of the P300 feature extraction algorithm that was used, except for P07 and P013.

The P300 "peak to peak" algorithm presented a lower performance than the "template match" algorithm on average but not for all participants.

The performance level for the hybrid task was, on average, lower than for other tasks. In addition, it was not the best performing task for any participant, although three participants (P06, P10 and P14) achieved accuracies higher than $90 \%$. 


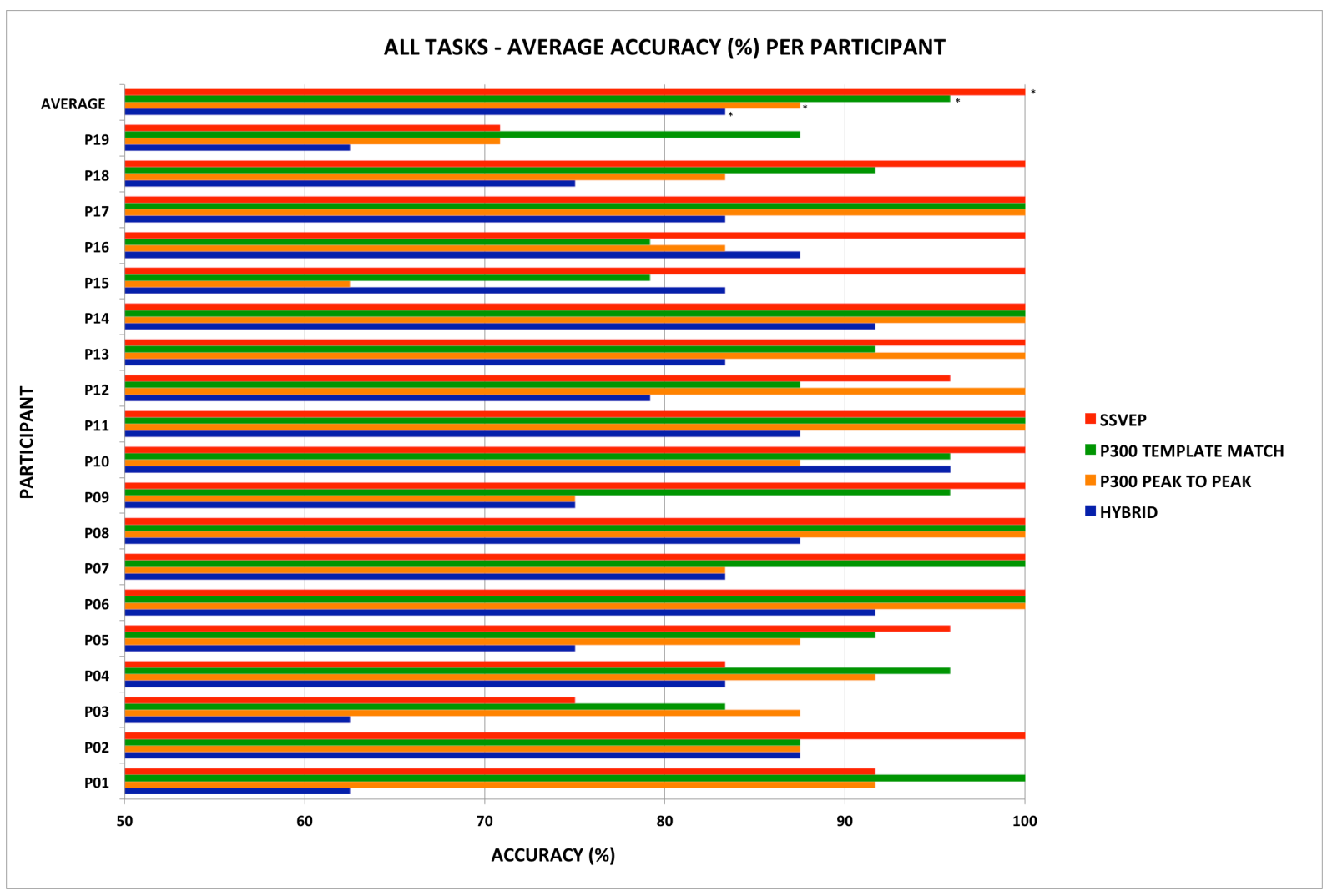

Figure 24 - All tasks: accuracy (\%) achieved by each participant based on their best performing channel and 10 s segmentation.

\subsection{INFORMATION TRANSFER RATE}

Table 1 shows the ITR results for all tasks, based on the highest accuracies achieved per one (or more) participant(s) and on average for all participants (maximum accuracy/ITR and average accuracy/ITR, respectively), using 10s segmentation. Some participants in both the P300 and SSVEP tasks were able to achieve the maximum ITR possible of $12,0 \mathrm{bits} / \mathrm{min}$. 
Table 1 - ITR results from all tasks, based on the highest accuracies achieved (10s segmentation).

\begin{tabular}{l|c|c|c|c}
\hline $\begin{array}{l}\text { TASKS } \\
\text { (Segmentation time: } \\
\text { 10s) }\end{array}$ & $\begin{array}{c}\text { Maximum } \\
\text { Accuracy } \\
(\%)\end{array}$ & $\begin{array}{c}\text { Maximum ITR } \\
\text { (bits/min) }\end{array}$ & $\begin{array}{c}\text { Average } \\
\text { Accuracy (\%) }\end{array}$ & $\begin{array}{c}\text { Average ITR } \\
\text { (bits/min) }\end{array}$ \\
\hline SSVEP & 100 & $\mathbf{1 2 , 0}$ & $100(96-100)$ & $\mathbf{1 2 , 0}(\mathbf{1 0 , 1 - 1 2 , 0 )}$ \\
\hline P300 TEMP. MATCH & 100 & $\mathbf{1 2 , 0}$ & $96(88-100)$ & $\mathbf{1 0 , 1 ( 7 , 6 - 1 2 , 0 )}$ \\
\hline $\begin{array}{l}\text { P300 PEAK TO } \\
\text { PEAK }\end{array}$ & 100 & $\mathbf{1 2 , 0}$ & $88(83-100)$ & $\mathbf{7 , 6 ( 6 , 6 - 1 2 , 0 )}$ \\
\hline HYBRID & 96 & $\mathbf{1 0 , 1}$ & $83(75-88)$ & $\mathbf{6 , 2} \pm \mathbf{2 , 1}$ \\
\hline
\end{tabular}

According to the Kruskal-Wallis test, the ITR was significantly different between the four groups (SSVEP, P300 "template match", P300 "peak to peak" and hybrid) for 10s segmentation $(H(3)=22,065, p=0,000)$. A second Kruskal-Wallis test, excluding the hybrid task group, did not indicate a significant difference between the three groups, $(H(2)=5,163, p=0,076)$ for ITR.

As previously described in sections 6.1.1 and 6.1.2, the SSVEP and P300 tasks were further analysed for shorter segment lengths. The ITR could be estimated based on their respective accuracies, as well. However, it should be remarked that, although the system could theoretically provide a response (output) after each segment, in spite of its length, the stimulation time per location was always kept at 10s. Therefore, this result is presented as ITR ${ }^{* *}$ in Table 2 and in Figure 25. The highest ITR ${ }^{* *}$ of $104,2 \mathrm{bits} / \mathrm{min}$ was achieved by one of the participants in the SSVEP task. Based on P300 task accuracy results (see section 6.1.2), the performance level of the "peak to peak" algorithm was inferior to the "template match" algorithm and, therefore, it was excluded from ITR ${ }^{* *}$ calculation. 
Table 2 - ITR** results from SSVEP and P300 tasks, based on the average accuracies and on the maximum accuracies achieved by one (or more) participant(s), for different segmentation lengths.

\begin{tabular}{|c|c|c|c|c|}
\hline & \multicolumn{3}{|c|}{ SEGMENTATION TIME (s) } \\
\hline & & 5 & 2 & 1 \\
\hline \multirow{4}{*}{$\begin{array}{l}\text { SSVEP } \\
\text { TASK }\end{array}$} & $\begin{array}{c}\text { Maximum } \\
\text { Accuracy (\%) }\end{array}$ & 100 & 100 & 97 \\
\hline & $\begin{array}{c}\text { Maximum } \\
\text { ITR } \\
\text { (bits/min) }\end{array}$ & 24,0 & 60,0 & 104,2 \\
\hline & $\begin{array}{c}\text { Average } \\
\text { Accuracy (\%) }\end{array}$ & $100(83-100)$ & $89(70-95)$ & $74 \pm 18$ \\
\hline & $\begin{array}{l}\text { Average ITR } \\
\text { (bits } / \mathrm{min} \text { ) }\end{array}$ & $\begin{array}{c}24,0(13,0- \\
24,0)\end{array}$ & $36,0 \pm 18,7$ & $52,8 \pm 32,3$ \\
\hline \multirow{4}{*}{$\begin{array}{c}\text { P300 } \\
\text { TASK } \\
\text { (TEMPLAT } \\
\text { E MATCH) }\end{array}$} & $\begin{array}{c}\text { Maximum } \\
\text { Accuracy (\%) }\end{array}$ & 98 & - & - \\
\hline & $\begin{array}{c}\text { Maximum } \\
\text { ITR** } \\
\text { (bits/min) }\end{array}$ & 21,9 & - & - \\
\hline & $\begin{array}{c}\text { Average } \\
\text { Accuracy (\%) }\end{array}$ & $81 \pm 10$ & - & - \\
\hline & $\begin{array}{l}\text { Average ITR }{ }^{* *} \\
\text { (bits/min) }\end{array}$ & $12,5 \pm 4,5$ & - & - \\
\hline
\end{tabular}

. The Mann-Whitney test between two P300 "template match" groups (10s and 5s segmentation) indicated a significant difference for ITR $(U=108, Z=-2,126$, $p=0,034)$. 


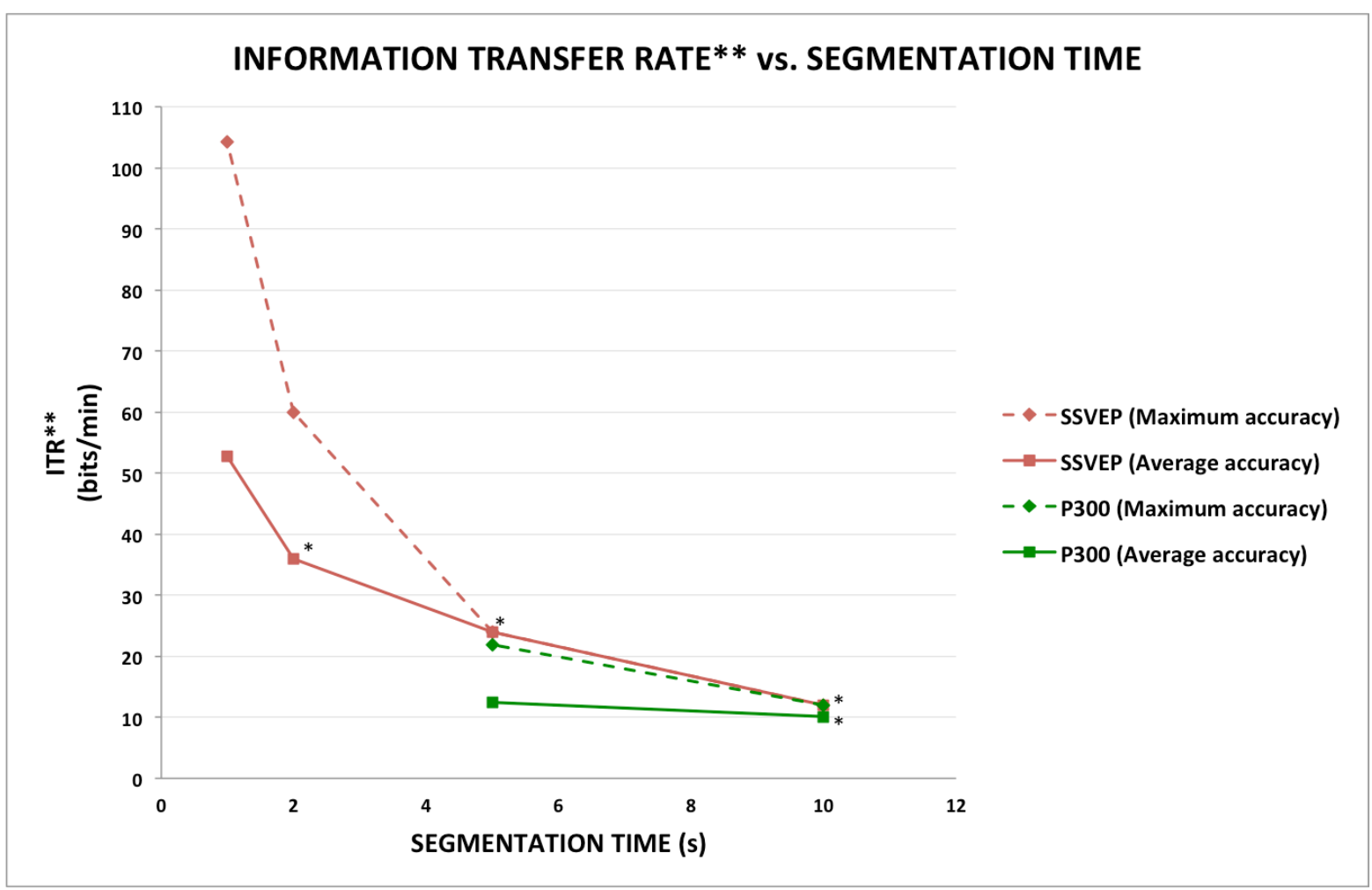

Figure $25-$ ITR $^{\star *}$ vs. segmentation time curves from SSVEP and P300 tasks, based on the average accuracies and on the maximum accuracies achieved by one (or more) participant(s), for different segmentation lengths.

\subsection{NUMBER OF ILLITERATES}

Table 3 shows the number of participants that were not able to achieve at least $70 \%$ of accuracy (I) per task, from a total of 19 participants. None of the participants were classified as illiterates in the SSVEP task if using 10 s segmentation. However, if the segmentation time was reduced, the illiteracy rate increased. Likewise, none of the participants were classified as illiterates in the P300 task if using 10 s segmentation and the "template match" algorithm but, if the segmentation time was reduced, the illiteracy rate also increased. The hybrid task had a lower illiteracy rate performance than other tasks (15,8\%, 10s segmentation).

Additionally, the "peak to peak" algorithm resulted in higher illiteracy rates $(5,3 \%$, 10 s segmentation; $63,2 \%$, 5 s segmentation) if compared to the "template match" algorithm $(0,0 \%$, 10 s segmentation; $15,8 \%$, $5 \mathrm{~s}$ segmentation), although the participants and the data used were the same. 
Further, if the segmentation time was reduced, the illiteracy rate increased, for both P300 and SSVEP tasks. However, while the P300 task achieved an illiteracy rate of $15,8 \%$, if using 5 s segmentation, the SSVEP task illiteracy rate did not raise to this level until 2s segmentation was used. A $X^{2}$ test of illiteracy number according to the task groups including all task groups except for the SSVEP task for 1s segmentation and the P300 task for 5s segmentation using "peak to peak" algorithm, did not indicate a significant difference between groups $\left(X^{2}(6, N=133)=8,127\right.$, $p=0,229)$. The inclusion of any of the two previously excluded groups in the $X^{2}$ test would result in a significant difference between groups for illiteracy.

Table 3 - Number and percentage of illiterates per task from a total of 19 participants.

\begin{tabular}{|c|c|c|c|c|}
\hline & & \\
\hline & & $\begin{array}{c}\text { SEGMENTATION } \\
\text { TIME (s) }\end{array}$ & $\begin{array}{c}\text { I } \\
\text { (Number of } \\
\text { participants) }\end{array}$ & I (\%) \\
\hline \multirow{9}{*}{ 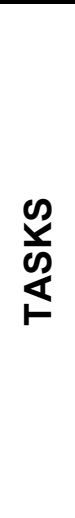 } & \multirow{4}{*}{ SSVEP } & 10 & 0 & 0,0 \\
\hline & & 5 & 1 & 5,3 \\
\hline & & 2 & 3 & 15,8 \\
\hline & & 1 & 7 & 36,8 \\
\hline & \multirow{2}{*}{$\begin{array}{l}\text { P300 (TEMPLATE } \\
\text { MATCH) }\end{array}$} & 10 & 0 & 0,0 \\
\hline & & 5 & 3 & 15,8 \\
\hline & \multirow{2}{*}{ P300 (PEAK TO PEAK) } & 10 & 1 & 5,3 \\
\hline & & 5 & 12 & 63,2 \\
\hline & HYBRID & 10 & 3 & 15,8 \\
\hline
\end{tabular}

\subsection{INDIVIDUAL PREFERENCES}

The participants were asked to complete the individual preferences questionnaire after the experiment, in which they were asked to: a) rank the tasks in order of preference, being RANK 1 the preferred one and RANK 3 the least preferred one and b) answer how they would agree with the statement "I liked to perform the task X", for each task, according to the scale: -2: strongly disagree, -1: slightly disagree, 0: neutral, 1: slightly agree and 2: strongly agree. The final results are presented in Table 4. 
Table 4 - Results of the individual preference assessment based on questionnaires completed after the experiment. After the experiment, the participants were asked how they agreed with the statement "I liked to perform the task X", for each task, according to the likert scale: -2 : strongly disagree, -1 : slightly disagree, 0 : neutral agreement, 1 : slightly agree and 2 : strongly agree.

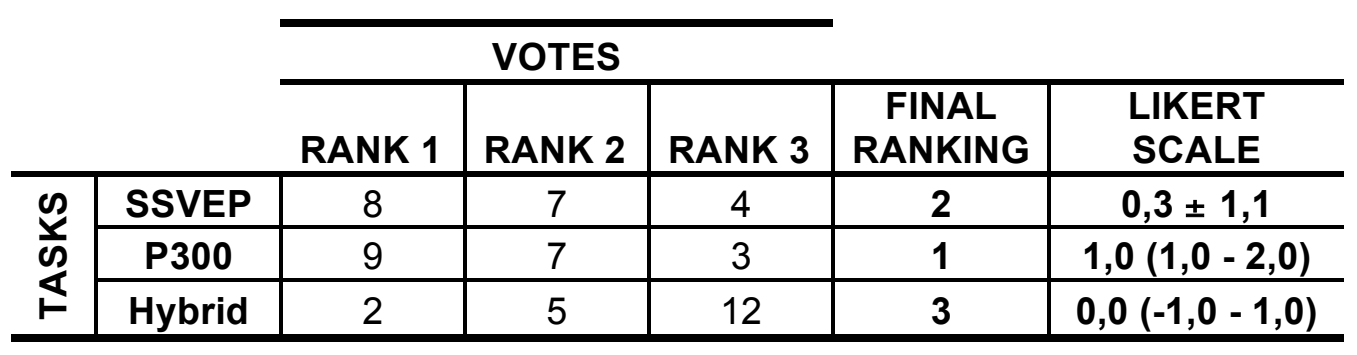

According to a $\mathrm{X}^{2}$ test, the individual preferences differed significantly between the three tasks $\left(X^{2}(2, N=57)=6,333, p=0,042\right)$, but did not reach significance between the SSVEP and hybrid tasks $\left(X^{2}(2, N=38)=3,257, p=0,071\right)$.

\subsection{BEST CHANNELS}

Table 5 shows the full ranking of all 62 channels per task, according to their respective accuracies. Note: M1 and M2 electrodes were used for re-referencing and were excluded from the performance analysis.

Table 5 - Full ranking of all 62 channels per task, according to their respective average accuracies.

\begin{tabular}{c|c|c|c|c|c|c|c|c}
\hline & \multicolumn{2}{|c|}{ SSVEP } & \multicolumn{2}{c|}{$\begin{array}{c}\text { P300 TEMP. } \\
\text { MATCH }\end{array}$} & \multicolumn{2}{c|}{$\begin{array}{c}\text { P300 PEAK TO } \\
\text { PEAK }\end{array}$} & \multicolumn{2}{c}{ HYBRID } \\
\hline RANK & Channel & $\begin{array}{c}\text { Accuracy } \\
(\%)\end{array}$ & Channel & $\begin{array}{c}\text { Accuracy } \\
(\%)\end{array}$ & Channel & $\begin{array}{c}\text { Accuracy } \\
(\%)\end{array}$ & Channel & $\begin{array}{c}\text { Accuracy } \\
(\%)\end{array}$ \\
\hline $\mathbf{1}$ & Oz $^{*}$ & 100 & Po8 & 79 & Po8 & 83 & Af3 & 68 \\
\hline $\mathbf{2}$ & O1 $^{*}$ & 96 & Cpz & 77 & O1 & 72 & Po7 & 68 \\
\hline $\mathbf{3}$ & O2* $^{*}$ & 96 & Ft8 & 77 & P8 & 72 & F8 & 67 \\
\hline $\mathbf{4}$ & Po8 $^{*}$ & 92 & Fc5 & 77 & Po7 & 71 & T7 & 67 \\
\hline $\mathbf{5}$ & Poz $^{*}$ & 83 & Ft7 & 77 & Oz & 70 & P5 & 67 \\
\hline $\mathbf{6}$ & Po6 $^{*}$ & 83 & C2 & 77 & Po5 & 70 & F5 & 67 \\
\hline $\mathbf{7}$ & Po7 $^{*}$ & 83 & Fcz & 77 & Po6 & 70 & Po5 & 67 \\
\hline $\mathbf{8}$ & Po4 $^{*}$ & 79 & Fc1 & 77 & O2 & 69 & Tp7 & 67 \\
\hline $\mathbf{9}$ & P8 & 74 & F8 & 76 & Po3 & 69 & F3 & 66 \\
\hline $\mathbf{1 0}$ & P6 & 71 & Cz & 76 & Poz & 64 & Fc6 & 66 \\
\hline $\mathbf{1 1}$ & Po3 & 71 & Af4 & 76 & Po4 & 63 & Cp1 & 66 \\
\hline $\mathbf{1 2}$ & Po5 & 71 & F4 & 76 & P6 & 63 & Af8 & 66 \\
\hline
\end{tabular}




\begin{tabular}{|c|c|c|c|c|c|c|c|c|}
\hline 13 & P4 & 66 & Fc6 & 76 & P1 & 62 & P7 & 66 \\
\hline 14 & FCz & 63 & F6 & 76 & $C_{p z}$ & 62 & F1 & 66 \\
\hline 15 & F1 & 63 & C1 & 75 & P2 & 61 & Ft8 & 66 \\
\hline 16 & Af3 & 63 & Cp2 & 75 & Cp1 & 61 & Af7 & 66 \\
\hline 17 & Fc2 & 62 & F2 & 75 & C2 & 60 & C5 & 66 \\
\hline 18 & Fz & 62 & Fc2 & 75 & P5 & 60 & F4 & 65 \\
\hline 19 & Fc1 & 61 & Fc3 & 75 & P3 & 60 & T8 & 65 \\
\hline 20 & $\mathrm{Cz}$ & 61 & Cp1 & 74 & $\mathbf{P z}$ & 60 & C3 & 65 \\
\hline 21 & F4 & 61 & Fc4 & 74 & $\mathrm{Cz}$ & 59 & F6 & 65 \\
\hline 22 & F3 & 60 & F1 & 74 & Cp2 & 59 & Fc4 & 65 \\
\hline 23 & F2 & 59 & C4 & 74 & Af4 & 59 & C1 & 65 \\
\hline 24 & Fc3 & 59 & P8 & 74 & P4 & 58 & Cp3 & 65 \\
\hline 25 & P7 & 59 & 01 & 74 & Fcz & 58 & P3 & 65 \\
\hline 26 & Af4 & 59 & $\mathrm{Oz}$ & 74 & Fc2 & 58 & Ft7 & 65 \\
\hline 27 & F5 & 59 & F3 & 74 & Fc4 & 58 & Cp5 & 65 \\
\hline 28 & P2 & 58 & T7 & 73 & C4 & 58 & Fc5 & 65 \\
\hline 29 & C2 & 58 & 02 & 73 & F6 & 58 & $\mathrm{~F} 2$ & 64 \\
\hline 30 & P5 & 57 & $\mathbf{P z}$ & 73 & P7 & 57 & P2 & 64 \\
\hline 31 & Fc5 & 56 & C5 & 73 & C1 & 57 & Fz & 64 \\
\hline 32 & Fc4 & 56 & $\mathbf{F z}$ & 73 & Cp3 & 57 & F7 & 64 \\
\hline 33 & P3 & 56 & C3 & 73 & Fc6 & 57 & $\mathrm{C} 2$ & 64 \\
\hline 34 & F6 & 55 & F5 & 73 & C6 & 57 & Po3 & 64 \\
\hline 35 & Fc6 & 55 & Po7 & 73 & Fp2 & 56 & $\mathrm{Oz}$ & 64 \\
\hline 36 & $\mathrm{~F} 7^{*}$ & 54 & Af3 & 73 & F2 & 56 & $\mathbf{P z}$ & 64 \\
\hline 37 & P1 & 54 & P1 & 72 & Af3 & 56 & 01 & 64 \\
\hline 38 & Af8 & 54 & Cp3 & 72 & Ft8 & 56 & Cp2 & 64 \\
\hline 39 & Fp2 & 54 & Fpz & 72 & Fz & 56 & C4 & 64 \\
\hline 40 & Cp2 & 54 & Po3 & 72 & Fc1 & 56 & Cpz & 64 \\
\hline 41 & F8 & 53 & Fp2 & 72 & F4 & 55 & $\mathrm{Cz}$ & 63 \\
\hline 42 & Af7 & 52 & Af8 & 72 & F3 & 55 & Af4 & 63 \\
\hline 43 & $\mathbf{P z}$ & 52 & Cp4 & 71 & Fpz & 55 & $F p z$ & 63 \\
\hline 44 & Ft8 & 52 & $\mathrm{C} 6$ & 71 & Af8 & 55 & Fc1 & 63 \\
\hline 45 & C3 & 52 & P2 & 71 & C3 & 55 & C6 & 63 \\
\hline 46 & Ft7 & 50 & T8 & 71 & Af7 & 54 & Fcz & 63 \\
\hline 47 & Cp4 & 50 & Po5 & 71 & $F 1$ & 54 & Cp4 & 63 \\
\hline 48 & C1 & 50 & Po6 & 71 & F5 & 54 & Fp1 & 63 \\
\hline 49 & $\mathrm{Cpz}$ & 50 & F7 & 70 & Fc3 & 54 & Fp2 & 63 \\
\hline 50 & Cp6 & 50 & Cp5 & 70 & Cp5 & 53 & Fc2 & 63 \\
\hline 51 & Fp1 & 50 & Poz & 70 & F8 & 53 & P1 & 63 \\
\hline 52 & C5 & 48 & P3 & 70 & Cp4 & 53 & 02 & 63 \\
\hline 53 & Tp8 & 46 & Af7 & 69 & C5 & 52 & Po4 & 63 \\
\hline 54 & C4 & 45 & Po4 & 68 & Ft7 & 52 & Fc3 & 63 \\
\hline 55 & Cp3 & 45 & P6 & 68 & F7 & 51 & Po8 & 63 \\
\hline
\end{tabular}




\begin{tabular}{c|c|c|c|c|c|c|c|c}
$\mathbf{5 6}$ & T8 & 43 & P4 & 67 & Cp6 & 51 & Poz & 62 \\
\hline $\mathbf{5 7}$ & T7 & 43 & P5 & 66 & Fc5 & 50 & Cp6 & 62 \\
\hline $\mathbf{5 8}$ & Cp1 & 42 & Cp6 & 65 & T8 & 50 & P8 & 62 \\
\hline $\mathbf{5 9}$ & Fpz & 38 & Fp1 & 64 & Fp1 & 49 & Tp8 & 62 \\
\hline $\mathbf{6 0}$ & Cp5 & 38 & P7 & 64 & Tp8 & 49 & P4 & 61 \\
\hline $\mathbf{6 1}$ & Tp7 & 38 & Tp7 & 63 & T7 & 45 & Po6 & 61 \\
\hline $\mathbf{6 2}$ & C6 & 33 & Tp8 & 61 & Tp7 & 43 & P6 & 59 \\
\hline
\end{tabular}

From the ranking above, the best sets of 8,16 and 32 channels can be selected, according to the task. A visual representation of these selections is shown in Figure 26. Further, the average accuracy was calculated (Table 6) considering the best performing electrode from reduced sets of the best performing 32, 16 and 8 electrodes, according to the ranking presented in Table 5. 


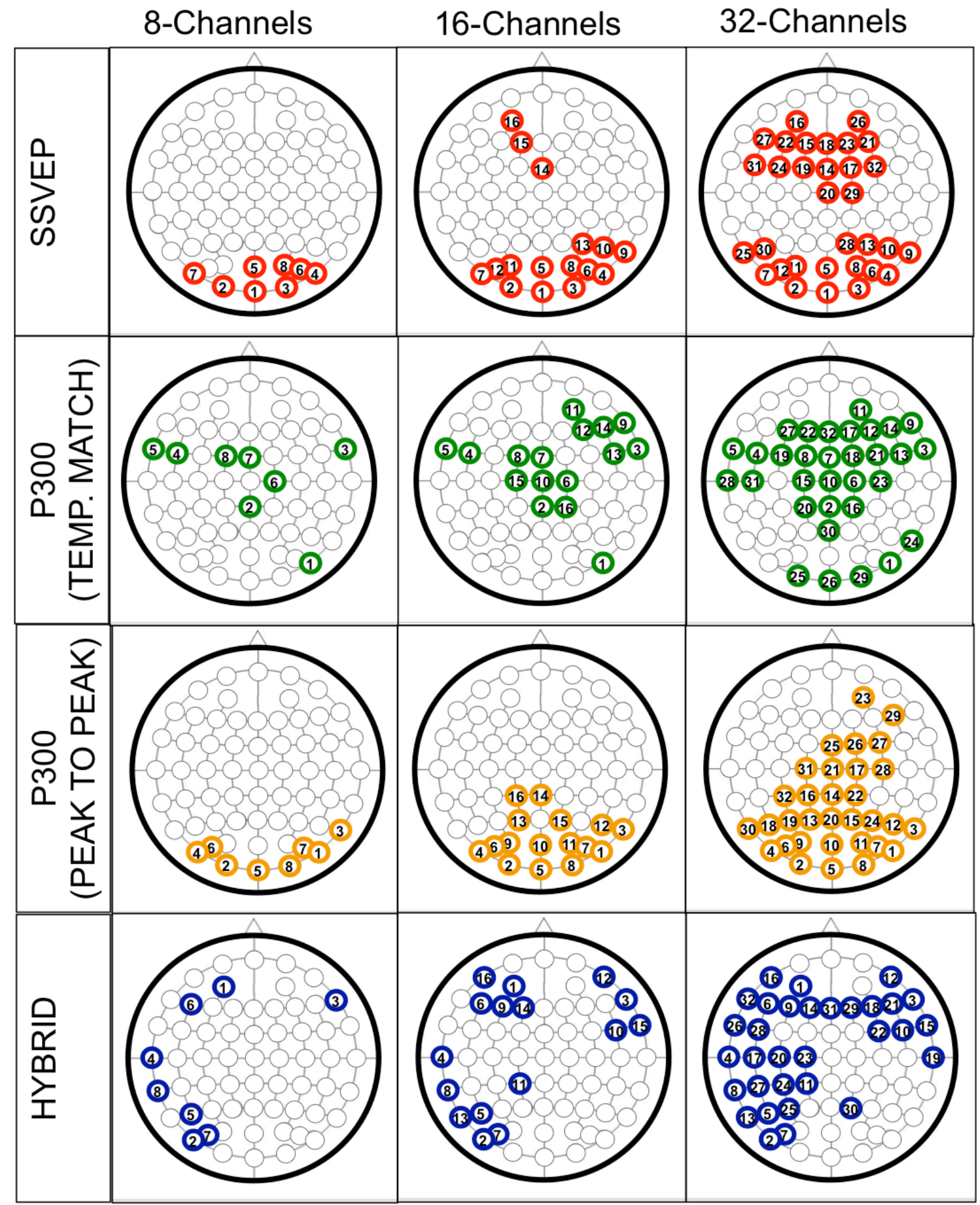

Figure 26 - Visual representation of best performing sets of 8,16 and 32 channels per task, according to their average accuracies. The ranking positions are displayed inside their respective electrodes. 
Table 6 - Average accuracy considering the best performing electrode per participant from the full set of 62 electrodes and reduced sets of the best performing 32,16 and 8 electrodes, according to the ranking presented in Table 5.

\begin{tabular}{l|c|c|c|c}
\hline \multirow{2}{*}{ TASK } & \multicolumn{4}{c}{ Average accuracy (\%) per set of electrodes } \\
\cline { 2 - 5 } & 62-channel & 32-channel & 16-channel & 8-channel \\
\hline SSVEP & $100(96-100)$ & $100(96-100)$ & $100(96-100)$ & $100(96-100)$ \\
\hline $\begin{array}{l}\text { P300 } \\
\text { (TEMPLATE MATCH) }\end{array}$ & $96(88-100)$ & $92(88-100)$ & $92(83-100)$ & $89 \pm 9$ \\
\hline $\begin{array}{l}\text { P300 } \\
\text { (PEAK TO PEAK) }\end{array}$ & $88(83-100)$ & $88(83-100)$ & $88(83-100)$ & $88(75-100)$ \\
\hline HYBRID & $83(75-88)$ & $83(75-88)$ & $80 \pm 11$ & $77 \pm 11$ \\
\hline
\end{tabular}

A series of four Kruskal-Wallis tests was performed to verify whether the difference between the electrode sets for accuracy reaches significance, per task. None of the tests indicated significant differences for accuracy if the sets of electrodes were reduced. The statistical tests results are presented below:

a) SSVEP task: $(H(3)=0,000, p=1,000)$;

b) P300 task (template match): $(H(3)=2,256, p=0,521)$;

c) P300 task (peak to peak): $(H(3)=0,293, p=0,961)$;

d) Hybrid task: $(\mathrm{H}(3)=2,664, \mathrm{p}=0,446)$. 


\section{DISCUSSION}

The main goals of this study were: a) to select the best brain stimulation method for eventual development of a discrete brain-machine interface that does not require neurofeedback training for the control of two binary parameters (four choices) and b) to select the best subsets of 8,16 and 32 channels out of 62 channels in terms of accuracy for portability increase and setup reduction purposes. We systematically compared three different tasks (SSVEP, P300 and Hybrid) that were designed with the same number of choices, stimulation time per choice and number of trials, based on four performance parameters (accuracy, ITR, "illiteracy" rate and individual preferences), within one group of participants $(n=19)$. Additionally, two different algorithms, namely the "peak to peak" and the "template match" were used for the P300 feature extraction. Finally, the accuracy as a function of the number of channels was evaluated and the best subsets of 32, 16 and 8 channels out of 62 were selected.

Our results are discussed below.

\subsection{ALL TASKS COMPARISON}

The performance of both SSVEP and P300 tasks did not significantly differ for 10 s segments, if the "template match" algorithm was used for the feature extraction of the latter. However, this situation changed in favour of the SSVEP task if segment length was reduced to $5 \mathrm{~s}$. Actually, for the SSVEP task the maximum accuracy level $(100 \%)$ was maintained for 5 s segmentation and it was still above the $70 \%$ "illiteracy threshold", on average, for the shortest segment length evaluated (1s). Further, the accuracy decrease with the segment length reduction did not prevent the ITR $^{* *}$ of the SSVEP task from increasing up to a maximum of 52,8 bits/min (1s segmentation). Altogether, it made the SSVEP task the best performer according to three out of four criteria (accuracy, ITR and illiteracy) and compared to the P300 task it only scored worse on the individual preferences assessment. The group of participants $(n=19)$ presented a neutral agreement to the statement: I liked to perform the SSVEP task". 
Although this was not an issue during the short trials of this study, this information is relevant for the use of a $\mathrm{BMI}$ on the longer term. The user fatigue or unwillingness to use the interface over time could disrupt its performance and make it impractical.

On the other hand, the same group of participants slightly agreed that they liked to perform the P300 task. However, while the performance of the SSVEP and P300 tasks was similar for 10 s segmentation, the P300 performance strongly decreased for 5 s segmentation and the ITR $^{* *}$ did not significantly improve, reaching 12,5 bits/min only. This is not entirely surprising: the P300 detection requires segment averaging to achieve signal-to-noise reduction. In five seconds, according to the ISI used $(14 / 75 \mathrm{~ms})$ and the number of symbols in the P300 matrix (6, including the dummies), less than 5 segments including the ERP are expected to be present. One could argue that the P300 task performance could be increased with ISI reduction. However, this could lead to decreased ERP amplitude and increased overlap between two consecutive ERP waveforms. In this study, the interval between two consecutive flashes of the same symbol was maximized using a pseudorandomization algorithm. It minimized the chance of overlap between two consecutive P300 waveforms, but this possibility was not excluded. Thus, the smaller the segment length, the stronger the effects of a possible ERP overlap in the averaged P300 waveform and the possibilities to avoid it are limited by the number of choices and ISI.

The hybrid task demonstrated the worst performance according to all criteria. The hypothesis that the responses to each stimulus would be located at different sites on the scalp and that a hybrid approach would result in superior performance was not demonstrated. The best performing electrodes were mainly peripheral to the best performing electrodes from the single-stimulus tasks and did not achieve superior results. It can be inferred from the results that: a) the ERPs from the SSVEP task were stronger in the occipital area but were also present in other sites, distorting the P300 ERP waveforms; b) the dual task increased the cognitive workload, decreasing the P300 amplitudes and changing the ERP scalp distribution and c) the dual task distracted the visual focus from the virtual buttons, decreasing the SSVEPs. Additionally, the participants did not like to perform the task, discouraging further investigation of tasks based on simultaneous attention to P300 and SSVEP stimuli. 


\subsection{COMPARISON OF DIFFERENT P300 ALGORITHMS}

The results show that different feature extraction algorithms varied in their effectiveness when applied to data of different participants and to the same data of one participant, as well. The reasons behind this variation in effectiveness are related to the interaction between the characteristics of the P300 waveform and the specific features extracted by each algorithm. The "peak to peak" algorithm considers only two points (minimum amplitude and maximum amplitude) per segment window, being effective in individuals that present a high amplitude variation, regardless of the latency variability. In the "template match" algorithm, all points of the segment are taken into account and compared (weighted) to their respective points in the individual template from the participant. Thus, it is likely that, for the template match algorithm to be the most effective, participants need to have a distinctive waveform and low latency variability across trials. Furthermore, the different algorithms demonstrated better results on electrodes located in different areas on the scalp. The best performing electrodes for the "peak to peak" variant were clearly located over posterior sites, mainly in the parieto-occipital region, while the "template match" algorithm performed better on electrodes over the frontal and central areas. Interestingly, these findings are supported by literature. Firstly, P300 amplitude typically increases in magnitude from the frontal to the parietal sites (Johnson 1993) and, therefore, it is expected that the "peak to peak" algorithm presents better results in posterior sites. Similarly, P300 latency changes across the scalp and is shorter over frontal areas but longer over parietal areas (Mertens and Polich 1997). These empirical findings are supported by the theoretical cognitive model of P300 that divides the canonical ERP in two subcomponents, namely P3a and P3b, which are generated in distinct neural loci (Polich 2007). According to the proposed model, the earlier P3a originates from stimulus-driven frontal attention mechanisms during task processing. The attention-driven stimulus signal is then transmitted to temporal and parietal structures related to P3b, which is associated with memory operations. The $\mathrm{P} 3 \mathrm{a}$ has a central maximum and peaks earlier, whereas $\mathrm{P} 3 \mathrm{~b}$ has a parietal maximum and peaks later. The fact that the "template match" algorithm performed better in frontocentral electrodes indicates that these sites had a more distinctive ERP waveform. Given the theoretical cognitive P300 model, the reduced overlap in the 
earlier and shorter $\mathrm{P} 3 \mathrm{a}$ subcomponent that is more pronounced in those sites concomitantly with a lower influence of the later and longer P3b subcomponent may justify the "template match" algorithm performance over different electrodes on the scalp.

In this study, the "template match" algorithm demonstrated superior and less variable results. The template could be generated from the task data because it was an offline study and 4 minutes of data (6 trials, 40 s per trial) were used for this purpose. The fact that online operation would require a short training trial to build the individual ERP template for each user is compensated by the positive impact on performance.

\subsection{DOES "BCI ILLITERACY" EXIST?}

Several studies report that approximately $20 \%$ of the users do not achieve at least $70 \%$ of accuracy and are, therefore, unable to attain control of the $\mathrm{BCl}$ (Allison, Luth, Valbuena, Teymourian, Volosyak and Graser 2010; Vidaurre and Blankertz 2010). There is considerable debate about how to name this phenomenon and some of the terms used by different groups are "BCl illiteracy" and "BCl inefficiency" (Kaufmann et al. 2013), but there is general agreement that this is an issue of concern. In this study, absolutely no participant was below the illiteracy/inefficiency threshold neither in the SSVEP nor in the P300 task if 10 s of stimulation and segmentation were used. Even if the hybrid task is considered, which attained the lowest average accuracy performance, less than $20 \%$ of the participants failed to achieve $70 \%$ of classification accuracy. Clearly, the segmentation time and the feature extraction algorithm used presented a strong influence on the final result. The variability was evident between participants across different tasks and exceptions might exist, but our results indicate that the participants are able to attain control of the $\mathrm{BCl}$ regardless of the stimulus used if enough stimulation time is allowed, the proper electrode is analysed and a feature extraction algorithm according to the individual response is used. Our findings are in agreement with the recent results from (Gembler et al. 2015), where a SSVEP-BCI automatically determines individual 
user parameters and all users attained control over the system, reaching accuracies above $85 \%$.

\subsection{NUMBER OF ELECTRODES}

The best channels analysis demonstrated that the use of 62 channels provides excessively redundant information and the accuracy results for reduced sets as small as 8 electrodes did not significantly differ from the full 62-channel set for any of the tasks. The use of small sets of electrodes reduces the setup time, improves the quality of the user experience and facilitates the data processing as well, which are all valuable for online operation.

\subsection{FINAL CONSIDERATIONS AND POSSIBLE FUTURE DEVELOPMENTS}

This study considered the offline operation of a BMI with four possible choices. Further works could implement online operation and expand the number of choices. In this case, the relative superiority of the SSVEP-based task would be limited by the refresh rate, while the $\mathrm{P} 300$-based task could benefit from more information (bits) being conveyed through bigger matrices. However, the new emerging LCD technologies with refresh rates as fast as $240 \mathrm{~Hz}$ can bring the SSVEP-based BMIs to a new ITR level and should be explored.

Additionally, while the hybrid task did not demonstrate any advantage according to the presented design, other hybrid possibilities exist and this study did not intend to exhaust them. The P300 task demonstrated higher variability across participants for the best performing electrode, but achieved similar results to the SSVEP task for longer segment lengths. These results suggest that SSVEP should be used for commands that require fast detection, while P300 could be used for other less restrictive commands. Therefore, the development of online BMls based on both stimuli for separate and not simultaneous commands is encouraged. 
Additionally, the data pre-processing consisted of downsampling, re-referencing and simple filtering without further use artifacts or data segments rejection. Besides, the feature extraction algorithms used require low computational load. Thus, further implementation in real-time operating systems should be feasible without compromising performance. In case of embedded applications, it should be remarked that the "template match" algorithm, differently from the "peak-to-peak" and the SSVEP feature extraction algorithms, requires a calibration step for the individual user template generation.

Further, error correction algorithms, machine learning techniques and feedback could be applied to improve performance and/or the user experience.

\subsection{STRENGTHS AND LIMITATIONS OF THIS STUDY}

The results were based on data acquired from the same experimental setup and each participant performed all tasks, allowing a systematic comparison between them.

Finally, the participants in this experiment were healthy and mainly young adults. Therefore, the results might not be reflective of neurologically impaired or elder populations, which would typically include the target users of BMls, but provided relevant information about the functioning healthy brain. 


\section{CONCLUSION}

The present study systematically compared three different tasks (SSVEP, P300 and hybrid) for the control of a four-choice EEG-based BMI on the same performance parameters (accuracy, ITR, illiteracy/inefficiency and individual preferences) within one group of 19 participants.

The SSVEP task outperformed the other tasks in accuracy, ITR and illiteracy/inefficiency. All participants attained control of the system with both SSVEP and P300 tasks. The hybrid task did not demonstrate any advantage according to the presented design, but the development of online BMls based on both P300 and SSVEP stimuli for separate (and not simultaneous) commands is encouraged.

The accuracy of all tasks did not significantly deteriorate if a reduced set with the 8 best performing electrodes out of 62 was used, which is relevant for the equipment setup time reduction, user experience improvement and data processing facilitation.

All the results were obtained from offline analysis of EEG data and are relevant for further development of online BMls. The data processing and the feature algorithms proposed are suitable for real-time operation without compromising the performance of the system. 


\section{APPENDIX A. BRAIN RHYTHMS}

The brain waves or brain rhythms arise from the synchronization of the oscillatory activity of large populations of neurons. There are five major brain waves distinguished by their different frequency ranges and spatial distributions and are often correlated with different functional states of the brain (Buzsalki 2006; Rao 2013) :

- Alpha ( $\alpha$ ) waves: 8-13 Hz. The alpha rhythm appears in the posterior half of the head and is typically recorded from the occipital region in awaken persons when they are relaxed and with their eyes closed. It commonly appears as a round or sinusoidal shaped signal. A particular kind of alpha wave that is popular in BMI applications is known as the mu $(\mu)$ rhythm $(8-12 \mathrm{~Hz})$. The rolandic (central) mu rhythm is found over sensory motor areas around the central sulcus in the absence of movement and is decreased or abolished when the subject performs a movement or imagines performing a movement.

- Beta $(\beta)$ waves: $13-30 \mathrm{~Hz}$. The beta rhythm is detectable over the frontal, central and parietal regions and is the usual waking rhythm of the brain that is associated with active thinking or concentrating, problem solving and alertness. Importantly, a central beta rhythm is related to the mu rhythm and can be blocked by motor activity or tactile stimulation.

- Theta $(\theta)$ waves: 4-8 $\mathrm{Hz}$. The theta waves have been associated with drowsiness or "idling".

- Delta $(\delta)$ waves: $0,5-4 \mathrm{~Hz}$. They are primarily associated with deep sleep in adults but may be present in the waking state. Delta waves occur synchronously over the entire neocortex.

- Gamma (y) waves: $30-100 \mathrm{~Hz}$ or higher. The gamma waves have been reported in tasks involving short-term memory and multisensory integration. High gamma activity $(70 \mathrm{~Hz}$ and above) has also been reported for motor tasks. It has low amplitude and is the most characteristic field pattern of the waking, activated neocortex. 


\section{APPENDIX B. ELECTROENCEPHALOGRAPHY (EEG)}

The recording of electrical activity from the exposed cerebral cortex of animals was first reported in 1875, by the English physiologist Richard Caton. The human EEG was first described by Austrian psychiatrist Hans Berger in 1929 (Bear et al. 2016; Purves 2004).

The Electroencephalography (EEG) is a popular non-invasive technique for brain signals acquisition through electrodes placed on the scalp. The EEG signals reflect the summation of post-synaptic potentials of thousands neurons that are oriented radially to the scalp. Currents tangential to the scalp are not detected by EEG and currents originated deep in the brain are also not detected because the electric fields intensity decays with the square of the distance from the source. Thus, the EEG primarily captures the electrical activity in the cortex, whose columnar arrangement of neurons and proximity to the skull favour recording by EEG. The major advantage of electroencephalography is its great simplicity. Its most serious limitation is its poor spatial resolution (in the square centimeter range) but the temporal resolution is good (in the milliseconds range) (Purves 2004; Rao 2013).

The poor spatial resolution of the EEG is caused primarily by the different layers of tissue (meninges, cerebrospinal fluid, skull, scalp) between the signal sources and the sensor placed on the scalp - see Figure 27. It takes many thousands of underlying neurons, activated together, to generate an EEG signal that is big enough to be detected and, additionally, the EEG signal amplitude strongly depends, in part, on how synchronous the activity of those underlying neurons is (Bear, Connors and Paradiso 2016). 


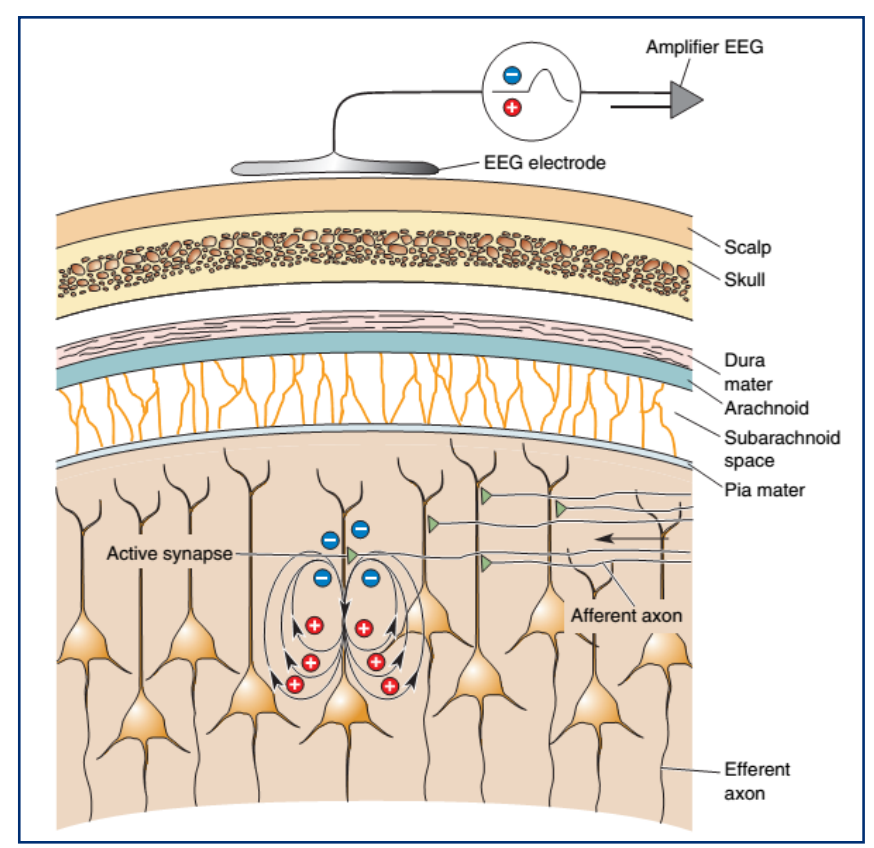

Figure 27 - Layers of non-neural tissues between active synapses and the EEG electrode (Bear, Connors and Paradiso 2016)

The international $10-20$ system is the conventional electrode setting recommended by the International Federation of Electroencephalography and Clinical Neurophysiology for 21 electrodes placement (see Figure 28). The letters in each location correspond to specific brain regions, according to the following classification: "A" represents the mastoids, "C" the central region, "Pg" the nasopharyngeal, "P" the parietal, "F" the frontal, "Fp" the frontal polar and "O" the occipital area. For setting a larger number of the electrodes, the additional electrodes are placed equidistantly between the conventional ones. The Figure 28 also presents a larger setting for 75 electrodes including the reference electrodes based on the guidelines by the American EEG society (Nicolas-Alonso and Gomez-Gil 2012; Sanei and Chambers 2007). 


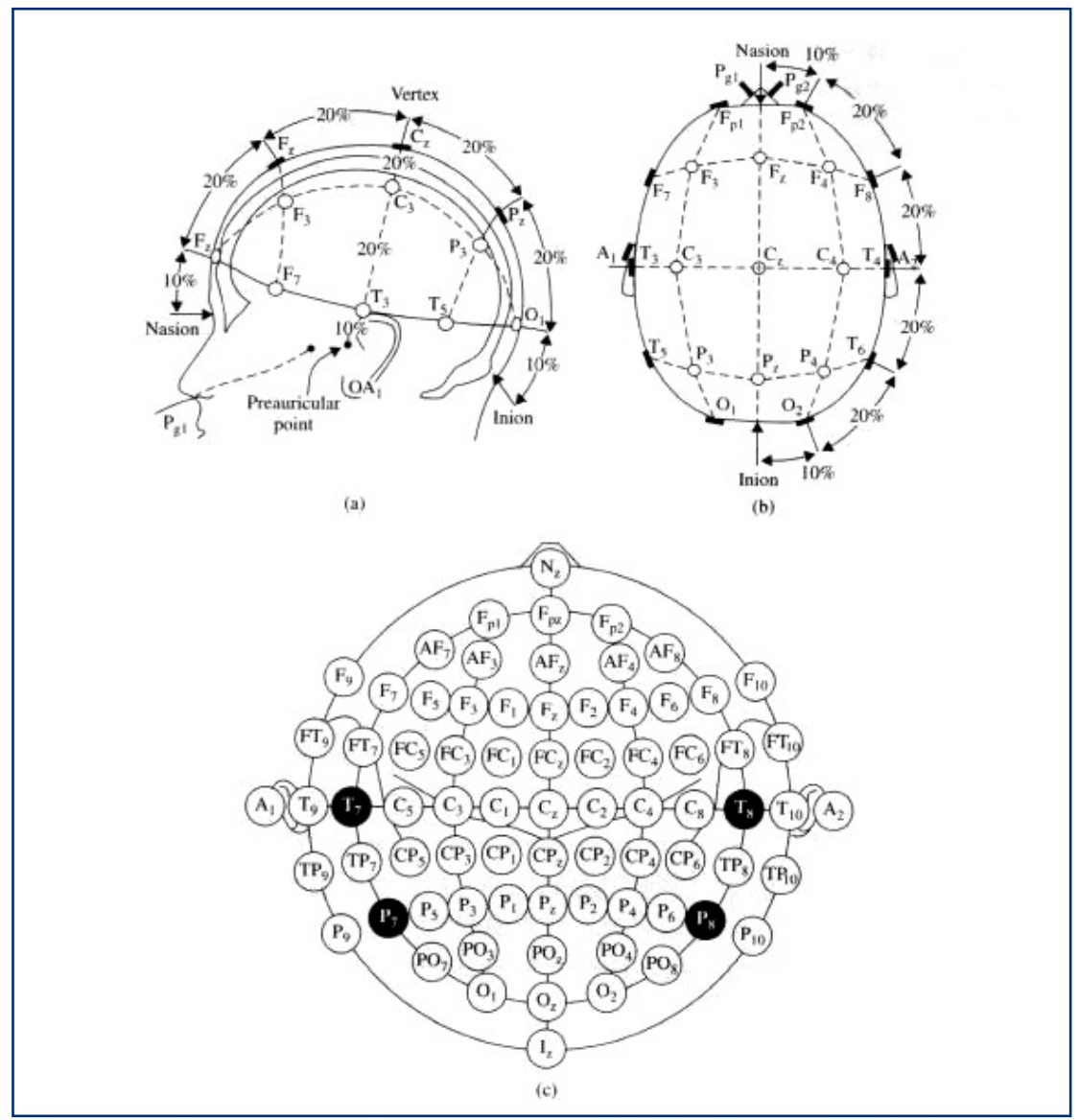

Figure 28 - Electrodes positioning over the scalp based on the international 10-20 system (Sanei and Chambers 2007) 


\section{APPENDIX C. NEAR INFRARED SPECTROSCOPY (NIRS)}

Near infrared spectroscopy is an optical technique used for the measurement of variations in the blood oxygenation level caused by neural activity increase.

FNIRS is able to capture the hemodynamic changes via the coupling of infrared light emission and detection. It is based on the light absorption rate difference between oxygenated and deoxygenated hemoglobin ( $\mathrm{HbO}$ and $\mathrm{HbR}$ ) and the transparency of biological tissue in the 700-1000 nm range (near-infrared). Infrared light can penetrate the human skull to a depth of approximately $1-3 \mathrm{~cm}$ below the skin surface and, therefore, it can reach the outer cortical layer. The infrared light is absorbed differently according to the oxygen concentration in the blood, providing a measure of the subjacent neural activity (Nicolas-Alonso and Gomez-Gil 2012; Rao 2013). The hemodynamic response has an intrinsic onset delay of at least 1-3 s with respect to its associated electrical response from neural activity (Coyle, Ward and Markham 2007; Fazli, Mehnert, Steinbrink, Curio, Villringer, Müller and Blankertz 2012; Strait and Scheutz 2014b).

In case of fNIRS based BMIs, in order to better understand how particular architectures can enhance its performance, a better understanding of the intracranial infrared light path and the physiology-based systemic interferences on the superficial layers of the head is needed.

The fNIRS channel is composed by: a) a pair of infrared light source and light receptor, b) the light path between them. This light path is concave and as further the detector is placed from the source, deeper the light path penetrates into the head. The near infrared light travels into the skull, where it is reflected and absorbed by $\mathrm{HbO}$ and $\mathrm{HbR}$ (oxygenated and deoxygenated hemoglobin, respectively), whose concentration changes indicate activity in certain brain areas. Therefore, by measuring the reflected light, it is possible to estimate the concentration changes of $\mathrm{HbO}$ and $\mathrm{HbR}$ in a certain path between the emitter and the receptor. However, a the receptor also captures the light reflected by superficial layers of the head, e.g. scalp, skull, and part of the brain tissue itself, which do not represent brain activity, but systemic interferences or artifacts from cardiac activity, respiration, blood pressure 
variation and other underlying homeostatic processes (Ebihara et al. 2013; Gagnon et al. 2012; Matteau-Pelletier et al. 2009). 


\section{ANNEX A MATLAB SCRIPT: SSVEP TASK}

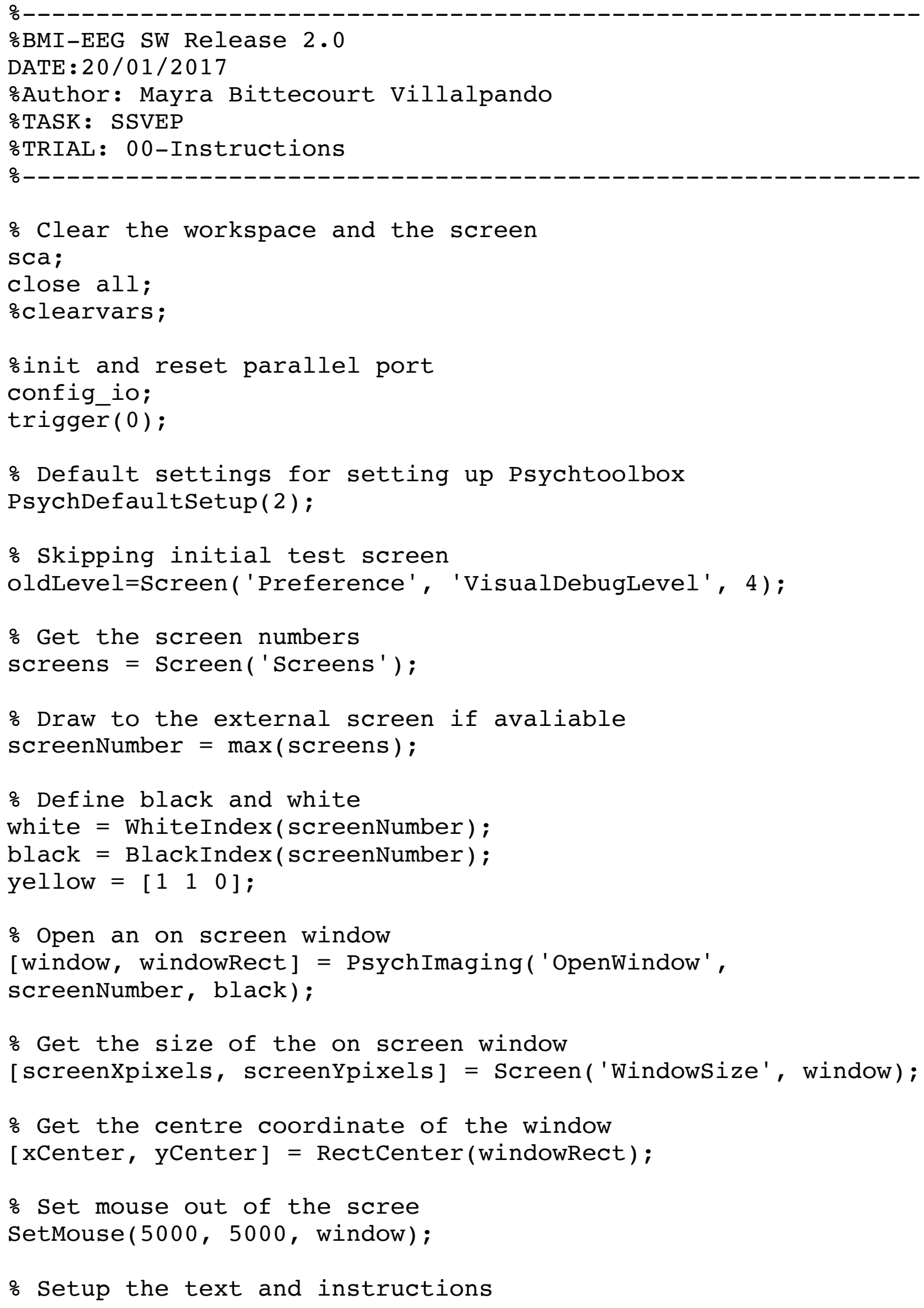




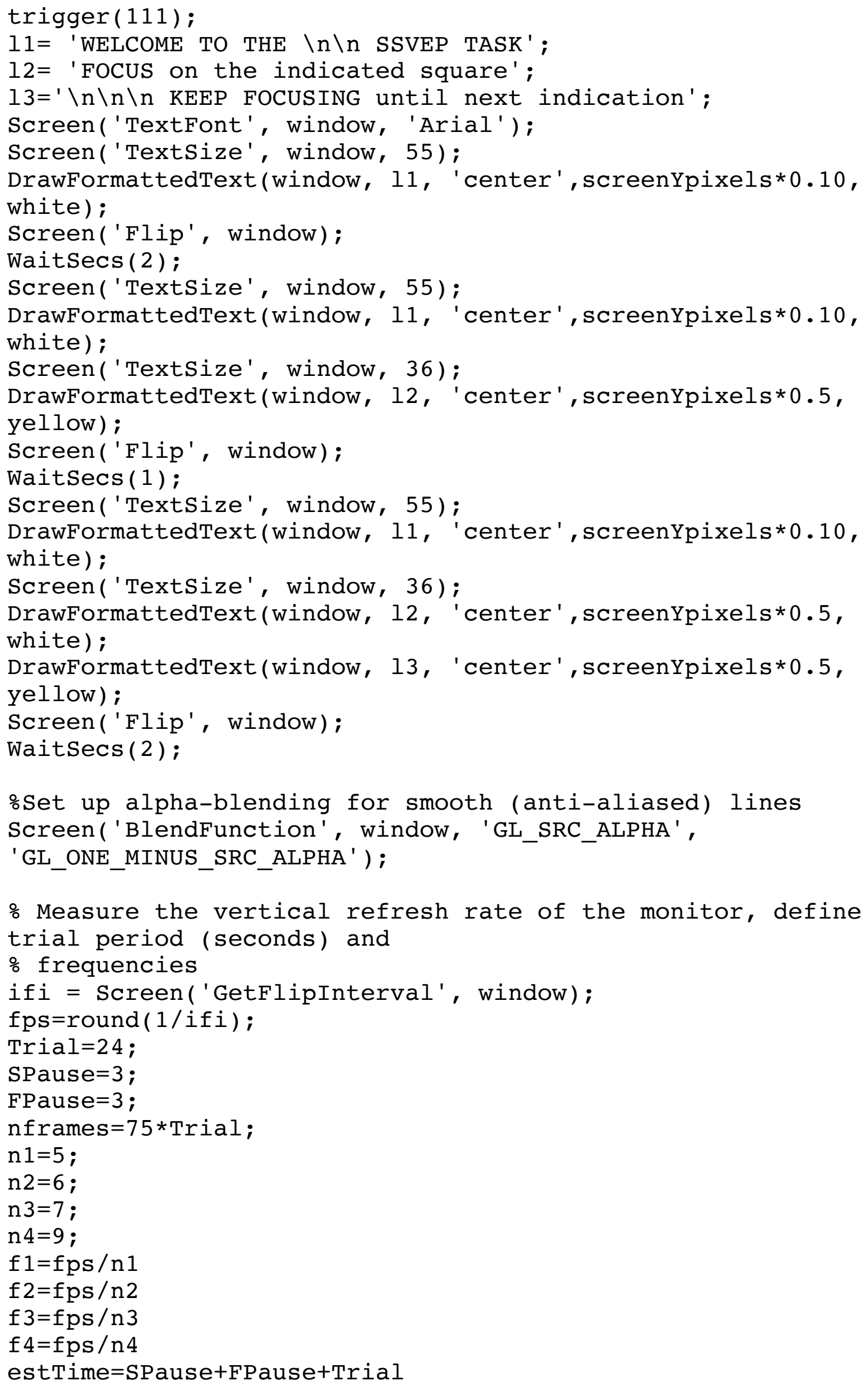


\% Use waitframes to flip at a rate that is different from monitor refresh rate. Waitframes: number of frames to wait before flipping to next frame.

waitframes $=1$;

$\%$ Screen $\mathrm{X}$ and $\mathrm{Y}$ positions of the 4 rectangles squarexpos $=[$ screenXpixels $* 0.25$ screenXpixels $* 0.75$ screenXpixels * 0.25 screenXpixels * 0.75$]$; squareYpos $=[$ screenYpixels $* 0.25$ screenYpixels $* 0.25$ screenYpixels * 0.75 screenYpixels * 0.75]; numsquares $=$ length (squareXpos);

\% Screen $\mathrm{X}$ and $\mathrm{Y}$ positions of the cues cuexpos = squarexpos; cueYpos = squareYpos;

\% Set the stimuli RGB color matrixes (Stimulus is white (1), interval is black (0))

At $=$ zeros ( 3 , nframes ) ;

for $i=1: n 1:$ nframes

for $j=1: 3$

At $(j, i)=1$;

end

At $(j, i+1)=1$;

end

Bt $=$ zeros ( 3 , nframes ) ;

for $i=1: n 2:$ nframes

for $j=1: 3$

$\operatorname{Bt}(j, i)=1$;

end

$\operatorname{Bt}(j, i+1)=1$;

end

Ct=zeros ( 3 , nframes ) ;

for $i=1: n 3:$ nframes

for $j=1: 3$

$\operatorname{Ct}(j, i)=1$;

end

$\operatorname{Ct}(j, i+1)=1$;

end

Dt $=$ zeros $(3$, nframes $)$;

for $i=1: n 4:$ nframes

for $j=1: 3$

$\operatorname{Dt}(j, i)=1$;

end

$\operatorname{Dt}(j, i+1)=1$;

end

\% Make a base Rect of X by $Y$ pixels

baseRect $=\left[\begin{array}{llll}0 & 0 & 185 & 185\end{array}\right]$; 
cueRect $=\left[\begin{array}{llll}0 & 0 & 200 & 200\end{array}\right]$;

\% Make the SQUARES and CUES coordinates

allRects $=\operatorname{nan}(4,4)$;

allcueRects $=\operatorname{nan}(4,4)$;

for $i=1$ : numsquares

allRects $(:, i)=$ CenterRectonPointd (baseRect, squareXpos(i), squareYpos(i));

allcueRects $(:, i)=$ CenterRectonPointd (cueRect, cuexpos(i), cueYpos(i));

end

$\%$ Retreive the maximum priority number and set max priority topPriorityLevel = MaxPriority (window);

Priority(topPriorityLevel);

\%Initial Pause

tStart $=$ GetSecs;

FixCross ();

Flip outside of the loop to get a time stamp

$\mathrm{vbl}=\operatorname{Screen}($ 'Flip', window);

trigger (255);

$\mathrm{vbl}=\mathrm{vbl}+\mathrm{SPause}$;

event $=1$;

: Run until end of frames

for $\mathrm{k}=1:$ nframes

$A=A t(:, k)$;

$\mathrm{B}=\mathrm{Bt}(:, \mathrm{k})$;

$\mathrm{C}=\mathrm{Ct}(:, \mathrm{k})$;

$\mathrm{D}=\mathrm{Dt}(:, \mathrm{k})$;

if $(k<$ nframes $/ 4)$

Screen('FrameRect', window, yellow, allcueRects $(:, 1)$, $10)$;

elseif(k>=nframes $/ 4$ \&\& $\mathrm{k}<$ nframes $/ 2$ )

Screen('FrameRect', window, yellow, allcueRects $(:, 2)$, $10)$;

elseif(k>=nframes $/ 2$ \&\& $k<$ nframes*3/4)

Screen('FrameRect', window, yellow, allcueRects $(:, 3)$, $10)$;

else

Screen('FrameRect', window, yellow, allcueRects $(:, 4)$, $10)$;

end

allColors $=\left[\begin{array}{llll}A & B & C & D\end{array}\right]$;

\% Draw the rect to the screen

Screen('FillRect', window, allColors, allRects);

:Flip to the screen

$\mathrm{vbl}=$ Screen ('Flip', window, $\mathrm{vbl}+0.5 * i f i)$;

if $(\mathrm{k}==1|| \mathrm{k}==\mathrm{nframes} / 4|| \mathrm{k}==\mathrm{nframes} / 2|| \mathrm{k}==$ nframes $* 3 / 4)$

trigger(event); 


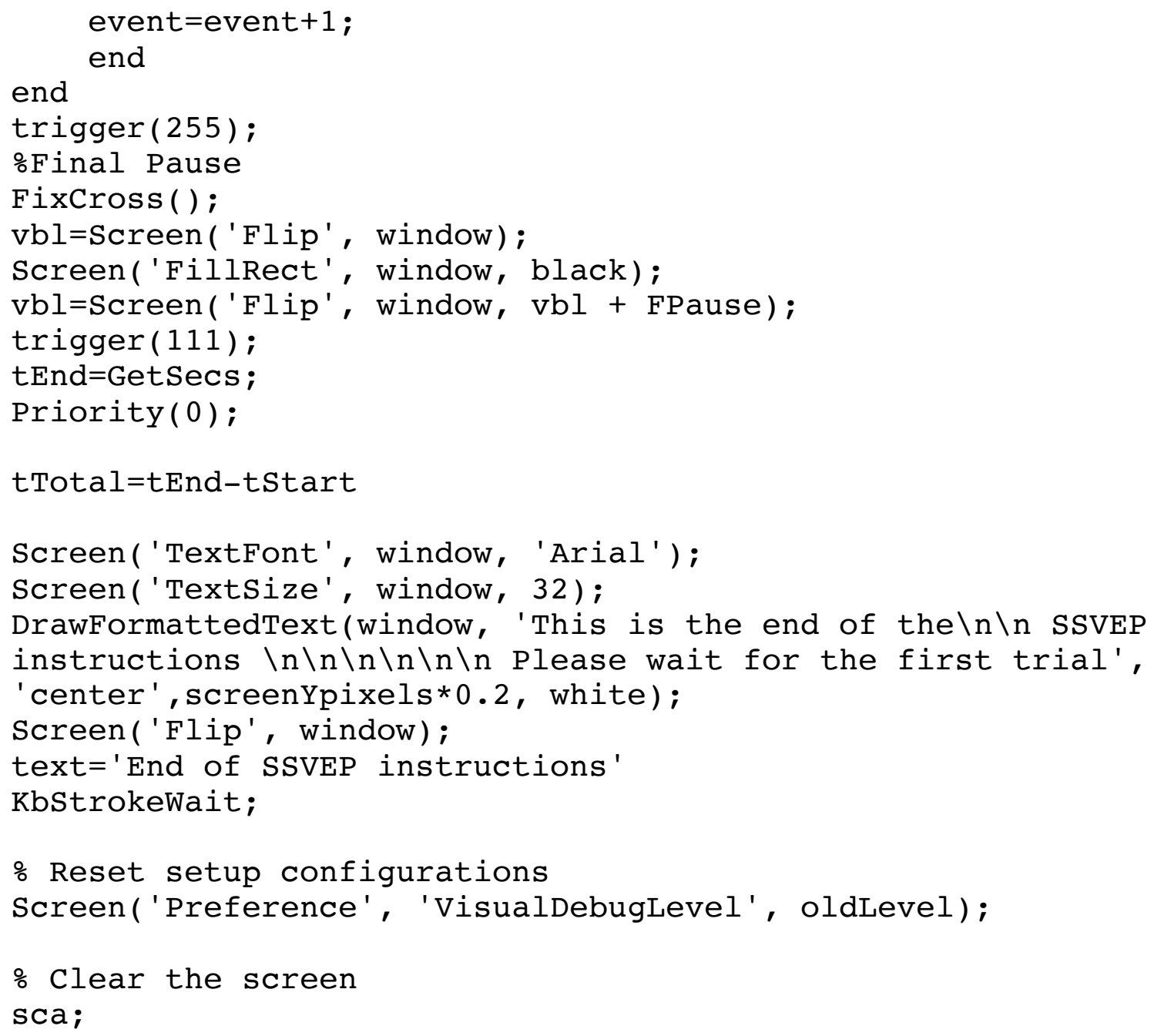




\section{REFERENCES}

ABDELNOUR, A. F. AND T. HUPPERT Real-time imaging of human brain function by near-infrared spectroscopy using an adaptive general linear model. Neuroimage, May 2009, 46(1), 133-143.

ABIBULLAEV, B. AND J. AN Classification of frontal cortex haemodynamic responses during cognitive tasks using wavelet transforms and machine learning algorithms. Medical Engineering and Physics, 2012, 34(10), 1394-1410.

ALLISON, B., T. LUTH, D. VALBUENA, A. TEYMOURIAN, et al. BCl demographics: how many (and what kinds of) people can use an SSVEP BCI? IEEE Trans Neural Syst Rehabil Eng, Apr 2010, 18(2), 107-116.

AMIRI, M., P. POULIOT, C. BONNÉRY, P. O. LECLERC, et al. An Exploration of the Effect of Hemodynamic Changes Due to Normal Aging on the fNIRS Response to Semantic Processing of Words. Front Neurol, 2014, 5, 249.

AQIL, M., K. S. HONG, M. Y. JEONG AND S. S. GE Cortical brain imaging by adaptive filtering of NIRS signals. Neurosci Lett, Apr 2012, 514(1), 35-41.

ARVANEH, M., C. GUAN, K. K. ANG AND C. QUEK Optimizing the channel selection and classification accuracy in EEG-based BCl. IEEE Trans Biomed Eng, Jun 2011, 58(6), 1865-1873.

BALCONI, M., E. GRIPPA AND M. E. VANUTELLI What hemodynamic (fNIRS), electrophysiological (EEG) and autonomic integrated measures can tell us about emotional processing. Brain Cogn, Apr 2015, 95, 67-76.

BAUERNFEIND, G., R. LEEB, S. C. WRIESSNEGGER AND G. PFURTSCHELLER Development, set-up and first results for a one-channel near-infrared spectroscopy system. Biomed Tech (Berl), Feb 2008, 53(1), 36-43.

BAUERNFEIND, G., S. C. WRIESSNEGGER, I. DALY AND G. R. MÜLLER-PUTZ Separating heart and brain: On the reduction of physiological noise from multichannel functional near-infrared spectroscopy (fNIRS) signals. Journal of Neural Engineering, 2014, 11(5).

BEAR, M. F., B. W. CONNORS AND M. A. PARADISO Neuroscience : exploring the brain. Edtion ed. Philadelphia: Wolters Kluwer, 2016. xlii, 975 pages p. ISBN 9780781778176

0781778174. 
BLOKLAND, Y., L. SPYROU, D. THIJSSEN, T. EIJSVOGELS, et al. Combined EEGfNIRS decoding of motor attempt and imagery for brain switch control: an offline study in patients with tetraplegia. IEEE Trans Neural Syst Rehabil Eng, Mar 2014, 22(2), 222-229.

BODEN, S., H. OBRIG, C. KÖHNCKE, H. BENAV, et al. The oxygenation response to functional stimulation: is there a physiological meaning to the lag between parameters? Neuroimage, May 2007, 36(1), 100-107.

BOGLER, C., J. MEHNERT, J. STEINBRINK AND J. D. HAYNES Decoding vigilance with NIRS. PLoS One, 2014, 9(7), e101729.

BRAINARD, D. H. The psychophysics toolbox. Spatial vision, 1997, 10, 433-436.

BRUNNER, C., N. BIRBAUMER, B. BLANKERTZ, C. GUGER, et al. BNCI Horizon 2020: towards a roadmap for the $\mathrm{BCl}$ community. Brain-Computer Interfaces, 2015, 2(1), 1-10.

BUZSAIKI, G. Rhythms of the brain. Edtion ed. Oxford: Oxford University Press, 2006. ISBN 9780195301069 (hbk.) : ${ }^{142.00}$

0195301064 (hbk.) : ${ }^{142.00 .}$

CHEN, C. H., M. S. HO, K. K. SHYU, K. C. HSU, et al. A noninvasive brain computer interface using visually-induced near-infrared spectroscopy responses. Neuroscience Letters, 2014, 580, 22-26.

CHEN, L. C., P. SANDMANN, J. D. THORNE, C. S. HERRMANN, et al. Association of Concurrent fNIRS and EEG Signatures in Response to Auditory and Visual Stimuli. Brain Topogr, Jan 2015.

COMBAZ, A. AND M. M. VAN HULLE Simultaneous detection of P300 and steadystate visually evoked potentials for hybrid brain-computer interface. PLoS One, 2015, 10(3), e0121481.

COYLE, S., T. WARD AND C. MARKHAM Physiological noise in near-infrared spectroscopy: implications for optical brain computer interfacing. Conf Proc IEEE Eng Med Biol Soc, 2004, 6, 4540-4543.

COYLE, S. M., T. E. WARD AND C. M. MARKHAM Brain-computer interface using a simplified functional near-infrared spectroscopy system. J Neural Eng, Sep 2007, 4(3), 219-226. 
CUTINI, S. AND S. BRIGADOI Unleashing the future potential of functional nearinfrared spectroscopy in brain sciences. Journal of Neuroscience Methods, 2014, 232, $152-156$.

DONCHIN, E., K. M. SPENCER AND R. WIJESINGHE The mental prosthesis: assessing the speed of a P300-based brain-computer interface. IEEE Trans Rehabil Eng, Jun 2000, 8(2), 174-179.

DURANTIN, G., S. SCANNELLA, T. GATEAU, A. DELORME, et al. Moving Average Convergence Divergence filter preprocessing for real-time event-related peak activity onset detection : application to fNIRS signals. Conf Proc IEEE Eng Med Biol Soc, 2014, 2014, 2107-2110.

EBIHARA, A., Y. TANAKA, T. KONNO, S. KAWASAKI, et al. Detection of cerebral ischemia using the power spectrum of the pulse wave measured by near-infrared spectroscopy. J Biomed Opt, Oct 2013, 18(10), 106001.

FABIANI, M., B. A. GORDON, E. L. MACLIN, M. A. PEARSON, et al. Neurovascular coupling in normal aging: a combined optical, ERP and fMRI study. Neuroimage, Jan 2014, 85 Pt 1, 592-607.

FARWELL, L. A. AND E. DONCHIN Talking off the top of your head: toward a mental prosthesis utilizing event-related brain potentials. Electroencephalogr Clin Neurophysiol, Dec 1988, 70(6), 510-523.

FAZLI, S., J. MEHNERT, J. STEINBRINK, G. CURIO, et al. Enhanced performance by a hybrid NIRS-EEG brain computer interface. Neuroimage, Jan 2012, 59(1), 519529.

FUJIWARA, N., K. SAKATANI, Y. KATAYAMA, Y. MURATA, et al. Evoked-cerebral blood oxygenation changes in false-negative activations in BOLD contrast functional MRI of patients with brain tumors. Neuroimage, Apr 2004, 21(4), 1464-1471.

FUNANE, T., H. ATSUMORI, M. KIGUCHI, Y. TANIKAWA, et al. Dynamic phantom with two stage-driven absorbers for mimicking hemoglobin changes in superficial and deep tissues. J Biomed Opt, Apr 2012, 17(4), 047001.

GAGNON, L., R. J. COOPER, M. A. YÜCEL, K. L. PERDUE, et al. Short separation channel location impacts the performance of short channel regression in NIRS. Neuroimage, Feb 2012, 59(3), 2518-2528. 
GAGNON, L., K. PERDUE, D. N. GREVE, D. GOLDENHOLZ, et al. Improved recovery of the hemodynamic response in diffuse optical imaging using short optode separations and state-space modeling. Neuroimage, Jun 2011, 56(3), 1362-1371.

GAO, X., D. XU, M. CHENG AND S. GAO A BCl-based environmental controller for the motion-disabled. IEEE Trans Neural Syst Rehabil Eng, Jun 2003, 11(2), 137-140.

GEMBLER, F., P. STAWICKI AND I. VOLOSYAK Autonomous Parameter Adjustment for SSVEP-Based BCls with a Novel BCI Wizard. Front Neurosci, 2015, 9,474 .

GLASSER, M. F., T. S. COALSON, E. C. ROBINSON, C. D. HACKER, et al. A multimodal parcellation of human cerebral cortex. Nature, 2016.

HALDER, S., A. PINEGgER, I. KÄTHNER, S. C. WRIESSNEGGER, et al. Braincontrolled applications using dynamic P300 speller matrices. Artif Intell Med, Jan 2015, 63(1), 7-17.

HAMEDI, M., S. H. SALLEH AND A. M. NOOR Electroencephalographic Motor Imagery Brain Connectivity Analysis for BCl: A Review. Neural Comput, Jun 2016, 28(6), 999-1041.

HEILBRONNER, U. AND T. F. MÜNTE Rapid event-related near-infrared spectroscopy detects age-related qualitative changes in the neural correlates of response inhibition. Neuroimage, Jan 2013, 65, 408-415.

JIN, J., B. Z. ALLISON, T. KAUFMANN, A. KÜBLER, et al. The changing face of P300 BCls: a comparison of stimulus changes in a P300 BCl involving faces, emotion, and movement. PLoS One, 2012, 7(11), e49688.

JOHNSON, R. On the neural generators of the P300 component of the event-related potential. Psychophysiology, Jan 1993, 30(1), 90-97.

KAHLAOUI, K., G. DI SANTE, J. BARBEAU, M. MAHEUX, et al. Contribution of NIRS to the study of prefrontal cortex for verbal fluency in aging. Brain Lang, May 2012, 121(2), 164-173.

KAISER, V., G. BAUERNFEIND, A. KREILINGER, T. KAUFMANN, et al. Cortical effects of user training in a motor imagery based brain-computer interface measured by fNIRS and EEG. Neurolmage, 2014, 85, 432-444. 
KAMEYAMA, M., M. FUKUDA, T. UEHARA AND M. MIKUNI Sex and age dependencies of cerebral blood volume changes during cognitive activation: a multichannel near-infrared spectroscopy study. Neuroimage, Aug 2004, 22(4), 17151721.

KAUFMANN, T., S. M. SCHULZ, C. GRÜNZINGER AND A. KÜBLER Flashing characters with famous faces improves ERP-based brain-computer interface performance. J Neural Eng, Oct 2011, 8(5), 056016.

KAUFMANN, T., S. M. SCHULZ, A. KÖBLITZ, G. RENNER, et al. Face stimuli effectively prevent brain-computer interface inefficiency in patients with neurodegenerative disease. Clin Neurophysiol, May 2013, 124(5), 893-900.

KHAN, M. J., M. J. HONG AND K. S. HONG Decoding of four movement directions using hybrid NIRS-EEG brain-computer interface. Front Hum Neurosci, 2014, 8, 244.

KLEINER, M., D. BRAINARD, D. PELLI, A. INGLING, et al. What's new in Psychtoolbox-3. Perception, 2007, 36(14), 1.

KOH, P. H., D. E. GLASER, G. FLANDIN, S. KIEBEL, et al. Functional optical signal analysis: a software tool for near-infrared spectroscopy data processing incorporating statistical parametric mapping. J Biomed Opt, 2007 Nov-Dec 2007, 12(6), 064010.

KUŚ, R., A. DUSZYK, P. MILANOWSKI, M. ŁABĘCKI, et al. On the quantification of SSVEP frequency responses in human EEG in realistic $\mathrm{BCl}$ conditions. PLoS One, 2013, 8(10), e77536.

LUU, S. AND T. CHAU Decoding subjective preference from single-trial near-infrared spectroscopy signals. J Neural Eng, Feb 2009, 6(1), 016003.

MACHADO, A., J. M. LINA, J. TREMBLAY, M. LASSONDE, et al. Detection of hemodynamic responses to epileptic activity using simultaneous ElectroEncephaloGraphy (EEG)/Near Infra Red Spectroscopy (NIRS) acquisitions. Neuroimage, May 2011, 56(1), 114-125.

MATTEAU-PELLETIER, C., M. DEHAES, F. LESAGE AND J. M. LINA $1 / \mathrm{f}$ noise in diffuse optical imaging and wavelet-based response estimation. IEEE Trans Med Imaging, Mar 2009, 28(3), 415-422.

MATTHEWS, F., B. A. PEARLMUTTER, T. E. WARD, C. SORAGHAN, et al. Hemodynamics for brain-computer interfaces. IEEE Signal Processing Magazine, 2008, 25(1), 87-94. 
MCFARLAND, D. J. AND J. R. WOLPAW Brain-Computer Interfaces for Communication and Control. Commun ACM, 2011, 54(5), 60-66.

MERTENS, R. AND J. POLICH P300 from a single-stimulus paradigm: passive versus active tasks and stimulus modality. Electroencephalogr Clin Neurophysiol, Nov 1997, 104(6), 488-497.

MIDDENDORF, M., G. MCMILLAN, G. CALHOUN AND K. S. JONES Braincomputer interfaces based on the steady-state visual-evoked response. IEEE Trans Rehabil Eng, Jun 2000, 8(2), 211-214.

MILLER, K. J., G. SCHALK, E. E. FETZ, M. DEN NIJS, et al. Cortical activity during motor execution, motor imagery, and imagery-based online feedback. Proc Natl Acad Sci U S A, Mar 2010, 107(9), 4430-4435.

MORIOKA, H., A. KANEMURA, S. MORIMOTO, T. YOSHIOKA, et al. Decoding spatial attention by using cortical currents estimated from electroencephalography with near-infrared spectroscopy prior information. Neuroimage, Apr 2014, 90, 128139.

MÜLLER, K.-R., M. TANGERMANN, G. DORNHEGE, M. KRAULEDAT, et al. Machine learning for real-time single-trial EEG-analysis: From brain-computer interfacing to mental state monitoring. Brain-Computer Interfaces (BCls), 2008, 167(1), 82-90.

MÜLLER-PUTZ, G. R. AND G. PFURTSCHELLER Control of an electrical prosthesis with an SSVEP-based BCl. IEEE Trans Biomed Eng, Jan 2008, 55(1), 361-364.

NAKAI, T., E. BAGARINAO, Y. TANAKA, K. MATSUO, et al. Ontology for FMRI as a biomedical informatics method. Magn Reson Med Sci, 2008, 7(3), 141-155.

NAKAO, T., T. MATSUMOTO, D. SHIMIZU, M. MORITA, et al. Resting state lowfrequency fluctuations in prefrontal cortex reflect degrees of harm avoidance and novelty seeking: an exploratory NIRS study. Front Syst Neurosci, 2013, 7, 115.

NASEER, N. AND K. S. HONG Classification of functional near-infrared spectroscopy signals corresponding to the right- and left-wrist motor imagery for development of a brain-computer interface. Neuroscience Letters, 2013, 553, 84-89.

NEUPER, C., R. SCHERER, S. WRIESSNEGGER AND G. PFURTSCHELLER Motor imagery and action observation: modulation of sensorimotor brain rhythms during mental control of a brain-computer interface. Clin Neurophysiol, Feb 2009, 120(2), 239-247. 
NEUPER, C., A. SCHLÖGL AND G. PFURTSCHELLER Enhancement of left-right sensorimotor EEG differences during feedback-regulated motor imagery. J Clin Neurophysiol, Jul 1999, 16(4), 373-382.

NICOLAS-ALONSO, L. F. AND J. GOMEZ-GIL Brain computer interfaces, a review. Sensors (Basel), 2012, 12(2), 1211-1279.

OKAMOTO, M., H. DAN, K. SAKAMOTO, K. TAKEO, et al. Three-dimensional probabilistic anatomical cranio-cerebral correlation via the international $10-20$ system oriented for transcranial functional brain mapping. Neuroimage, Jan 2004, 21(1), 99111.

PASTOR, M. A., J. ARTIEDA, J. ARBIZU, M. VALENCIA, et al. Human cerebral activation during steady-state visual-evoked responses. J Neurosci, Dec 2003, 23(37), 11621-11627.

PELLI, D. G. The VideoToolbox software for visual psychophysics: Transforming numbers into movies. Spatial vision, 1997, 10(4), 437-442.

PFURTSCHELLER, G., B. Z. ALLISON, C. BRUNNER, G. BAUERNFEIND, et al. The hybrid BCl. Front Neurosci, 2010, 4, 30.

PFURTSCHELLER, G., C. NEUPER, C. GUGER, W. HARKAM, et al. Current trends in Graz Brain-Computer Interface $(\mathrm{BCl})$ research. IEEE Trans Rehabil Eng, Jun 2000, 8(2), 216-219.

PFURTSCHELLER, G., C. NEUPER, G. R. MÜLLER, B. OBERMAIER, et al. Graz$\mathrm{BCl}$ : state of the art and clinical applications. IEEE Trans Neural Syst Rehabil Eng, Jun 2003, 11(2), 177-180.

PFURTSCHELLER, G., C. NEUPER, A. SCHLÖGL AND K. LUGGER Separability of EEG signals recorded during right and left motor imagery using adaptive autoregressive parameters. IEEE Trans Rehabil Eng, Sep 1998, 6(3), 316-325.

PIERCE, J. R. An Introduction to Information Theory: Symbols. Signals and Noise E, 1980, 2.

POLICH, J. Updating P300: an integrative theory of P3a and P3b. Clin Neurophysiol, Oct 2007, 118(10), 2128-2148. 
POWER, S. D., T. H. FALK AND T. CHAU Classification of prefrontal activity due to mental arithmetic and music imagery using hidden Markov models and frequency domain near-infrared spectroscopy. J Neural Eng, Apr 2010, 7(2), 26002.

POWER, S. D., A. KUSHKI AND T. CHAU Automatic single-trial discrimination of mental arithmetic, mental singing and the no-control state from prefrontal activity: toward a three-state NIRS-BCl. BMC Res Notes, 2012, 5, 141.

PURVES, D. Neuroscience. Edtion ed. Sunderland, Mass.: Sinauer Associates, Publishers, 2004. ISBN 0878937250 (casebound : alk. paper) : ${ }^{141.99 .}$

PUTZE, F., S. HESSLINGER, C. Y. TSE, Y. HUANG, et al. Hybrid fNIRS-EEG based classification of auditory and visual perception processes. Frontiers in Neuroscience, 2014, 8(OCT).

RAO, R. P. N. Brain-Computer Interfacing. Edtion ed.: Cambridge University Press, 2013. ISBN 9780521769419.

RAVDEN, D. AND J. POLICH On P300 measurement stability: habituation, intra-trial block variation, and ultradian rhythms. Biol Psychol, Oct 1999, 51(1), 59-76.

ROWLING, J. K. Harry Potter and the chamber of secrets. Edtion ed. London: Bloomsbury, 1999. ISBN 9780747538486 (pbk) : ${ }^{16} .99$.

SANEI, S. AND J. CHAMBERS EEG signal processing. Edtion ed. Hoboken, N.J.: Wiley ; Chichester : John Wiley [distributor], 2007. ISBN 9780470025819 (cased) : ${ }^{1} 65.00$

0470025816 (cased) : ${ }^{1} 65.00$.

SCHLÖGL, A., F. LEE, H. BISCHOF AND G. PFURTSCHELLER Characterization of four-class motor imagery EEG data for the BCl-competition 2005. J Neural Eng, Dec 2005, 2(4), L14-22.

SCHOLKMANN, F., S. KLEISER, A. J. METZ, R. ZIMMERMANN, et al. A review on continuous wave functional near-infrared spectroscopy and imaging instrumentation and methodology. Neuroimage, Jan 2014, 85 Pt 1, 6-27.

SCHWARTZ, A. B., X. T. CUI, D. J. WEBER AND D. W. MORAN Brain-controlled interfaces: movement restoration with neural prosthetics. Neuron, Oct 2006, 52(1), 205-220. 
SELB, J., T. M. OGDEN, J. DUBB, Q. FANG, et al. Comparison of a layered slab and an atlas head model for Monte Carlo fitting of time-domain near-infrared spectroscopy data of the adult head. J Biomed Opt, Jan 2014, 19(1), 16010.

SHAN, H., H. XU, S. ZHU AND B. HE A novel channel selection method for optimal classification in different motor imagery BCl paradigms. Biomed Eng Online, 2015, $14,93$.

SINGH, A. K. AND I. DAN Exploring the false discovery rate in multichannel NIRS. Neuroimage, Nov 2006, 33(2), 542-549.

SITARAM, R., A. CARIA AND N. BIRBAUMER Hemodynamic brain-computer interfaces for communication and rehabilitation. Neural Netw, Nov 2009, 22(9), 13201328.

SITARAM, R., H. ZHANG, C. GUAN, M. THULASIDAS, et al. Temporal classification of multichannel near-infrared spectroscopy signals of motor imagery for developing a brain-computer interface. Neuroimage, Feb 2007, 34(4), 1416-1427.

STRAIT, M. AND M. SCHEUTZ What we can and cannot (yet) do with functional near infrared spectroscopy. Frontiers in Neuroscience, 2014a, (8 MAY).

STRAIT, M. AND M. SCHEUTZ What we can and cannot (yet) do with functional near infrared spectroscopy. Front Neurosci, 2014b, 8, 117.

STUBAN, N., M. NIWAYAMA AND H. SANTHA Phantom with pulsatile arteries to investigate the influence of blood vessel depth on pulse oximeter signal strength. Sensors (Basel), 2012, 12(1), 895-904.

SUN, B., L. ZHANG, H. GONG, J. SUN, et al. Detection of optical neuronal signals in the visual cortex using continuous wave near-infrared spectroscopy. Neuroimage, Feb 2014, 87, 190-198.

TACHIBANA, A., J. A. NOAH, S. BRONNER, Y. ONO, et al. Parietal and temporal activity during a multimodal dance video game: an fNIRS study. Neurosci Lett, Oct 2011, 503(2), 125-130.

TAI, K. AND T. CHAU Single-trial classification of NIRS signals during emotional induction tasks: towards a corporeal machine interface. J Neuroeng Rehabil, 2009, $6,39$. 
TAKEUCHI, M., E. HORI, K. TAKAMOTO, A. H. TRAN, et al. Brain cortical mapping by simultaneous recording of functional near infrared spectroscopy and electroencephalograms from the whole brain during right median nerve stimulation. Brain Topogr, Nov 2009, 22(3), 197-214.

TANAKA, H. AND T. KATURA Classification of change detection and change blindness from near-infrared spectroscopy signals. J Biomed Opt, Aug 2011, 16(8), 087001.

TEHOVNIK, E. J. AND L. L. CHEN Brain control and information transfer. Exp Brain Res, Dec 2015, 233(12), 3335-3347.

TEHOVNIK, E. J., L. C. WOODS AND W. M. SLOCUM Transfer of information by BMI. Neuroscience, 2013, 255, 134-146.

THOMPSON, D. E., L. R. QUITADAMO, L. MAINARDI, K. U. LAGHARI, et al. Performance measurement for brain-computer or brain-machine interfaces: a tutorial. J Neural Eng, Jun 2014, 11(3), 035001.

TIAN, F. AND H. LIU Depth-compensated diffuse optical tomography enhanced by general linear model analysis and an anatomical atlas of human head. Neuroimage, Jan 2014, 85 Pt 1, 166-180.

TOMITA, Y., F. B. VIALATTE, G. DREYFUS, Y. MITSUKURA, et al. Bimodal BCI using simultaneously NIRS and EEG. IEEE Transactions on Biomedical Engineering, 2014, 61(4), 1274-1284.

TONG, Y. AND B. D. FREDERICK Time lag dependent multimodal processing of concurrent fMRI and near-infrared spectroscopy (NIRS) data suggests a global circulatory origin for low-frequency oscillation signals in human brain. Neuroimage, Nov 2010, 53(2), 553-564.

TONG, Y., L. M. HOCKE, S. C. LICATA AND B. FREDERICK Low-frequency oscillations measured in the periphery with near-infrared spectroscopy are strongly correlated with blood oxygen level-dependent functional magnetic resonance imaging signals. J Biomed Opt, Oct 2012, 17(10), 106004.

TONG, Y., K. P. LINDSEY AND B. DEB FREDERICK Partitioning of physiological noise signals in the brain with concurrent near-infrared spectroscopy and fMRI. J Cereb Blood Flow Metab, Dec 2011, 31(12), 2352-2362. 
TOWNSEND, G., B. K. LAPALLO, C. B. BOULAY, D. J. KRUSIENSKI, et al. A novel P300-based brain-computer interface stimulus presentation paradigm: moving beyond rows and columns. Clin Neurophysiol, Jul 2010, 121(7), 1109-1120.

TOWNSEND, G. AND V. PLATSKO Pushing the P300-based brain-computer interface beyond $100 \mathrm{bpm}$ : extending performance guided constraints into the temporal domain. J Neural Eng, Apr 2016, 13(2), 026024.

UEHARA, T., M. FUKUDA, M. SUDA, M. ITO, et al. Cerebral blood volume changes in patients with eating disorders during word fluency: a preliminary study using multichannel near infrared spectroscopy. Eat Weight Disord, Dec 2007, 12(4), 183-190.

VIDAURRE, C. AND B. BLANKERTZ Towards a cure for BCI illiteracy. Brain Topogr, Jun 2010, 23(2), 194-198.

VOLOSYAK, I. SSVEP-based Bremen-BCI interface--boosting information transfer rates. J Neural Eng, Jun 2011, 8(3), 036020.

WANG, M., I. DALY, B. Z. ALLISON, J. JIN, et al. A new hybrid BCI paradigm based on P300 and SSVEP. J Neurosci Methods, Apr 2015, 244, 16-25.

WILCOX, T., J. STUBBS, A. HIRSHKOWITZ AND D. A. BOAS Functional activation of the infant cortex during object processing. Neuroimage, Sep 2012, 62(3), 18331840.

WOLPAW, J. R., N. BIRBAUMER, W. J. HEETDERKS, D. J. MCFARLAND, et al. Brain-computer interface technology: a review of the first international meeting. IEEE Trans Rehabil Eng, Jun 2000, 8(2), 164-173.

WOLPAW, J. R., N. BIRBAUMER, D. J. MCFARLAND, G. PFURTSCHELLER, et al. Brain-computer interfaces for communication and control. Clin Neurophysiol, Jun 2002, 113(6), 767-791.

WOLPAW, J. R., D. J. MCFARLAND, T. M. VAUGHAN AND G. SCHALK The Wadsworth Center brain-computer interface $(\mathrm{BCl})$ research and development program. IEEE Trans Neural Syst Rehabil Eng, Jun 2003, 11(2), 204-207.

WOLPAW, J. R. AND E. W. WOLPAW Brain-computer interfaces : principles and practice. Edtion ed. Oxford ; New York: Oxford University Press, 2012. ISBN 9780195388855 (hbk.) : No price

0195388852 (hbk.) : No price. 
YE, J. C., S. TAK, K. E. JANG, J. JUNG, et al. NIRS-SPM: statistical parametric mapping for near-infrared spectroscopy. Neuroimage, Jan 2009, 44(2), 428-447.

YIN, X., B. XU, C. JIANG, Y. FU, et al. A hybrid BCl based on EEG and fNIRS signals improves the performance of decoding motor imagery of both force and speed of hand clenching. Journal of Neural Engineering, 2015, 12(3).

YUAN, P., X. GAO, B. ALLISON, Y. WANG, et al. A study of the existing problems of estimating the information transfer rate in online brain-computer interfaces. J Neural Eng, Apr 2013, 10(2), 026014.

Z. ALLISON, B., J. JIN, Y. ZHANG AND X. WANG A four-choice hybrid P300/SSVEP $\mathrm{BCl}$ for improved accuracy. Brain-Computer Interfaces, 2014, 1(1), 17-26.

ZHANG, Q., G. E. STRANGMAN AND G. GANIS Adaptive filtering to reduce global interference in non-invasive NIRS measures of brain activation: how well and when does it work? Neuroimage, Apr 2009, 45(3), 788-794.

ZHANG, Y. AND S. M. CHASE A stabilized dual Kalman filter for adaptive tracking of brain-computer interface decoding parameters. Conf Proc IEEE Eng Med Biol Soc, 2013, 2013, 7100-7103. 